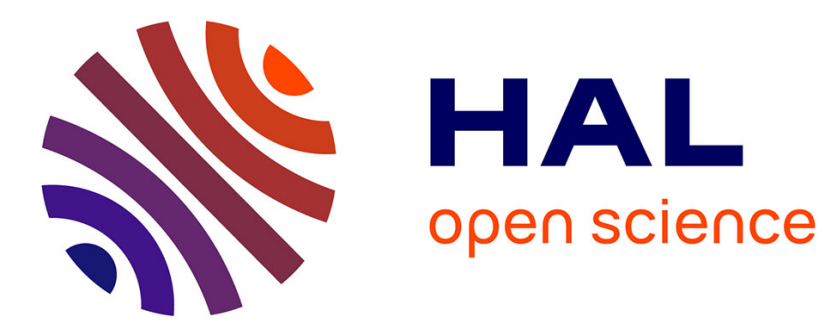

\title{
L'anthropologie démographique
}

\author{
Daniel Bley, Gilles Boëtsch
}

\section{To cite this version:}

Daniel Bley, Gilles Boëtsch. L'anthropologie démographique. PUF, 3441, 127 p., 1999, Collection

Que Sais-je, 2130496441. hal-01986867

\section{HAL Id: hal-01986867 \\ https://hal.science/hal-01986867}

Submitted on 13 Feb 2019

HAL is a multi-disciplinary open access archive for the deposit and dissemination of scientific research documents, whether they are published or not. The documents may come from teaching and research institutions in France or abroad, or from public or private research centers.
L'archive ouverte pluridisciplinaire HAL, est destinée au dépôt et à la diffusion de documents scientifiques de niveau recherche, publiés ou non, émanant des établissements d'enseignement et de recherche français ou étrangers, des laboratoires publics ou privés. 


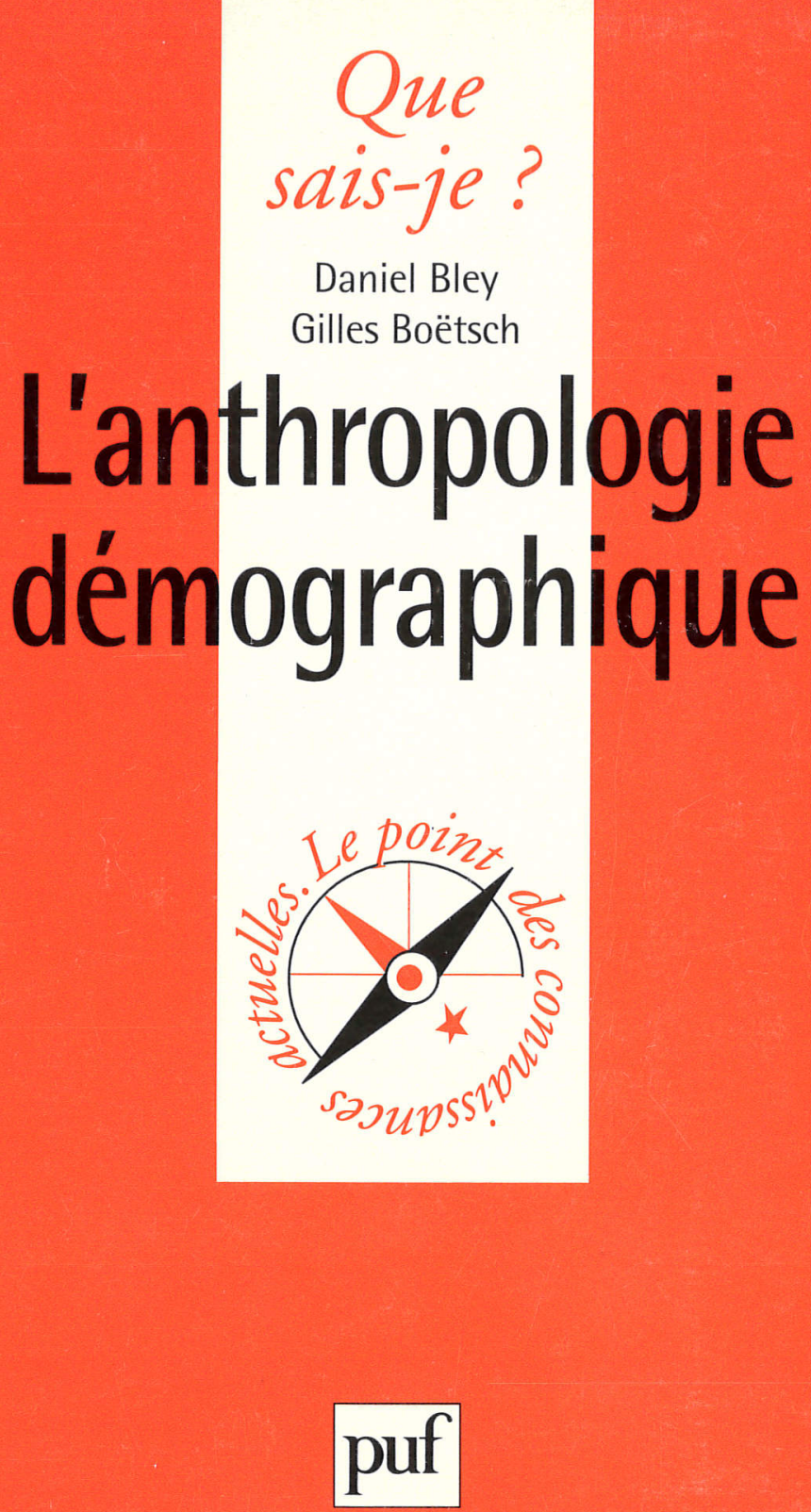


Q UE S A I S - J E ?

\title{
L'anthropologie démographique
}

\author{
DANIEL BLEY \\ Chargé de recherche au CNRS \\ GILLES BOETSCH \\ Directeur de recherche au CNRs
}

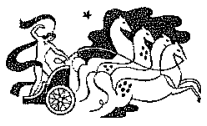


ISBN $213049644 \mathrm{x}$

Dépôt légal $-1^{\text {re }}$ édition : 1999 , mars

(C) Presses Universitaires de France, 1999

108, boulevard Saint-Germain, 75006 Paris 


\section{INTRODUCTION}

L'une des premières synthèses des travaux réalisés en anthropologie démographique a été publiée en 1986 par Nancy Howell. Intitulée Demographic Anthropology et comportant une bibliographie de 251 références, elle prend essentiellement en compte les recherches effectuées depuis 1970 et s'intéresse à la fois aux méthodes et aux principaux travaux théoriques de l'anthropologie démographique effectués sur de petites populations. L'auteur montre que les relations entre la démographie et l'anthropologie étaient auparavant presque inexistantes, les anthropologues étant le plus souvent rebutés par le caractère quantifié des études démographiques et les démographes totalement étrangers aux problématiques anthropologiques.

Cette situation a considérablement évolué en l'espace de deux décennies. D'une part, les démographes eux-mêmes se sont aperçus des limites des grandes enquêtes et de la qualité des données recueillies. Ces interrogations leur ont permis d'accorder alors plus d'importance aux questions soulevées par les autres sciences sociales et en particulier aux travaux plus documentés des anthropologues. D'autre part, les nouvelles techniques démographiques de mesure de fécondité et de mortalité ont été appliquées par les anthropologues sur les petites populations qu'ils avaient l'habitude d'étudier.

Les anthropologues ne se contentèrent pas de décrire les principaux paramètres démographiques, ils les intégrèrent dans leurs analyses pour mieux appré- 
cier les processus rendant compte de l'évolution biologique en élaborant par exemple les concepts d'effectif utile, du niveau de consanguinité, ou encore de carrying capacity. Les anthropologues développèrent aussi des méthodes originales et la multiplication de programmes informatisés pour la reconstruction de généalogies en est un bon exemple. Les travaux furent nombreux et cherchaient tous à comprendre la structure biodémographique de petites populations actuelles ou historiques, souvent isolées (rurales, insulaires, de montagne) ou de groupes de chasseurs-cueilleurs.

L'anthropologie démographique est donc un domaine de recherche aujourd'hui très actif qui se trouve confronté aux changements continuels qui affectent notre monde, notamment aux conséquences de la croissance démographique ou des mouvements internationaux de population. En utilisant les concepts et méthodes de la démographie, de la biologie des populations et de l'anthropologie culturelle et sociale, l'anthropologie démographique peut certainement contribuer à décrire et analyser de tels phénomènes. Certains travaux concernent l'organisation sociale des primates ou sont sécants avec l'archéologie ou la paléontologie et d'autres s'intéressent toujours aux chasseurs cueilleurs, aux populations rurales ou récemment aux populations en mouvement. La plupart des études se placent dans une perspective générale qui prend appui sur la théorie malthusienne, les anthropologues se focalisant plus particulièrement sur les composantes socioéconomiques et culturelles de la gestion des ressources et de la reproduction du groupe. Les études sur les populations agricoles, en montrant les relations existant entre économie, parenté, structures familiales et reproduction du groupe, illustrent bien cette tendance.

Dans cet ouvrage, une première partie présentera les principaux concepts et méthodes intervenant en 
anthropologie démographique. Nous aborderons successivement les notions d'évolution, de population, d'adaptation, de parenté pour ce qui concerne l'anthropologie. Puis nous traiterons de la question de la relation population/ressource, de la transition démographique et des méthodes d'analyse de la démographie. Enfin, nous présenterons les concepts indispensables pour nos analyses qui appartiennent au domaine de la génétique des populations.

Dans une deuxième partie, nous décrirons en quoi l'anthropologie démographique est un champ de recherche spécifique, en expliquant l'importance de la famille à la fois comme cadre et objet d'étude, le rôle $\mathrm{du}$ concept de génération, et celui de reproduction. Puis, nous présenterons les méthodes et analyses employées par l'anthropologie démographique.

Enfin dans une troisième partie, nous prendrons quelques exemples, ceux des populations de chasseurs-cueilleurs et des populations rurales des pays en développement et des pays industrialisés. 


\section{Chapitre I}

\section{CONCEPTS ET MÉTHODES}

La démographie, l'anthropologie biologique et la génétique des populations ont en commun de s'intéresser aux changements qui affectent les populations humaines tant sur les plans diachronique que synchronique. Mais les problématiques de ces trois disciplines demeurent différentes: la démographie décrit l'état des populations, la génétique des populations analyse les modifications des pools géniques alors que l'anthropologie biologique tente de suivre l'histoire biologique des hominidés et d'en apprécier les conséquences sur leur évolution.

\section{I. - Les concepts anthropologiques}

L'apparition de nouveaux concepts issus de la génétique a fait évoluer l'anthropologie durant la seconde moitié du $\mathrm{XX}^{\mathrm{c}}$ siècle. Des concepts comme celui de « gène » ou de «population » seront de première importance dans le développement d'une anthropologie biologique moderne (dite encore «populationnelle ») en permettant de dépasser les modèles explicatifs d'une anthropologie physique qui s'appuyait auparavant sur des concepts typologistes. L'anthropologie biologique s'inscrit désormais à l'interface entre la biologie et les sciences sociales. On n'étudie plus l' "homme » en soi, mais une population précise vivant dans des conditions environne- 
mentales spécifiques. De plus, la pratique scientifique de cette nouvelle anthropologie intègre l'approche quantitative. On insiste maintenant sur les variations individuelles existant à l'intérieur d'une même population et les différences observables sont alors attribuées à des phénomènes d'ordre adaptatif ou sélectif, puisque le génome est présupposé commun.

1. L'évolution, l'adaptation. - Le concept d'évolution forme un des paradigmes de la pensée biologique moderne. Il a été introduit par Lamarck (1809) et développé par Darwin (1859). Comme les autres organismes vivants, l'être humain se reproduit et évolue. En impliquant le croisement des lignées, la reproduction sexuelle contribue à maintenir la diversité biologique. Les processus qui font évoluer les populations sont la sélection et les mutations génétiques. Ces dernières provoquent rarement des changements spectaculaires et elles sont généralement très discrètes. On constate que la pression exercée par le milieu se charge d'éliminer les individus présentant des caractères trop éloignés de la normalité de la population.

On peut définir le concept d'adaptation comme l'ensemble des relations organisme/environnement qui font qu'un être biologique peut vivre et se reproduire dans un habitat donné. Il ne peut être conçu en terme de processus individuel, mais comme une transformation touchant une population entière: l'adaptation est le produit d'un processus évolutif faisant intervenir la sélection naturelle, sans que cette dernière soit forcément adaptative (Lewontin, 1982). Au cours des générations successives, elle résulte d'une modification du patrimoine génétique de la population allant dans le sens d'une optimisation des corrélations entre fonctions physiologiques et morphologiques dans un milieu précis. La possibilité d'adaptation dépend du fait qu'au moins un individu possède des gènes favo- 
rables aux modifications que peut subir l'environnement. Théoriquement, après $n$ générations, les individus "adaptés" devraient seuls se maintenir au détriment de ceux qui n'auraient pas bénéficiés de ce facteur génétique. L'expérience montre qu'en réalité, les gènes «inadaptés 》 ne disparaissent pas totalement mais leur fréquence diminue très fortement. Ils constituent un "polymorphisme génétique résiduel 》 (Ruffié, 1974) qui offre une possibilité élevée d'adaptation en cas de nouvelles modifications des conditions de l'environnement.

Les anthropobiologistes se sont toujours intéressés aux processus adaptatifs (respiratoires, circulatoires, cardio-vasculaires...) en étudiant les réponses de l'organisme aux conditions extrêmes (chaud, froid, altitude). Dans les années 60 , ces recherches ont été coordonnées par le thème "Adaptabilité humaine» du Programme biologique international, et ont permis de mieux cerner, pour des populations de faibles dimensions démographiques, les interfaces entre les structures biologiques des populations et les conditions environnementales. Parmi les études pilotes, on peut citer celles sur Population génétique, niveau de santé et démographie d'une petite population d'Indiens d'Amérique du Sud de Neel (1968), Données alimentaires et démographiques des populations Inuits de Lauglin (1970) ou encore celle de Baker (1969) intitulée Démographie, croissance et dimensions technologiques dans une population de haute altitude.

2. Le concept de population. - Le concept de population n'est pas réservé aux démographes ou aux statisticiens. Il forme l'élément central de la pensée anthropologique moderne qui considère qu'Homo sapiens se situe dans une dynamique évolutive constante et qui étudie à la fois les processus évolutifs et la diversité à l'intérieur de l'espèce. Pour comprendre la manière dont les populations évoluent en se trans- 
formant, il convient d'intégrer des paramètres tels que l'état de sociabilité, la taille, les limites ou encore la perméabilité entre groupes humains (SauvainDugerdil, 1996). Pour l'anthropologie, la description de la réalité biologique d'une population dépend de facteurs culturels et sociaux situés "en amont» de celle-ci. Le passage du concept de «type»- en tant qu'élément de référence à un groupe homogène - à celui de «population» - comme expression de la diversité intragroupe - a provoqué une rupture épistémologique. En effet, l'analyse d'un processus reposant sur une description individuelle et destiné à mettre en valeur les différences entre «types» est remplacé par un autre processus cognitif qui intègre la notion de variabilité obligeant les anthropologues à étudier le niveau collectif. Ce changement d'échelie a fait apparaître de nouveaux problèmes conceptuels et méthodologiques, et a imposé une réflexion approfondie sur le concept de population.

A) Le concept de population biologique. - $\mathrm{Au}$ niveau individuel, chaque homme est inséré dans une structure formée par les relations qu'il entretient avec d'autres hommes : parenté, alliance, relations sociales, économiques, politiques...

La première de ces relations est la parenté. Celle-ci est la plus immédiate puisqu'elle précède déjà notre naissance. L'intensité des relations d'apparentement entre tous les individus d'une population permet de définir une expression biologique de celle-ci. La population peut avoir soit une structure simple, c'est-à-dire que les individus sont reliés entre eux par une structure de parenté ou d'alliance, soit une structure beaucoup plus complexe lorsque leur identification au groupe se fait sur des critères d'appartenance géographique ou professionnelle. Dans les populations ayant les structures démographiques et sociales les plus simples (comme le campement) et pour 
lesquelles nous possédons une bonne connaissance des liens de parenté qui relient les individus entre eux, on peut aisément calculer la consanguinité moyenne et voir l'évolution de celle-ci au cours du temps.

Pour les généticiens des populations, la «population " est définie comme un ensemble d'individus ayant un pool génique commun. Pour voir l'évolution génétique d'une population, on examine l'écart de la fréquence génique observée par rapport à une loi théorique de stabilité du pool génique qui assurerait une transmission régulière et immuable $\mathrm{du}$ patrimoine génétique de génération en génération. C'est le modèle théorique de Hardy-Weinberg qui, par sa rigueur formelle, a permis de construire la " population " comme un objet d'étude. Ce modèle stipule qu'un certain nombre de conditions doivent être remplies pour assurer la stabilité du pool génique au cours du temps. La première, l'unité de temps, sous-entend que tout individu doit appartenir à une génération $(g)$ et trouver un conjoint au sein de cette génération (ce qui implique que ses propres parents et ceux de son conjoint doivent appartenir à la génération précédente $(g-1)$. Ce qui d'ailleurs n'est pas le cas pour les mariages entre oncle et nièce ou entre cousins de degré inégal, puisqu'il y a chevauchement entre générations. La seconde condition suppose qu'il n'y ait pas de migrations et la troisième nécessite l'absence de mutations génétiques. La quatrième implique qu'il n'existe pas d'avantage sélectif pour un gène donné et qu'en conséquence il n'y ait pas de fécondité différentielle. La cinquième des conditions est la panmixie, c'est-à-dire que les unions entre les individus doivent s'effectuer au hasard. La dernière des conditions étant que la population ait un effectif illimité.

B) La dérive génique dans les petites populations. Le problème de la dimension de l'effectif est primordial, car une population comprenant peu d'individus 
va se reproduire d'une manière singulière du point de vue génétique. Les individus très féconds auront un poids génétique considérable alors que les individus peu féconds verront leurs gènes disparaître rapidement au cours des générations. Il s'agit du phénomène de dérive génique qu'on peut observer à partir de reconstructions généalogiques. Les plus anciens individus connus dans ces chaînes généalogiques sont les «fondateurs». C'est à partir d'eux que l'on peut calculer la distance généalogique entre deux individus ou groupes d'individus (c'est-à-dire une distance génétique basée sur une probabilité d'identité génétique) de même que leur probabilité d'origine des gènes. Ainsi, dans le cas d'une population célèbre chez les généticiens des populations (les indiens Jicaques du Honduras), fondée il y a plus d'un siècle par cinq individus et qui est demeurée isolée jusqu'à une époque récente, Chapman (1971) a calculé la probabilité d'origine des gènes pour cinq générations successives. Il a pu en déduire deux constatations : d'une part, le changement de la structure génétique de la population aux générations successives est le produit direct de la fécondité et de la mortalité infantile différentielles et, d'autre part, l'immigration d'individus à la quatrième génération, même faible, accélère très fortement la transformation génétique de la population.

C) Le concept d'isolat. - La spécificité de l'anthropologie est de se démarquer de la génétique des populations en montrant in situ comment les populations humaines se présentent dans la réalité. Ainsi, parmi les problèmes liés à l'application des modèles de la génétique des populations sur des populations réelles, le premier est sûrement celui relatif au principe d'unité de temps et d'espace. On s'aperçoit vite que les problèmes posés par l'unité de temps sont relativement simples à surmonter lorsque 
l'on prend certaines précautions méthodologiques. Il faut, par exemple, ne pas oublier qu'il y a dissociation entre temps chronologique et temps généalogique et donc apparition de problèmes méthodologiques pour comparer entre elles les lignées cognatiques et agnatiques. Par contre, on constate qu'il est plus délicat de délimiter une unité spatiale, car définir une population implique de lui prêter des attributs spécifiques (limites géographiques, composition sociale, critères démographiques...). D'autant que les caractéristiques d'une population ne sont jamais fixes et intangibles car elles varient au cours du temps. Ceci souligne les problèmes rencontrés lorsque l'on tente d'intégrer les deux niveaux d'analyse qui sont à la base des études comparatives : l'intrapopulationnel et l'interpopulationnel. Le problème méthodologique de l'anthropobiologie est donc double puisqu'il se situe autant dans la définition de l'objet de connaissance que dans celui de son niveau d'analyse.

Il convient donc de déterminer quels sont les critères qui permettent d'apprécier les limites d'une population, que celles-ci soient d'ordre géographique ou non. En réalité, ces limites dépendent souvent de facteurs culturels. Par exemple, on utilise fréquemment l'aire matrimoniale comme marqueur de la population génétique. Cette aire matrimoniale correspond à la zone géographique à l'intérieur de laquelle s'effectue au moins $80 \%$ des mariages. Dans la pratique, on utilise fréquemment les distances comme marqueur et on retient les individus se mariant à l'intérieur d'une zone comprenant $80 \%$ de l'ensemble des unions. La population sera qualifiée d' " ouverte " ou de "fermée » suivant que le choix du conjoint s'effectuera préférentiellement à l'intérieur (endogamie) ou à l'extérieur (exogamie) de l'espace qu'elle occupe. Plus l'aire de choix du conjoint sera réduite, plus la population sera considérée comme endogame. 
Le mode de circulation des gènes dans les groupes humains peut donc se classer suivant deux grands modèles (Wright, 1946) :

- le modèle insulaire dans le cas de populations finies et discontinues ;

- le modèle de voisinage dans le cas de populations infinies et continues.

Dans le modèle du voisinage, l'isolement se fait par la distance: plus la distance entre les lieux de naissance entre deux individus est élevée, plus la probabilité qu'ils ont de se marier est faible. Ce modèle a été vérifié dans de nombreuses populations (Boyce, 1967; Yasuda, 1975; Imaizumi, 1986) sauf lorsqu'il existe des règles particulières d'alliance entre groupes comme l'homogamie sociale ou religieuse. Néanmoins, si le modèle d'isolation par la distance de Wright est pertinent pour les populations rurales de la période préindustrielle, il est beaucoup moins performant pour les populations actuelles. Pour ces dernières, la distance n'est plus un facteur d'isolement à cause du développement des communications ; elle est remplacée par d'autres facteurs (sociaux, communautaires, religieux...) qui agissent pour maintenir des barrières génétiques entre groupes.

Le modèle « insulaire » renvoit au concept d'isolat, dont la définition est due au Suédois Wahlund (1928). Il s'agit d'une unité populationnelle définie comme étant un groupe de faible effectif ayant de très rares échanges génétiques avec l'extérieur du groupe durant un nombre important de générations. Pour calculer l'isolat, Wahlund s'appuyait sur le rapport existant entre, d'une part, la dimension de la population, et d'autre part, le nombre de mariages entre parents au $4^{e}$ degré (cousins germains). Par la suite, Sutter et Tabah tenteront de formaliser une « méthodologie de l'isolat » (1957) en intégrant des paramètres proches de la loi de Hardy-Weinberg: effectif et limites de la 
population, migrations, niveau de consanguinité et de fécondité et enfin choix du conjoint.

Lors d'un colloque sur l'étude des isolats, les anthropologues avaient discuté le concept de population à la fois au niveau théorique et à celui de son application sur des populations réelles (Jacquard, 1977). Dans un premier temps, la recherche sur les « isolats » s'est effectivement avérée fructueuse pour vérifier des modèles génétiques sur des populations, puisqu'on a pu mettre en lumière le fonctionnement des barrières géographiques ou culturelles. Puis, avec le développement de nombreuses recherches en anthropologie démographique dans les années 60 et 70 , 1' "isolat» s'est révélé être davantage un concept théorique qu'une réalité, car de nombreux travaux ont montré que le modèle d'isolation génétique, du moins tel qu'il avait été conçu par les généticiens des populations, ne s'appliquait généralement pas à la grande majorité des populations humaines. Les véritables isolats géographiques ou culturels sont très rares et l'ensemble de l'humanité se caractérise plutôt par une pratique d'échange d'individus (donc de gènes) entre groupes.

D) Les concepts d'endogamie et d'exogamie biologiques. - Lorsque l'anthropologue étudie une population, sa première préoccupation est de saisir le niveau d'homogénéité génétique de celle-ci en mesurant son niveau d'endogamie. Il s'agit cependant d'un concept d'emploi difficile car la grande majorité des populations humaines sont, comme nous l'avons vu, des populations génétiquement ouvertes. C'est le mariage qui se présente alors comme le marqueur essentiel pour appréhender l'homogénéité ou la diversité de la population.

Ce concept d'endogamie biologique est déjà ancien puisqu'en 1968, Louis Henry y faisait implicitement référence dans sa définition du cercle de population : 
a / le cercle se forme à une date déterminée et nul ne peut y entrer après cette date;

$b /$ tant que le cercle subsiste, les membres du cercle ne se marient qu'entre eux.

Un des problèmes majeurs sera de définir le niveau géographique pris en considération (campement, hameau, paroisse ou commune, canton, région). Dans le cas du modèle continu de population, c'est-à-dire lorsque les populations étudiées sont insérées dans un continuum humain, il devient nécessaire de déterminer l'effectif efficace théorique, c'est-à-dire le nombre d'individus dans la population qui vont véritablement participer à la reproduction du groupe (CavalliSforza, 1971) et leur aire matrimoniale géographique puisque nous sommes en présence de populations génétiquement «ouvertes ». Souvent, les auteurs considèrent que les populations qui ne sont pas incluses dans l'aire d'étude sont exogames et qu'elles correspondent au "monde extérieur ». Cette démarche est réductionniste puisqu'elle ne prend pas en compte le processus de réenchaînement d'alliance (c'est-à-dire des échanges continuels d'individus entre lignées, indépendamment des critères d'appartenance géographique), pourtant fréquent dans le monde rural européen. Dans ce cas, l'adéquation entre limite territoriale et taille de la population devient primordiale puisque c'est l'appartenance géographique - et non la parenté - qui fait qu'un individu sera ou non considéré comme membre de la communauté. Le facteur géographique est ici un critère explicatif insuffisant puisque les limites administratives (communales ou cantonales) se révèlent arbitraires en ne rendant compte ni de la nature des barrières qui isolent une communauté où, au contraire, qui peuvent la fragmenter. Pour sortir de cette impasse, il convient d'adopter le concept d'isolement par la distance - celle séparant les lieux de naissance des conjoints - 
comme marqueur d'endogamie ou d'exogamie. Toutefois, la mesure de la distance reste problématique car elle se révèle fonction des moyens de locomotion disponibles (donc de la période historique considérée), mais aussi de la nature du terrain. Par exemple, pour les populations montagnardes (Lasker, 1972), la distance à vol d'oiseau ne présente pas une bonne correspondance avec le temps de parcours entre deux points.

Cette question souligne les limites des études dans lesquelles une population est présentée comme un objet « homogénéisable » alors que la réalité montre des populations hétérogènes et fragmentées. Que deviennent alors l'endogamie, la distance matrimoniale moyenne ou même le coefficient de consanguinité d'une population, si celle-ci est constituée de plusieurs 《sous-populations» réunies arbitrairement pour former une unité géographique et/ou administrative ? Comment rassembler sous une même « identité » biologique des agriculteurs vivant en communauté familiale et pratiquant des règles d'endogamie sociale précises associées par exemple au mode de dévolution des biens, avec des commerçants ou des fonctionnaires obéissant aussi à des règles sociales correspondant à d'autres logiques?

Dans le cas des anciennes populations rurales, le modèle d'isolation par la distance s'appuyait sur l'idée que les individus avaient tendance à passer toute leur vie au même endroit (concept de sédentarité), de la naissance à leur mort. Le mariage exogame, seule possibilité de migration géographique, portait souvent sur une faible distance et concernait généralement les femmes.

3. Alliance et parenté. - Le mariage est un préalable au fait familial. Il repose sur un donné biologique de base (un homme et une femme qui s'unissent pour produire des enfants) qui induit à son tour des 
liens de parenté (relation parents-enfants ou frèresœur).

Toutes les sociétés humaines sans exception ont été régies par la parenté. Elle engendre une solidarité multifonctionnelle à laquelle on peut associer mariages, héritages, cultes... On peut s'interroger sur la nature du lien qui unit les Hommes entre eux ? Tout d'abord, nous devons constater qu'il a une double nature: cette parenté peut être consanguine ou affine: la consanguinité étant le domaine de la parenté et l'affinité celui de l'alliance. Ces derniers sont des liens parallèles aux liens naturels de la consanguinité qui parfois s'excluent, parfois se superposent. Chaque société aura d'ailleurs à nommer la multiplicité de ces liens de nature différente puisqu'ils sont fondés sur le sang dans la relation mère/enfant ou sur l'alliance dans la relation homme/femme).

A) L'alliance. - Morgan (1871) avait constaté que dans certaines sociétés les hommes et les femmes s'appellent «frères » et «sours» sans qu'ils en possèdent le lien biologique.' Il avait conclu en l'existence d'une promiscuité et d'un matriarcat primitifs, le seul lien tangible existant étant le lien mère-enfant. Les faits infirment ces suppositions puisque les sociétés les moins développées technologiquement (comme les Aborigènes d'Australie) semblent être celles qui ont les systèmes d'alliance à la fois les plus complexes et les plus sophistiqués pour éviter en même temps une forte endogamie (repliement du groupe) et une exogamie absolue (éclatement ou dissolution du groupe).

a) Le choix du conjoint. S'il sert à consolider la relation sexuelle et à assurer la socialisation des enfants, le mariage est avant tout un acte social qui dépend de l'organisation de la population. Dans les sociétés que l'on nomme «à structures élémentaires de parenté » (parce que chaque individu a une posi- 
tion sociale déterminée dès sa naissance), la place de l'individu dans le groupe induit à la fois son type d'activité, sa résidence et le type d'alliance qu'il peut pratiquer : ce sont les groupes de parenté qui structurent l'organisation sociale (Lévi-Strauss, 1949). De ce fait, les célibataires et les couples sans enfants (surtout les femmes dans les PVD) sont marginalisés et même déconsidérés dans les sociétés où le culte des ancêtres tient une grande place.

Dans nos sociétés contemporaines, le choix du conjoint semble aléatoire, comme guidé par le hasard ou les circonstances de la vie (bal, amis, vacances, bureau...). Si nous ne sommes plus dans le domaine des structures élémentaires de parenté, d'autres paramètres plus nombreux interviennent (travail, amis, loisirs) (Girard, 1964 ; Imaizumi, 1986) ; c'est ce que Lévi-Strauss (1949) nomme les structures complexes.

L'anthropologue Robin Fox (1972) rappelait que les principes de base des systèmes de parenté sont les suivants :

- Principe $1:$ les femmes procréent les enfants;

- Principe 2: Les hommes fécondent les femmes;

- Principe 3: les hommes détiennent le pouvoir;

- Principe $4:$ les proches parents ne s'accouplent pas entre eux.

A partir de ces principes élémentaires (procréation, fécondation, exercice de l'autorité et prévention de l'inceste) qui correspondent tous à d'évidentes et nécessaires interactions entre l'organisation sociale et biologique, on comprend que la diversité peut être grande dans les modes de choix du conjoint. Dans les sociétés fondées sur une économie de subsistance, il n'y a pas de main-d'œuvre salariée et ce sont les groupes familiaux qui assurent la production alimentaire. Tout le monde paraît devoir se marier vite et avoir rapidement des enfants; mais leur nombre dépend de facteurs économiques et culturels. Le mariage est 
alors un système d'attribution des femmes aux hommes, sans que le choix personnel soit de réelle importance. Il a beaucoup plus de sens pour les familles que pour les individus directement concernés, dans la mesure où il intègre des individus dans les lignages et qu'il permet de conclure des alliances entre lignées. Le mariage n'est pas, tout du moins au départ, une affaire privée car les règles qui contrôlent les alliances matrimoniales sont dictées par des impératifs familiaux.

Dans les sociétés à structuration économique et sociale plus complexe, si quelques comportements demeurent récurrents comme les choix matrimoniaux des hommes économiquement aisés qui vont vers des femmes plus jeunes qu'eux et qui possèdent des potentialités reproductives élevées (Jaffe, 1995), les règles de mariage sont davantage soumises à des choix individuels. La règle la plus connue du contrôle social de la sexualité est celle de la répression de l'inceste. Dans l'espèce humaine, les enfants ne se marient ni entre eux, ni avec leurs parents. Lorsque cette loi est violée, il y a inceste. Pour Lévi-Strauss (1949), c'est cette interdiction de l'inceste qui est la marque de la culture en portant en elle la rupture avec le naturel où tout est instinctif. Par sa complexité et son universalisme, le problème de l'inceste - dans la mesure où il n'est pas aussi simple que le pensent les ethnologues et sexologues (pour lesquels il existerait peu d'attrait entre parents proches) ou les psychanalystes (pour lesquels il en existerait au contraire beaucoup) - intéresse bien sûr les anthropologues. En effet, la prohibition de l'inceste serait inutile si elle n'existait pas. D'ailleurs, la littérature (CEdipe), l'histoire (Néron et sa mère Agrippine) et la criminologie attestent de sa réalité.

b) Exogamie/endogamie. Le moyen le plus radical mis en œuvre par les groupes humains contre l'inceste est la pratique de l'exogamie, coutume selon laquelle 
on doit se marier hors du clan (ou du groupe). Dans les sociétés à structuration simple, la descendance est souvent unilinéaire, c'est-à-dire qu'elle se transmet soit par les hommes, soit par les femmes. Le groupe de descendants unis par ce principe s'appelle un lignage. Les lignages, souvent organisés en clans, sont généralement exogames (interdiction faite aux membres d'un même lignage de se marier entre eux). L'individu est alors obligé de chercher un conjoint hors du village, ce qui crée des liens forts entre clans et entre villages. L'exogamie permet donc d'entretenir la paix avec les voisins et d'accroître les poids politiques des individus ayant arrangé de bons mariages pour leurs descendants, surtout si ceux-ci sont nombreux. La pratique de l'exogamie permet à la fois le développement de nouvelles alliances et le renouvellement systématique des anciennes.

Il existe bien sûr des groupes qui rejettent l'exogamie systématique; le cas le plus connu est celui du mariage «arabe ». En effet, les tribus arabes ont toujours attaché la plus grande importance au principe de descendance patrilinéaire. Il s'agit d'une société segmentaire où chaque tribu se considère comme un vaste lignage divisé en segments fondés par les fils (puis par les petits-fils, etc.) du «fondateur ». Chaque section portera elle-même le nom de son fondateur putatif. La direction du groupe appartient à la lignée paternelle, et lorsque celui-ci campe, les tentes sont plantées selon un ordre indiquant les degré de parenté agnatiques entre les chefs de famille: les fils près du père, les cousins germains plus loin, etc. Lorsqu'il faut décider un mariage, ce sont les hommes du lignage qui décident. La volonté est que les mariages se fassent dans une parenté très proche, à l'exclusion des frères et des sœurs. Ainsi, la cousine germaine patrilinéaire est considérée comme l'épouse préférentielle. Tout individu désirant épouser une jeune fille doit regarder le nombre de prétendants 
possibles qui pourraient se mettre sur les rangs parce qu'ayant un degré de parenté plus rapproché. Ce système renforce la cohésion lignagère. Selon l'anthropologue L. Mair (1971), ce type de mariage a autant d'avantages et de sécurité que le système exogamique. Chez les Bédouins il est plus important d'assurer une très forte cohésion du groupe que de développer des alliances avec d'autres tribus.

L'endogamie est une règle rigoureuse qui impose qu'on se marie dans un groupe déterminé, interdisant de chercher un conjoint hors du groupe. Il ne s'agit pas de conserver la cohérence du groupe, mais de dresser une barrière face à l'extérieur. L'endogamie est associée à une stratification sociale. Citons le cas des castes de l'Inde où les intouchables exercent des métiers associés à la souillure: bouchers, tanneurs, blanchisseurs, balayeurs... Les barrières sociales sont très rigides puisque l'appartenance à la caste est héréditaire et qu'il est interdit aux individus de se marier en dehors de celle-ci.

A l'inverse de l'endogamie qui contribue à une reproduction des groupes sociaux très compartimentée, l'exogamie indique des transformations au sein de la société. Elle s'évalue généralement en mesurant la distance socioprofessionnelle et socioculturelle séparant les conjoints en se basant sur les professions (ou l'appartenance religieuse) des pères des époux ou entre le mari et son beau-père (Segalen, 1971). Le concept d'endogamie sociale (ou homogamie) s'avère surtout opératoire dans une population où les strates sociales évoluent très lentement au cours du temps, car dans le cas d'une société en transformation rapide - comme cela est le cas dans les sociétés industrialisées à partir du milieu $d u \mathrm{XIX}^{\mathrm{e}}$ siècle - son application devient mal aisée. 'D'ailleurs, l'emploi du concept d'homogamie a même été fortement critiqué dans son application par la sociologie (Singly, 1987). En effet, considérer le statut socioprofessionnel de l'individu 
au moment de son mariage ou celui des parents des deux conjoints ne permet pas d'intégrer les potentialités de promotion sociale des maris et ne fait pas intervenir les métiers en émergence dans la société. Pourtant, cette « mesure » demeure utile pour évaluer l'endogamie professionnelle lorsque les frontières sociales sont rigides et transcendent l'endogamie géographique (médecins, notaires...).

Enfin, on remarque que la règle d'endogamie est beaucoup plus difficile à faire respecter que la règle d'exogamie : la première permet de conserver la cohésion du groupe mais n'apporte généralement pas d'avantages à l'individu, à l'inverse de la seconde qui autorise la promotion sociale.

B) La parenté. - La parenté est un domaine qui intéresse non seulement l'anthropologie biologique, mais aussi la sociologie (institution de la famille et du mariage), la génétique (identité génétique des individus) ou l'ethnologie (système de relations sociales).

Selon la définition la plus communément admise, la «parenté consanguine» est la relation entre parents. Le champ de celle-ci est extrêmement vaste puisqu'elle comprend pour chacun d'entre nous des milliers d'individus. Dans notre société, nous connaissons généralement notre parenté immédiate qui dépasse rarement les descendants de nos deux couples de grands-parents (oncles, tantes et cousins). Cette limitation du champ de la parenté rend bien compte de l'idée qu'elle repose sur un principe de reproduction génétique. Elle illustre bien la conception que notre société se fait de l'identité et qui découle de notre connaissance $\mathrm{du}$ principe de la reproduction biologique (nos deux parents participent de manière équivalente à la constitution de notre identité biologique) ; mais cette conception de la construction identitaire est loin d'être universellement admise. En effet d'autres sociétés expriment des représentations des 
principes de reproduction et d'identité différents, par exemple, lorsqu'ils croient que l'homme et la femme contribuent de manière très inégale à la formation de l'enfant : l'un donne le corps, l'autre l'âme, ou l'un le sang et l'autre les os. Chez certains, le père apporte une graine et la mère fait fonction d'incubateur ; chez d'autres, au contraire, l'enfant est entièrement conçu par la mère, le père fait seulement fonction de déclencheur du processus.

Ceci montre combien le concept de consanguinité et l'ordre généalogique sur lequel il repose peuvent être spécifiques à chaque société. Un parent consanguin est un individu défini comme tel par la société dans lequel il vit. Ce n'est pas toujours une consanguinité génétique, même si dans la plupart des sociétés, parenté biologique et parenté sociale ont tendance à se superposer.

La définition des systèmes élémentaires de parenté est finalement assez simple. Elle consiste à trouver la catégorie de femmes qu'un individu doit épouser et celles qui lui sont interdites. On constate que plus les sociétés sont petites, plus le jeu des règles matrimoniales est complexe. La terminologie de la parenté distribue tous les membres du groupe dans diverses catégories avec lesquelles le mariage est possible ou non. Dans le cas où il est prescrit d'épouser quelqu'un de sa parenté, il s'agit d'un mariage préférentiel. Quand les donneurs de femmes sont aussi les preneurs, il s'agit d'un échange direct, symétrique, que l'on nomme "échange restreint".

La forme la plus simple est l'échange systématique des sœurs entre deux frères qui deviennent ainsi deux fois beaux-frères; elle se complexifie selon la symétrie ou l'asymétrie de l'échange (patrilinéaire, matrilinéaire, croisé, parallèle). Dans le système le plus simple d'échange restreint, l'homme doit prendre femme là où son père a pris femme, comme dans le système dit Kariera où l'individu doit épouser sa cou- 
sine croisée bilatérale. Il peut aussi se marier avec sa cousine croisée au deuxième degré, c'est-à-dire là où son grand-père a pris femme. Par contre, quand les preneurs de femmes ne sont pas les donneurs, l'échange est asymétrique et nécessite la présence d'au moins trois groupes. C'est une forme beaucoup plus complexe : ( $\mathrm{A}$ donne à $\mathrm{B}$, qui donne à $\mathrm{C} .$. qui donne à $\mathrm{A})$.

Si ces systèmes à structure de parenté élémentaire s'observent dans les populations dites « traditionnelles », il existe aussi des systèmes complexes reposant sur des règles négatives de mariages, puisqu'elles définissent les parents que l'on ne doit pas épouser, ce qui est le cas des sociétés occidentales.

\section{II. - Les théories et méthodes démographiques}

La démographie a pour objectif d'étudier les populations humaines en rapport avec leur renouvellement par le jeu des naissances, des décès et des mouvements migratoires (Pressat, 1982). Elle a pour vocation de décrire l'état des populations (les effectifs, par catégories selon le sexe, l'âge, l'état matrimonial, le ménage, etc.), et les phénomènes qui influent sur cet état et son évolution (natalité, nuptialité, mortalité, migration). Mais la démographie est aussi revendiquée comme une science sociale qui, au-delà des approches quantifiées, cherche non seulement à retracer des processus mais aussi à les comprendre, à les expliquer au regard des modes d'organisations des sociétés et des comportements humains. Comme toute science, la démographie s'appuie sur des méthodes et des techniques d'analyse qui lui sont propres et que nous allons exposer succinctement.

1. Connaître les théories. - Les grandes théories aujourd'hui encore opératoires en démographie sont 
en nombres limitées et peuvent se résumer à la question du rapport entre la population et les ressources, et au problème de la transition démographique.

A) La relation population/ressources. - Cette relation entre l'effectif de la population et les ressources disponibles dans son milieu de vie a fait l'objet d'importantes controverses et elle reste encore aujourd'hui d'actualité. Elle s'applique aussi aux recherches qu'effectuent les anthropologues, particulièrement celles qui abordent les questions du peuplement des territoires par les groupes humains et les problèmes d'adaptations aux différents milieux qui en découlent.

Le premier à avoir laissé une empreinte aussi forte sur la démographie est Thomas R. Malthus, qui en 1798, a publié son ouvrage Essai sur le principe de la population, dont les conclusions sont encore aujourd'hui l'objet d'interprétations. Sa théorie affirme que les êtres humains ont la propension à se reproduire jusqu'à devenir tellement nombreux qu'ils finissent par disposer de ressources insuffisantes. Il affine son propos en remarquant qu'il existe une différence de nature entre l'accroissement des hommes et celui des ressources. En effet, selon ses observations, la "race humaine » suit une progression géométrique $(1,2,4,8,16 \ldots)$ alors que les "moyens de subsistances » obéissent à une progression arithmétique $(1,2,3,4,5 \ldots)$.

Ces questions ont suscité l'intérêt des mathématiciens qui, en essayant de quantifier ce processus, ont fait progresser la réflexion en modélisant les théories énoncées. La théorie de Malthus s'est trouvée mise en examen et les travaux de biologistes et de mathématiciens ont montré que la population ne pouvait croître indéfiniment et qu'elle finissait par atteindre une limite. Parmi les chercheurs, on retiendra le nom de Raymond Pearl qui, s'appuyant sur des données de 
populations réelles, démontre l'existence d'une limite à la croissance de la population, mettant ainsi à jour une courbe en $S$, appelée courbe logistique. Cette transcription graphique d'une loi mathématique montre bien comment une population peut évoluer, en croissant d'abord très rapidement, puis plus lentement jusqu'à atteindre une limite supérieure.

C'est à partir de cette démonstration que s'instaure la discussion sur la théorie malthusienne. Quelles relations y a-t-il entre la croissance de la population, le progrès technologique et l'augmentation des productions agricoles et/ou industrielles qui en dépend, et comment déterminer quelle sera la population limite dans une situation donnée pour une population préalablement définie. C'est de là que vient le terme de carrying capacity qui correspond à la population maximale pouvant vivre sur un territoire déterminé, avec des ressources en quantité limitée. Cela revient en fait à examiner la croissance d'une population connue au cours d'une période temporelle bien définie. Si l'on veut raisonner sur une période de temps long, on assiste alors à une succession de cycles, c'est-à-dire de situations qui se reproduisent à l'identique, avec peut-être seulement des différences dans l'intensité des phénomènes.

Cette situation de limite a été souvent discutée par les démographes, elle correspond à ce piège malthusien aussi appelé trappe malthusienne qui veut que les ressources augmentent sous la dépendance de progrès techniques, induisant ou permettant alors (selon la finalité que l'on souhaite donner à ce phénomène) une augmentation de la population qui à son tour dépassera les ressources disponibles pour la survie de cette population. Le démographe Kingsley Davis (1991) pense que la question des subsistances n'est pas le seul paramètre qui intervient dans la limitation. de la croissance de la population, mais qu'il faut aussi tenir compte des comportements des populations 
humaines. De son côté, Noël Bonneuil (1997) assure que le niveau technologique n'est pas directement une conséquence de la pression démographique, mais qu'il faut plutôt envisager, dans la relation population/ressources, le rôle que peuvent jouer les individus dans le choix de stratégies adaptatives.

Pour les pays en développement, Ester Boserup (1965) soutient l'idée contraire à Malthus, à savoir que c'est la croissance démographique qui génère les progrès économiques et sociaux et par conséquent une augmentation des ressources. Cette thèse permettrait d'expliquer pour le monde rural et agricole des pays en développement, en particulier en Afrique, que la faible densité de population ne pousse pas à des changements dans les modes d'utilisation du sol. En fait, les théories de Malthus ou de Boserup se situent souvent au croisement entre paramètres économiques et démographiques et la tendance actuelle des démographes est de constater les complémentarités entre ces deux théories beaucoup plus que des oppositions.

B) La transition démographique. - Théorie plus récente, elle est très fréquemment employée pour analyser l'évolution des populations dans le monde contemporain. La transition démographique peut se définir comme la succession logique de phases historiques que traverse toute population au cours du processus d'accès à la modernité. Le concept de transition démographique désigne donc le passage «à l'occasion d'un processus de modernisation globale, et au bout d'un certain délai, d'un régime traditionnel d'équilibre démographique à mortalité et fécondité fortes, à un régime moderne d'équilibre à mortalité et fécondité basses 》(Chesnais, 1986).

Sur la figure 1, on s'aperçoit que les périodes temporelles 2 et 3 correspondent à la durée du processus de transition qui se caractérise par une diminution de la mortalité et de la fécondité et parallèlement par 


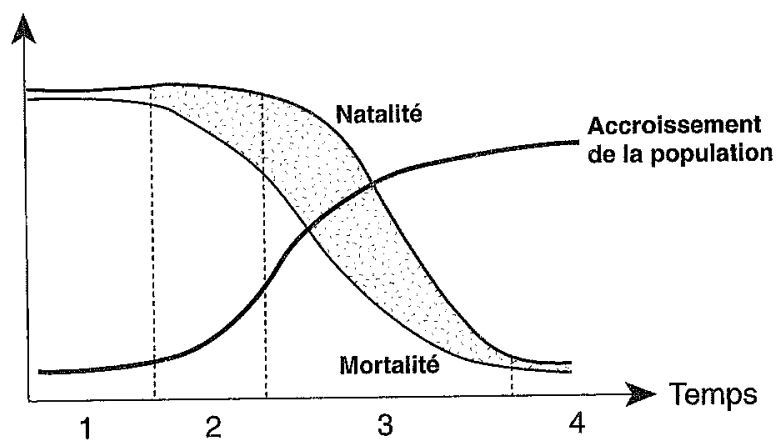

Fig. 1. - Les différentes étapes de la transition démographique.

une augmentation de la croissance naturelle. C'est seulement au cours de la période 3 que l'on assiste au début de la diminution de la fécondité.

Dans les pays européens, en matière de baisse de la mortalité, certaines dates marquent souvent des ruptures dans l'évolution de la mortalité générale. La plupart des auteurs s'accordent pour admettre que la fin $\mathrm{du} \mathrm{XVII}^{\mathrm{e}}$ et la première partie du XIX $\mathrm{X}^{\mathrm{e}}$ siècle ont vu s'effectuer une baisse lente de la mortalité, due pour grande partie aux progrès agricoles. La décennie 1870/ 1880 est la période charnière avec les découvertes de Pasteur. Cette révolution médicale de la fin du $\mathrm{XIX}^{\mathrm{e}}$ siècle est suivie par deux dates ou périodes clefs. Ce sont respectivement la période qui commence après la guerre de 1914, avec la diffusion de l'éducation sanitaire, puis celle d'après 1945 avec la découverte et la généralisation des antibiotiques.

Le recul de la mortalité dépend donc à la fois de la médecine, de l'hygiène, du niveau de vie et les causes de la baisse s'articulent principalement autour du couple techniques sanitaires/développement socioéconomique. Les conséquences sont l'augmentation du 
rythme de croissance de la population et son rajeunissement, favorisant ainsi une nouvelle régulation et provoquant des situations d'exode rural liées aux transformations sociologiques des populations. En revanche, la baisse de la fécondité est un phénomène beaucoup plus complexe, et son interprétation dépend tout autant de facteurs socioéconomiques que culturels.

Toutefois, trois éléments majeurs peuvent émerger de la diversité des situations :

- le caractère préalable de la baisse de la mortalité dans toutes les situations, à l'exception de la France ;

- l'existence de deux phases dans le contrôle de la fécondité (restriction des mariages, puis des naissances);

- et enfin, d'une façon plus générale, l'influence du contexte de modernisation sur la baisse séculaire de la fécondité.

Dans le cas d'une forte croissance de population, signalons l'importance $d u$ phénomène migratoire envisagé comme mécanisme de régulation démographique et réducteur de tensions économiques et sociales. Très schématiquement on peut suggérer une suite de causalité : baisse de la mortalité, augmentation de l'effectif de population, adaptation aux ressources, contrôle de la fécondité, migration.

Il n'existe pas de modèle unique de transition démographique et il est possible de distinguer trois grands types de transition (les pays industrialisés européens, les grands pays d'immigration et les pays en voie de développement).

- Pour les pays européens, la croissance naturelle plafonne à $2 \%$ par an et la durée de la transition est généralement longue (de 75 ans jusqu'à deux siècles).

Il existe quelques différences liées à la géographie et à l'histoire du développement, les progrès sanitaires 
et la diminution des naissances s'étant organisés en un gradient du Nord-Ouest vers le Sud et l'Est du continent.

Le cas français est totalement atypique avec une baisse de la natalité très précoce et un profil plat de croissance naturelle proche de zéro. On pourrait attribuer ce contrôle de la fécondité à la révolution des idées qui s'est fait jour dès la fin du XVIII ${ }^{e}$ siècle, avec le recul dans les campagnes du sentiment religieux, au contexte politique qui découle de la révolution française avec l'affirmation de la propriété individuelle, ou bien encore à la question agraire française spécifique (surpeuplement des campagnes, grande extension de la petite propriété familiale).

- A l'inverse, les pays en voie de développement présentent souvent des transitions de courte durée et une forte croissance naturelle allant de $2 \%$ jusqu'à $4 \%$. Certains pays n'ont d'ailleurs pas encore achevé leur transition, car la baisse de la natalité tarde à s'effectuer. Cela provient de la difficulté pour certaines populations à maîtriser leur fécondité. C'est une question qui dépend de nombreux facteurs, en particulier de nature socioéconomique mais aussi culturelle (âge au mariage, polygamie, fait religieux, scolarisation des filles, etc.).

D'une façon générale, quatre catégories de facteurs semblent prépondérant dans l'analyse de la transition dans les pays en développement : sanitaires, économiques, éducatifs et urbains. Certains auteurs vont même jusqu'à remettre en question l'organisation des phases de la transition. Dans certains pays, c'est le développement économique général qui serait à l'origine du déclenchement de la transition et en particulier de la baisse de la mortalité plutôt que la médecine importée (Fargues, 1986).

2. Utiliser les méthodes. - Dans les études de démographie, on procède d'abord au recueil des don- 
nées (sources, observations) et à leur analyse (événements démographiques, analyses transversales ou longitudinales) en effectuant généralement une recherche causale qui impose parfois une collaboration avec d'autres disciplines, surtout lorsqu'il s'agit d'essayer de replacer dans le champ du social les analyses spécifiques conduites sur la structure et l'évolution de la population.

A) Les sources. - Tous les pays effectuent régulièrement des recensements pour dénombrer leur population, en apprécier l'évolution, et en décrire, par unité de résidence, la structure selon les différentes circonscriptions administratives. En France, les recensements s'effectuent en théorie par périodes quinquennales depuis 1836. En outre, tous les événements (naissances, mariages, divorces, décès) sont enregistrés dans des registres d'état civil qui permettent de retrouver pour tout individu une trace de son existence, à travers quelques dates majeures qui jalonnent sa vie. Dans certains pays, il existe aussi des registres qui consignent les migrations.

C'est surtout dans les pays en développement que les statistiques sont incomplètes, car l'instauration de l'état civil est souvent récent et les enregistrements généralement très imparfaits. Les enquêtes complémentaires sont alors très utiles pour ces pays afin d'obtenir soit des informations sur l'évolution démographique de groupes réduits (petites populations rurales, villes) par des passages répétés ou au contraire pour apprécier l'évolution de phénomènes spécifiques à une dimension internationale comme par exemple l'enquête mondiale de fécondité, ou bien plus récemment les enquêtes démographie/santé.

Le recensement permet le dénombrement de la population et l'appréciation de l'évolution de son effectif, ainsi que le classement de la population selon l'âge et le sexe. Il donne également la possibilité 
d'effectuer des études en fonction d'une unité de base qui est le ménage. Quant à l'état civil, il offre, entre autre possibilité, celle de mesurer l'évolution du mouvement naturel en comptabilisant les naissances et les décès.

B) Les événements démographiques. - Pour les démographes, la nuptialité est souvent considérée comme une variable intermédiaire de la fécondité. Dans l'étude de la fécondité, certaines notions sont importantes, comme celle de fécondité naturelle élaborée par Louis Henry (1961) qui correspond à la fécondité d'une population en l'absence de limitation des naissances. Nombreux furent les travaux qui traitèrent des déterminants de la fécondité, en particulier ceux de Bongaarts et Potter (1983) qui, dans l'ensemble des variables intermédiaires, effectuent une hiérarchisation et mettent en évidence le poids plus important accordé à quatre d'entre elles: l'infécondabilité postpartum (période après la naissance d'un enfant où la femme n'est pas fécondable), la contraception, l'avortement provoqué et le célibat.

La mortalité générale s'envisage surtout en relation avec la natalité pour mesurer le niveau d'accroissement d'une population. La mortalité infantile est dépendante des conditions générales de l'environnement dans lequel vit l'enfant et elle est très liée au niveau de développement socioéconomique (exemple des pays en développement). Les déterminants de la mortalité varient selon les époques, les pays, les cultures, les conditions d'environnement naturel et les démographes examinent souvent la mortalité différentielle selon le sexe ou l'activité professionnelle.

La migration est un phénomène spatio-temporel, elle concerne des hommes en mouvement que sont les migrants et son étude fait l'objet de modèles démographiques, surtout depuis les développements récents de l'analyse par des moyens informatisés. Les démo- 
graphes s'attachent à étudier les flux de populations entre deux zones. Il existe une classification des types de migrations (internes, internationales, de travail, matrimoniales, de retraite, temporaires ou viagères) et quelques situations particulières qui concernent les déplacements humains et leurs déterminants sociaux attirent l'attention (les migrations Nord-Sud et les processus d'urbanisation par exemple).

C) Les analyses. - Elles servent à décrire la structure et la dynamique de populations humaines dans l'espace et dans le temps. Le plus souvent, la mesure de phénomènes démographiques implique une nécessaire quantification pouvant aller jusqu'à la construction de modèles hautement sophistiqués. D'autres problèmes, souvent soulevés, sont relatifs à l'échelle d'étude (échantillon, petite population, population de fort effectif, ) et à l'approche choisie (transversale ou longitudinale).

L'analyse démographique se subdivise en analyse longitudinale (par cohorte ou génération) et analyse transversale (par période ou du moment). Dans une cohorte, les deux éléments essentiels qui la caractérise sont l'intensité (nombre événements frappant une cohorte ramené à l'effectif initial de la cohorte) et le calendrier (répartition à travers le temps événements frappant une même cohorte). Un exemple d'analyse longitudinale est l'étude d'une cohorte de naissance sur laquelle on souhaite étudier la fécondité, en supposant l'absence de mortalité et de migration. On prend alors pour un territoire donné, la totalité des femmes nées une même année et l'on observe en continu (généralement par an) le nombre de naissances survenues pour l'ensemble des femmes. Cela permet de faire différents calculs comme ceux des taux ou des indices de fécondité.

A l'inverse, dans l'analyse transversale, on considère les événements pour une année de calendrier ou 
une période de temps déterminée. Dans ce cas, la question de la structure par âge de la population se pose et différents procédés permettent de remédier à cet inconvénient.

Il est bien entendu intéressant d'associer les deux approches, longitudinale et transversale et l'évolution de la fécondité en est un bon exemple où selon l'approche choisie on pourra comparer la descendance finale avec la somme des naissances réduites qui permettent dans le premier cas de dégager des régularités du phénomène et dans un second cas de visualiser les variations plus conjoncturelles. Ces différents points méthodologiques sont évidemment importants chez les démographes, car ils conditionneront ensuite les bases sur lesquelles se feront les prévisions en matière d'évolution de la population, qui est un paramètre très présent dans les études démographiques. Ces différents aspects sont d'ailleurs décrit dans les manuels de démographie (voir par exemple celui de Tapinos, 1985).

\section{III. - Les estimateurs \\ de la structure génétique \\ et de l'évolution biologique}

L'ensemble de l'humanité apparaît composée de populations en interaction les unes avec les autres puisque de nombreux croisements autorisent un brassage génétique et c'est l'intensité du flux génique entre les populations qui assure la plus ou moins grande homogénéité biologique de l'espèce.

Cette dimension génétique est appréhendée grâce aux notions de distance et d'identité. Ceux-ci reposent sur une observation triviale de la réalité : plus les individus sont apparentés, plus ils auront de chance de se ressembler.

Il existe plusieurs systèmes pour calculer la distance séparant Alter d'Ego. 
Dans les anciens systèmes chinois et hébreux comme dans le système romain, le calcul se faisait par gradus, le long de doubles chaînes de consanguinité (ascendante puis descendante). Dans ce système de calcul, on compte les intervalles à partir d'Ego jusqu'à l'ancêtre commun, puis on redescend jusqu'à Alter. Le système canon a remplacé le système romain vers le $\mathrm{VII}^{\mathrm{e}}$ siècle. Il est inspiré par le système germanique dans lequel on comptait la distance entre Ego et Alter selon le nombre de générations les séparant. On compte donc une seule fois la distance reliant Ego à Alter via l'ancêtre commun. Par exemple, les cousins germains sont parents du second degré, car deux générations les séparent: les parents et les grands-parents. Lorsque les chaînes sont de degré inégal, on retient la chaîne la plus longue. Les règles d'interdiction religieuse de mariage entre parents ont varié du cinquième au septième degré dans le monde chrétien (Goody, 1985).

\section{Le coefficient de consanguinité d'un individu.}

Le concept de consanguinité repose sur une recherche d'identité génétique entre deux individus qui produiront une descendance. On dit que deux personnes sont dites apparentées si, parmi les ascendants de l'une, figurent les ascendants de l'autre, ou l'autre luimême (parent-enfant). La transmission simultanée des gènes des deux parents est le départ du processus de formation biologique d'un individu. Lorsque deux individus ne possèdent pas d'ancêtre commun, le coefficient d'identité est nul et leur distance génétique est maximale (Jacquard, 1970).

Sachant que chaque individu reçoit la moitié des gènes de son père et la moitié des gènes de sa mère, ou encore, le quart des gènes de chacun de ses quatre grands-parents, etc., un modèle «mathématique » peut être proposé : si C est l'ancêtre commun de $\mathrm{A}$ et de $B$, alors, la probabilité pour que $A$ reçoive un gène 
de $\mathrm{C}$ séparé par $n$ générations est: $(1 / 2) n$ (Plus l'individu A sera éloigné de $C$, plus la probabilité qu'il reçoive un gène de celui-ci sera faible). De même, la probabilité pour que $B$ reçoive un gène de $C$ séparé de lui par $p$ générations est : $(1 / 2) p$.

La probabilité pour qu'un gène de $\mathrm{A}$ soit identique à un gène de $B$ est ce que l'on nomme le coefficient de parenté $k \mathrm{AB}$ qui peut se calculer par la formule :

$$
k \mathrm{AB}=(1 / 2) n+p+1\left(1+\mathrm{F}_{c}\right)
$$

On peut appliquer ce raisonnement à un cas simple, celui de cousins germains (un couple de grands-parents commun) : si nous prenons l'un des deux gènes porté par A à un locus choisi au hasard, il y a une chance sur deux qu'il lui ait été transmis par son père. $B$ présente la même probabilité. Il en est de même pour le père par rapport à son père (supposé commun à $\mathrm{A}$ et $\mathrm{B}$ ). Il $\mathrm{y}$ a donc $(1 / 2)(1 / 2)=1 / 4$ de chance que chacun des pères ait hérité le gène de leur propre père et donc 1/16 de chance que $A$ et $B$ l'aient reçu de leur grand-père (application de la formule : pour les cousins germains : $k=(1 / 2) 2+2+1+(1 / 2) 2+2+1+=1 / 16=.0625)$. Le facteur $F_{c}$ correspond au coefficient de consanguinité de l'ancêtre $\mathrm{C}$.

\section{Le coefficient de consanguinité d'une population.} - Les premières études concernant le niveau de consanguinité d'une population ont été établies à partir des registres catholiques de dispenses de mariage. Dans ces approches, la fréquence de mariages entre apparentés connus (cousins germains) donne une bonne estimation du niveau de la consanguinité d'une population. Mais il existe d'autre moyens de calculer le niveau de consanguinité d'une population, comme la méthode généalogique et la méthode isonymique.

A) La consanguinité généalogique. - Le calcul de la consanguinité généalogique nécessite de connaître 
au moins les trois générations ascendantes du sujet, c'est-à-dire la parenté comprise au niveau des «cousins issus de germains ». Cette mesure nous donne l'expression de la consanguinité proche.

L'application pratique est la suivante: on calcule la fréquence des différents types de mariages consanguins rencontrés et on les affectent de leur coefficient de parenté $(1 / 8,1 / 16$, etc.) afin de calculer la consanguinité générale par la formule suivante :

$$
\mathrm{C}=1 / 8 f 3+1 / 16 f 4+1 / 32 f 5+1 / 64 f 6+1 / 8 f_{d}
$$

dans laquelle $f$ est la fréquence des mariages entre

$f 3=$ oncle/nièce ou tante/neveu (coefficient de parenté $1 / 8$ ) ;

$f 4=$ cousins germains (coefficient de parenté $1 /$ 16) ;

$f 5=$ cousins germains de degrés inégaux (1/32);

$f 6=$ cousins issus de germains $(1 / 64)$;

$f_{d}=$ doubles cousins germains $(1 / 8)$.

Il existe une autre consanguinité, dite consanguinité éloignée, c'est-à-dire celle concernant les ancêtres beaucoup plus éloignés, mais son calcul nécessite évidemment le recours à des généalogies profondes et souvent difficiles à reconstituer.

B) La consanguinité isonymique. - Une autre méthode a été imaginée par Crow et Mange (1965) pour pallier l'absence de généalogies profondes. Elle repose sur le principe de la transmission des noms de familles (à condition que celle-ci soit patrilinéaire). En général, en Europe, les enfants reçoivent leur nom de leur père (à l'exception de l'Espagne où les individus reçoivent le nom de chacun des deux parents mais ne transmettent que le nom paternel à leur enfants). Au fil des générations, le patronyme devient un «marqueur» du chromosome $Y$.

Lorsque l'on regarde la distribution d'un patro- 
nyme dans un groupe familial donné, on remarque que la probabilité pour que deux personnes prises au hasard portent le même nom - quelle que soit par ailleurs leur relation de parenté effective - est quatre fois plus élevée que la relation de parenté réelle qu'elles présentent dans leur patrimoine génétique pour un locus donné, donc que leur coefficient de parenté.

Partant de ce principe, on peut utiliser les fréquences de mariages entre personnes de même patronyme pour mesurer la consanguinité moyenne d'une population (à condition que chaque patronyme ait la même origine, soit un seul ancêtre par nom). La consanguinité isonyme $F$ est la somme de deux composantes : $F_{r}$ qui représente la part structurelle et $F_{n}$ qui représente la part conjoncturelle.

On part du principe que si les mariages étaient contractés au hasard et qu'il y ait respectivement $p_{i}$ hommes et $q_{i}$ femmes portant le $n$-ième nom, la fréquence avec laquelle ils formeront un couple isonymique est $p_{i} q_{i}$. La contribution de chaque nom au coefficient moyen de consanguinité de la population est alors:

$$
S_{\text {piqi }} / 4
$$

et pour l'ensemble des noms :

$$
\mathrm{F}_{r}=\mathrm{S}_{p q} / 4 \text {. }
$$

Il s'agit bien d'une contribution aléatoire car structurelle et reflétant la composition de la population (plus de garçons et de filles auront le nom $i$, plus grande sera leur probabilité de se marier ensemble).

La composante conjoncturelle repose d'abord sur la fréquence du nombre de mariage isonymiques, c'est-à-dire de couples dont les deux conjoints portent le même nom. Si P est la proportion des mariages isonymiques dans la population, la composante venant des mariages consanguins non aléatoires est : 


$$
\mathrm{F}_{n}=\left(\mathrm{P}-\mathrm{S}_{p q}\right) / 4\left(1-\mathrm{S}_{p q}\right)
$$

$\mathrm{F}_{n}$ représente la contribution conjoncturelle, c'est-àdire qu'elle est fortement influencée par la fréquence des mariages isonymiques: elle exprime donc une volonté de se marier avec quelqu'un qui porte le même nom que soi.

Enfin, la consanguinité isonymique $\mathrm{F}$ a une étroite correspondance avec la somme de ces deux indices:

$$
\mathbf{F}=\mathbf{F}_{n}+\left(1-\mathbf{F}_{n}\right) \mathbf{F}_{r} .
$$

Ce coefficient d'isonymie exprime une consanguinité globale. En regroupant une consanguinité proche et éloignée, elle forme un tout indissociable qui exprimerait un niveau moyen de consanguinité. Néanmoins, cette méthode présente des limites car, contrairement à la méthode généalogique, elle ne peut rendre compte de la présence de sous-populations au sein d'un même ensemble populationnel.

Le choix des différentes méthodes utilisées pour calculer le niveau de consanguinité d'une population dépend de la nature de l'information recueillie. Dans le cas de la consanguinité calculée par la méthode généalogique, la qualité informative va dépendre de la profondeur généalogique. La méthode isonymique contribuera à reconstruire l'histoire génétique de la population, mais, dans la mesure où cette consanguinité ne dissocie pas la consanguinité proche de la consanguinité lointaine, elle servira surtout à montrer les comportements matrimoniaux en terme d'adoption ou de rejet du mariage consanguin au cours du temps.

3. La probabilité d'origine des gènes. - Le concept de "probabilité d'origine des gènes" a été élaboré par la génétique des populations. Il s'avère particulièrement efficace pour mesurer le rythme d'ouverture génétique dans des populations relativement isolées. 
La méthode de calcul de la «probabilité d'origine des gènes " concerne le patrimoine génétique de la population et surtout son changement entre les générations successives. Cette méthode repose sur la reconstruction d'arbres généalogiques, comme dans le cas du calcul de la consanguinité dite "généalogique ». En remontant les chaînes généalogiques, on se trouve toujours arrêté par le manque d'information concernant un certain nombre d'ancêtres pour lesquels on ignore tout de leur parents. En génétique des populations, ces individus sont considérés comme étant des "fondateurs ». Plus le fondateur sera éloigné de la génération considérée, plus son poids génétique sera faible. La probabilité pour qu'un gène quelconque $\mathrm{W}$ chez un individu $i$ vivant soit présent chez le fondateur $j$ appartenant à sa lignée est :

$$
\mathrm{W}_{i j}=\left(\mathrm{S}_{i j / k}\right)(1 / 2) n
$$

$n$ est le nombre de générations séparant $i$ de $j$ et la somme se fait sur tous les chemins $k$ allant de $i$ à $j$.

Pour l'ensemble d'une population, on additionne les paramètres $\mathrm{W}_{i j}$ et on obtient la part du génome provenant du fondateur $j$. Une étude menée sur un isolat Dogon du Mali (Cazes, 1986) a montré que la population, bien qu'endogame à $85 \%$, ne possédait finalement que $42 \%$ de gènes originaires des fondateurs de l'isolat. Ceci montre la différence pouvant exister entre les niveaux d'endogamie et de consanguinité. 


\section{Chapitre II}

\section{L'ANTHROPOLOGIE DÉMOGRAPHIQUE}

\section{I. - Le champ de l'anthropologie démographique}

L'unité familiale, selon l'expression de Françoise Héritier, «sert à faire des enfants pour reproduire la société ». Le remplacement des générations est un aspect majeur de la structure et de l'évolution des populations humaines. Les comportements reproductifs sont influencés par un ensemble de paramètres dont il est difficile de décrire le poids respectif, tant les interactions sont complexes. La question de la reproduction ne peut pas s'analyser indépendamment du contexte de la mortalité, ni de celui du milieu aussi bien écologique, social que culturel. Phénomène biologique, la reproduction humaine est aussi l'objet de stratégies. La dimension de la famille, qui en est le résultat comptable, prouve par son extrême diversité selon les pays et les groupes humains qu'il s'agit bien là d'un phénomène du plus grand intérêt pour l'anthropologue.

1. La famille. - Il existe des modèles familiaux qui diffèrent selon les cultures et qui évoluent en fonction des conditions de l'environnement économique des sociétés. D'ailleurs, nombreuses sont les disciplines qui ont pris pour cadre et objet d'étude la famille.

La famille, évoluant selon le temps, les situations 
et les contextes, il est important d'avoir des images de sa structure ou de sa composition à un moment donné. Mais il est aussi nécessaire, pour analyser un phénomène dans sa dynamique, d'accoler les instantanés successifs pour dérouler le film de la vie des individus qui la composent. En ce sens, historiens, démographes et anthropologues se retrouvent pour accorder une grande importance à la notion de cycle de vie. Les historiens, à la suite des travaux de Peter Laslett (1972) sur la structure du ménage dans l'Europe ancienne, et les démographes avec le développement des approches méthodologiques des biographies familiales ont largement repris à leur compte cette notion de cycle de vie. Il s'agit d'une notion que les anthropologues, pour leur part, avaient déjà largement fait leur, en situant leurs travaux dans la perspective de la succession des générations et la transmission des patrimoines (Augustins, 1989).

Bien d'autres facteurs sont encore à considérer pour réaliser une étude pertinente de la famille. A part la question du cycle de vie, l'étude de la famille doit aussi s'effectuer en tenant compte du contexte économique global de la société et du milieu de vie des populations (par ex., rural/urbain ou milieux écologiques très contrastés). Il ne faut pas non plus négliger l'univers familial, à la fois cadre de la vie quotidienne, lieu d'exercice de la parenté, de l'entraide autant que des conflits entre les générations, des rapports entre les sexes. La famille a aussi, à part sa fonction universelle et essentielle de reproduction, celle d'être une unité de consommation, de production ou d'échange. Variable selon les cultures, les lieux et les époques, la famille est une institution en constante évolution qui est souvent le reflet des transformations qui affectent la société globale.

2. La reproduction. - Le terme de reproduction (entendu au sens de reproduction biologique des indi- 
vidus et des groupes) correspond à une action dont la finalité est le remplacement des générations. Il s'agit là d'un phénomène essentiel pour apprécier la structure et l'évolution des populations humaines et c'est à ce titre qu'il intéresse les anthropologues.

Ainsi, sur des populations de pays industrialisés dans les années 1950, Penrose (1959) montre que 100 grossesses n'aboutissent qu'à seulement 76 individus en âge de procréer, et pouvaient à leur tour participer au processus de la continuité de l'espèce humaine. En effet, sur les 100 grossesses initiales, certaines n'arrivent pas à terme, il y a des enfants mortsnés et parmi les enfants nés vivants certains viennent à mourir avant d'atteindre l'âge de la procréation. Parmi ces 76 personnes, environ $20 \%$ ne se marient pas et $10 \%$ n'ont pas d'enfants. Sur 100 grossesses, seulement 56 personnes auront un descendant. On comprend avec cet exemple la complexité du phénomène de reproduction dont la variabilité biologique est largement tributaire de conditions de milieu et de celui des fondements culturels. En ce sens, la démographie nous servira dans ce cás à mesurer ces phénomènes populationnels et l'anthropologie à expliquer les variations selon les cultures.

La notion de reproduction recouvre également une grande diversité de situations. Elle est très liée aux questions de santé, à tel point que l'on parle maintenant de santé reproductive en ce qui concerne tout ce qui comprend les modalités de gestion par la mère de sa grossesse jusqu'à la naissance de l'enfant. Cette tendance correspond à une attitude plus générale constatée aujourd'hui dans les sociétés occidentales tout autant que dans les pays du Tiers Monde, où l'on assiste à une forte médicalisation de phénomènes biologiques. Pour la grossesse, on constate que la prise en charge médicale dépend largement du niveau d'éducation de la mère, de même que l'on trouve une corrélation entre le niveau de scolarisation des fem- 
mes et le taux de mortalité infantile dans les pays en développement. Un autre exemple est celui de la ménopause, processus biologique ancré dans le social, qui devient de plus en plus un objet du discours médical.

Sauvain-Dugerdil (1996) parle même d'abandonner l'emploi du terme de reproduction, qui lui semble trop connoté comme un phénomène se rattachant à la permanence des choses et ne prenant pas suffisamment en compte les aspects de changement. Elle propose d'utiliser le terme de procréation si l'on raisonne au niveau individuel et celui d'évolution si l'on se situe au niveau du groupe.

3. Le concept de génération. - Chez les anthropologues, les notions de génération et d'âge ont été très discutées. En effet, dans les sociétés traditionnelles, ces notions sont en rapport étroit avec l'organisation sociale des groupes étudiés. Tous les auteurs se reconnaissent dans la distinction entre la classe ou groupe d'âge (ensemble de personnes ayant a peu près le même âge) et celle d'échelon d'âge (grandes subdivisions de la vie). Ces subdivisions sont cependant très théoriques et la réalité montre une infinité de variations qui met en évidence la complexité de cette question (Attias-Donfut, 1988).

L'appréciation des modalités et des rythmes d'évolution d'une population s'effectue à partir de l'étude de la succession des générations. Ce choix différencie les anthropobiologistes des démographes, car pour les premiers, la population de référence est celle qui participe au processus de reproduction, excluant alors les célibataires et les couples infertiles. Les terrains de recherche peuvent être très divers et prendre des formes spatio-temporelles différentes selon les populations étudiées : étude des migrations temporaires, de ceux qui partent et de ceux qui restent par la reconstitution des généalogies et des structures des 
ménages, étude de la relation population/ressources et de la transition démographique par la mesure de la mortalité infantile et de la fécondité, ou encore étude des modalités d'insertion de populations migrantes par l'appréciation des comportements matrimoniaux.

Il existe trois définitions de la génération: acte d'engendrer, degrés de filiation successifs, appartenance à une même classe d'âge. Le concept de génération est utilisé dans de nombreuses disciplines, mais dans des sens parfois fort différents. Nous ne retiendrons que les deux dernières définitions précédemment énoncées et qui correspondent à celles usitées respectivement par les anthropologues et les démographes. Chez ces derniers, avec le phénomène d'allongement de la durée de la vie et le rôle différent assigné aux personnes du troisième âge dans nos sociétés, le concept de génération devrait prendre une place plus importante au détriment de la notion d'âge. Il existe depuis quelques temps un emploi plus fréquent du concept de génération chez les sociologues, en particulier depuis les études sur la mobilité sociale et sur l'émigration. Ce sont les sociologues américains qui ont réactualisé l'emploi du concept de "génération » (au sens de filiation) dans leur discipline en avançant l'idée, maintenant très répandue dans bien d'autres domaines du champ social, qu'il faut trois générations à une famille d'immigrée pour s'adapter dans son pays d'accueil. De la même façon, - il est intéressant de noter que la plupart des sociologues actuels travaillant sur la famille tendent également à intégrer dans leurs analyses des approches anthropologiques (Segalen, 1981). Cet ensemble de faits nous permet de considérer l'emploi du concept de génération comme pertinent dans les études d'anthropologie démographique.

Parmi la multitude des situations, deux exemples qui nous semblent révélateurs de l'importance de la notion de génération: 
- Les conséquences de l'allongement de la durée de la vie dans les pays occidentaux.

A défaut de la cohabitation des générations sous le même toit, surtout en milieu rural et principalement chez les agriculteurs, il y a de nouveaux rapports qui s'établissent. Les enfants d'agriculteurs font souvent construire une maison qui se situe la plupart du temps à proximité de celle de leurs parents, au sein même de l'exploitation agricole. Même en ville, où les modes de vie et d'habitat obligent à la prédominance de la famille nucléaire, il existe malgré tout des relations très importantes entre les parents et les enfants. Et même dans le cas où les parents et leurs enfants ont des résidences géographiquement éloignées, des liens assez forts demeurent souvent et prennent alors la forme d'aide financière apportée aux jeunes ménages ou de vacances estivales des petits-enfants chez leurs grands-parents.

C'est ainsi que de nouvelles relations s'établissent entre générations, phénomène qui renforce l'idée de racine, de transmission familiale, du poids ou de «l'empreinte de la lignée ». On assiste donc à une tendance relativement récente dans la société moderne d'un retour à des aspects traditionnels dans les relations familiales entre les générations.

- Les conséquences de la migration de populations issues de sociétés traditionnelles vers les pays industrialisés.

Nous avons souvent affaire à une migration individuelle qui fait place ensuite à un regroupement familial, soit par la venue de l'épouse restée au pays, soit par un mariage du migrant avec une conjointe épousée au pays lors d'un retour pour une période de vacances. Dans l'immense majorité des cas, on note l'absence des grands-parents et des collatéraux dans la famille reconstituée en France. Il s'agit alors d'une "parenté mutilée » lorsque nous avons à faire à des migrants africains. Il en découle bien souvent des dif- 
fïcultés dans l'éducation des enfants et une perturbation dans l'image du rôle des parents. Il y a une rupture dans la succession des générations, et donc passage d'une société traditionnelle à des comportements et des organisations du groupe familial différents. La tendance générale est à l'affaiblissement du poids des générations comme le faisait remarquer Mead (1971) à propos de la rupture entre le passé (ascendants connus) et le présent (parents familiers).

Ces deux exemples sont le reflet de situations qui expriment des évolutions inverses. Dans le cas des familles occidentales il y a renforcement des relations intergénérationnelles. Dans les familles migrantes, à l'inverse, il y a un affaiblissement du lien générationnel. Ces changements ne peuvent cependant pas être encore appréciés dans leur pleine dimension compte tenu de leur apparition récente.

Il existe aussi des limites dans l'emploi du concept de "génération 》. En théorie, chacun appartient à une génération, mais en pratique il existe des mariages entre apparentés. Différentes méthodes sont employées pour reconstruire la population (transversale associée à l'âge, longitudinale basée sur les généalogies). Mais quel que soit le choix de la méthode, il y a toujours une grande part d'arbitraire. D'autant que le recueil oral des généalogies ne permet pas non plus d'atteindre une totale fiabilité (Cazes, 1987).

En démographie, les cohortes de naissance, définies comme des générations, sont difficiles à exploiter dans l'étude des populations migrantes. Certains éléments viennent perturber les analyses classiques, en particulier l'ancienneté de la migration (première génération) et les modalités de la constitution de la famille. Alors que dans d'autres cas, comme dans la migration de retour au pays des Portugais émigrés en France, l'utilisation d'une cohorte de deux générations se prête bien à l'analyse et permet d'effectuer 
une comparaison riche en enseignements. Chaque génération se distingue de l'autre par son cycle de vie, le contexte historique et son expérience migratoire différente. Le retour au Portugal des immigrés de première génération est celui de ruraux qui achètent de la terre qu'ils n'avaient pas en partant, le retour des deuxièmes générations, avec la plupart du temps l'acquisition de commerces, correspondant à un choix de vie différent (Acheson, 1993).

L'anthropologie démographique se situe donc à la charnière des préoccupations des anthropologues qui privilégient les liens familiaux et s'intéressent aux nombreuses facettes de la parenté et de la transmission des patrimoines et de celles des démographes qui observent plutôt les dynamiques familiales, le mode de constitution de la famille, le mariage et la fécondité.

\section{II. - Les méthodes utilisées \\ dans les recherches d'anthropologie démographique}

En démographie, les méthodes servent à décrire et analyser la structure et la dynamique de populations humaines dans l'espace et dans le temps. Par exemple, compter et classer, c'est tenter d'évaluer l'effectif (et donc la densité) de la population et son évolution (natalité, nuptialité, mortalité). Il faut aussi envisager les modalités et les rythmes de la dynamique de cette même population en examinant son aptitude à se reproduire et à s'adapter à de nouvelles conditions (exemple des travaux sur les mobilités). Le plus souvent la mesure de phénomènes implique une nécessaire quantification pouvant aller jusqu'à la construction de modèles hautement sophistiqués.

Toutes ces méthodes sont inégalement employées dans les recherches anthropologiques et parfois même contre-indiquées. Dans le cas de la reproduction d'une population, des facteurs démographiques, 
sociaux et biologiques interagissent. Par exemple, l'intensité du célibat, qui est un paramètre important à considérer dans une recherche démographique, ne présentera pas le même intérêt pour une étude d'anthropologie démographique dans la mesure où ce sont des personnes qui ne participent pas au renouvellement des générations.

\section{La notion d'observatoire et le fichier de population.}

- En permettant l'étude d'une population de façon continue dans le temps, l'observatoire de population est un outil très utile pour l'anthropologue qui veut décrire et comprendre les processus du changement. Un observatoire de population exige l'articulation entre plusieurs types d'enquêtes qui doivent se compléter et correspondre à l'observation d'un même processus temporel. Dans la mesure où nous avons à faire à l'étude de plusieurs populations d'effectif important, avec une grande quantité d'information, il est nécessaire de constituer, sous forme informatisée, ce qu'il est convenu d'appeler un «fichier de population ». II permet de structurer l'information, et de produire des analyses. On peut ainsi dégager des tendances, effectuer des comparaisons diachroniques, étudier la structure et la dynamique d'un phénomène particulier. Deux conditions doivent être réunies pour que le fichier de population soit un bon outil d'analyse : la détermination d'une séquence temporelle d'étude et le choix d'indicateurs pertinents pour apprécier le processus étudié. Enfin, s'agissant de gros fichiers informatisés (grand nombre d'individus et de variables), il faut aussi être conscient des limites liées à la masse critique de l'information ou de la question éthique et juridique posée par l'exploitation de renseignements individuels d'ordre privé.

Ces fichiers se construisent facilement pour des populations de faible effectif dont on souhaite étudier l'évolution sur une longue période. C'est le cas des 
monographies de démographie historique réalisées en France sur de petites communautés villageoises au cours des siècles précédents. Il peut aussi s'agir de l'étude d'une fraction ou d'un échantillon de population, comme par exemple l'enquête «des trois mille familles » pour apprécier la mobilité en France au cours des deux derniers siècles. A l'inverse on peut citer des fichiers qui concernent des populations d'effectifs importants dont les objectifs de recherche sont alors différents. Deux exemples sont impressionnants par la dimension de la population étudiée. Celui des travaux des chercheurs de Salt Lake City qui ont pour objectif de constituer une gigantesque base de données (Utah Mormon Genealogy) pour répertorier toute la population ascendante des Mormon et qui comprendrait plus de huit millions de fiches. L'autre étude concerne la région du Saguenay, au Québec, étudiée par une équipe pluridisciplinaire composée d'historiens, de démographes, d'anthropologues et de généticiens (Bouchard, 1990). L'objectif est d'élucider la dynamique d'une population depuis ses origines au XVII 'siècle jusqu'à nos jours, en procédant à la reconstitution généalogique des familles à partir des données de l'état civil. L'originalité consistait en la création d'une banque de données avec une gestion de fichiers très performante. L'ambition n'était pas de se limiter aux seules données démographiques, mais de faire une étude d'histoire totale en accolant aux données démographiques des informations économiques, sociales, culturelles, médicales. Cette étude, un peu comme celle sur la population des Mormons, trouve d'ailleurs sa motivation essentielle dans une recherche d'identité.

L'utilisation $\mathrm{du}$ fichier de population présente aussi l'intérêt de pouvoir effectuer des comparaisons de l'évolution d'un processus particulier dans différentes populations. On peut prendre comme exemple le phénomène de la migration (diversité des popula- 
tions migrantes, des lieux géographiques et des époques) où, pour que le fichier de population reste un outil d'analyse efficace, il peut être judicieux de choisir une séquence temporelle d'étude équivalente à trois générations. Le fichier de population et son traitement résolument quantifié ne sont que le plus petit dénominateur commun de ce type de travail et l'approche qualitative et la prise en compte du local ne doivent pas être négligées. D'autant que l'approche comparative présente des limites. Il est parfois difficile d'obtenir une homogénéité des informations recueillies, condition pourtant essentielle pour l'application de la méthode comparative. En effet, les populations étudiées n'ont pas toutes la même valeur informative dans la mesure où la qualité de celle-ci est dépendante de l'unité géographique de référence et les rythmes d'évolution différents éventuellement constatés peuvent provenir d'un effet d'échelle.

2. L'état civil et la méthode généalogique. - Dans une étude anthropologique destinée à décrire et expliquer la structure et la dynamique d'une petite population rurale au cours d'une période temporelle qui s'étale sur plusieurs générations, l'exploitation des informations contenues dans les actes de mariage de l'état civil est indispensable. On peut en effet apprécier l'évolution de l'âge au mariage, de l'endogamie/ exogamie de naissance et résidence, de l'homogamie socioprofessionnelle...

Les limites méthodologiques de telles études permettent de constater que seules les personnes mariées sur place sont prises en considération. D'autre part, il n'est pas possible de mesurer l'intensité du célibat, dont on connaît pourtant le rôle régulateur qu'il joue dans l'évolution des communautés rurales occidentales. Malgré ces restrictions, l'analyse des actes de mariages reste tout de même intéressante. 
La construction d'une généalogie est un travail long et fastidieux, puisqu'il faut généralement effectuer des recherches à travers les registres d'état civil de différentes localités, à cause de la mobilité des personnes. Il faut tout d'abord commencer par chercher les ancêtres d'un couple actuel (généalogie ascendante) et après avoir retrouvé les fondateurs de la lignée ou avoir couvert la période temporelle choisie pour l'étude, retrouver toutes les personnes qui sont issues des couples fondateurs (généalogie descendante). En pratique, les étapes à franchir s'organisent ainsi : à partir des actes de naissances des individus qui forment le couple actuel, on relève sur les actes les mentions marginales du mariage (ou des divorces et mariages successifs éventuels), comportant les dates, lieux et nom du conjoint, pour les périodes récentes. On note également la date et lieu de décès s'il y a lieu. Puis on se reporte à l'acte de mariage pour connaître les données concernant les parents des mariés, en particulier leur dates et lieu de naissance, pour ensuite renouveler la même opération et passer à la génération précédente. Cette méthode permet de reconstituer une généalogie ascendante. Si l'on souhaite obtenir une profondeur généalogique importante, la recherche se complexifie, car sur les actes de naissances, les mentions marginales de mariage figurent à partir de 1870 et celles de décès ne sont notées qu'après la guerre de 1945.

La reconstitution de généalogies descendantes est moins aisée, car il faut repérer la période où chaque couple est susceptible d'avoir eu des enfants et chercher les événements possibles dans le registre des naissances, sachant que les couples ont pu être mobiles et avoir eu des naissances ailleurs que dans leur commune de mariage ou de résidence actuelle. Un exemple classique, dans les campagnes françaises, avec la tendance à la résidence virilocale (la future épouse vient habiter dans la commune de son mari). 
Dans ce cas, il n'est pas rare, lors de l'accouchement du premier enfant, que la femme retourne dans sa famille. Il arrive alors que l'enfant soit déclaré dans la commune de résidence des grands-parents maternels. Si l'habitude est pourtant de faire des transcriptions, ce qui correspond à l'obligation pour la commune où s'est passé l'événement de déclarer à la commune de résidence des parents la naissance, cette réglementation n'a pas toujours été bien appliquée et il y a de nombreuses omissions.

On comprend bien que cette méthode est très différente de la méthode de reconstitution des familles de Louis Henry laquelle, pour une même unité territoriale, généralement une paroisse ou une commune, saisissait toutes les naissances, mariages et décès survenus dans l'unité étudiée sur une période de temps déterminée. Cette méthode permet d'étudier une population dans son cadre administratif, sans prendre en compte la mobilité des individus, ce qui, même dans des populations rurales considérées comme peu mobiles, n'était pas satisfaisant pour apprécier la réalité à la fois des structures familiales (on peut être né dans en lieu et ne pas y résider, ou le contraire) ni celle des lignées.

\section{La liste nominative de recensement et la structure} des ménages. - Pour effectuer une étude biodémographique d'une population française, un deuxième document indispensable à utiliser est la liste nominative de recensement. Soulignons que l'exploitation de cette source nous paraît autant appropriée pour apprécier les modalités de reproduction d'une population rurale sédentaire que dans le cas de la dynamique d'un processus migratoire.

Le recensement est effectué en France par période quinquennale. Les listes sont constituées aux mêmes dates que les recensements par le personnel de mairie des communes. Celle de 1836 est la première du 
genre, au plan national. Les individus sont répertoriés, en fonction d'un classement par ménage.

La méthode de recueil de l'information par ménage semble la plus appropriée, car elle permet de ne pas exclure les célibataires, de mieux saisir les mobilités individuelles, et d'effectuer une étude plus pertinente des structures et comportements familiaux. Elle permet surtout d'opérer un jumelage des listes et offre ainsi la possibilité d'une étude longitudinale de la population. On peut alors quantifier la mobilité générale au cours d'une période intercensitaire (naissances, décès, départs et arrivées d'individus isolés et de ménages). On peut également conduire une étude plus détaillée des émigrants et immigrants selon le sexe, l'âge, la profession, la situation familiale, le lieux de résidence, et comparer chacun des deux sous-groupes entre eux et avec la population restée sédentaire. Seules sont comptabilisées dans la liste, les personnes domiciliées dans la commune et qui y vivent habituellement, qu'elles soient présentes ou non au moment du recensement. Les renseignements portés sur les listes sont dans l'ordre le lieu de résidence, puis le numéro de l'individu et celui de son ménage, ses nom et prénoms, sa profession, son sexe et son état matrimonial (célibataire, marié, veuf), son âge et enfin une colonne observation qui peut selon le cas préciser la parenté au sein du ménage ou bien le statut professionnel (patron ou employé/ouvrier). D'une façon générale, les données portées sur les listes sont plutôt faciles à recueillir. $\mathrm{La}$ seule véritable difficulté, dans quelques cas peu nombreux, est de bien repérer les liens de parenté entre les différents membres composant un même ménage. L'examen des patronymes et des âges permet d'y remédier dans la presque totalité des cas.

Pour conduire une analyse qui soit à la fois la plus riche en information et qui corresponde à la meilleure photographie de la réalité économique et sociale d'une population, le plus efficace est le recueil des 
données en prenant comme unité d'étude le ménage, repéré par sa résidence et le patronyme de son chef. Il suffit ensuite de relever pour chacune des personnes constitutives de ce ménage, l'âge, le sexe, l'état matrimonial et le lien de parenté. Cette façon de procéder permet ainsi l'étude de la totalité des ménages de la population, en utilisant par exemple la typologie mise au point par Peter Laslett (1972) dont nous expliquerons l'intérêt dans la partie consacrée aux analyses, et offre une possibilité de pouvoir effectuer des comparaisons avec d'autres populations. Il y a cependant des situations où le choix du ménage (défini par l'INSEE comme toutes les personnes qui vivent sous un même toit) n'est pas le plus adapté aux conditions de l'étude. Des recherches effectuées auprès des immigrés maghrébins dans la ville de Marseille montrent qu'il est difficile de dénombrer avec précision les personnes qui constituent le ménage. La définition habituelle du ménage ne correspond pas toujours au mode de vie d'un ménage migrant. Une personne non domiciliée peut y prendre la totalité de ses repas ou bien certains enfants peuvent être déclarés résidents sans y dormir, et cela sans compter les parents de passage que l'on héberge parfois pendant de longues périodes. Dans ce cas, le ménage immigré ne peut donc pas être uniquement considéré comme une unité de résidence, mais plutôt comme un foyer de consommation inclus dans un réseau d'interconnaissances.

4. L'enquête biodémographique dans les pays en développement. - Les problématiques et les analyses restent les mêmes, mais les méthodes diffërent du fait que ces pays ne possèdent généralement pas d'état civil dont les données soient fiables, ni de recensements nationaux réguliers et suffisamment précis. Les démographes réalisent souvent en complément des enquêtes locales, surtout sur des populations urbaines (le monde rural est souvent méconnu), ou bien plus spécifiques et 
générales comme les enquêtes mondiales de fécondité (WFS) ou les enquêtes démographie et santé (EDS).

La seule façon d'obtenir des données de qualité est donc de passer par le moyen d'enquêtes de terrains directement auprès des populations. C'est ce que fait l'anthropologue qui cherche à reproduire le schéma d'analyse adopté dans les pays où les sources sont fiables. Il établit une fiche afin de chiffrer le nombre total de personnes vivant dans le ménage et leur position respective. Il cherche à connaître, pour chaque membre du ménage, l'identité, l'âge, le lieu de naissance ainsi que les liens de parenté existant entre les personnes qui composent cette unité de résidence. La difficulté réside dans la détermination précise des liens de parenté, surtout lorsqu'il y a plusieurs épouses. Quel est le statut des enfants du ménage, et quels sont leurs parents biologiques. Une autre difficulté sera, dans des populations parfois très mobiles, d'évaluer la fréquence des déplacements et leur durée (sauf en cas de très longues absences).

Il y a aussi la question de la fiabilité du recueil des données auprès des intéressés eux-mêmes. Une recherche destinée à mesurer l'évolution de la fécondité et de la mortalité dans une région du Maroc permet d'illustrer cet aspect (Crognier, 1987). Les enquêtes avaient été conçues pour réunir, en plus des résultats démographiques et biologiques, des paramètres descriptifs de l'environnement familial des femmes. Un soin particulier avait été porté à la collecte de l'information. Les infirmiers itinérants des différents secteurs de la province avaient été mobilisés comme enquêteurs, étant considérés comme le personnel le plus qualifié pour collecter des informations concernant la mortalité, puisque le plus sensibilisé à ces questions, en même temps qu'il avait aussi une bonne connaissance de la population et se trouvait reconnu par elle. Pourtant, l'examen des conditions d'enquête avaient mis en évidence la difficulté 
d'aborder la mort des enfants, le problème de l'omission (volontaire ou non) de certains décès et l'imprécision dans la datation des événements.

\section{5. Échelle d'étude et homogénéité de la population.} - Il s'agit de deux paramètres dont l'emploi, dans les études de démographie anthropologique, présente souvent quelques difficultés.

Un article introductif (Fassin, 1996) à un récent numéro de la revue Prévenir sur le thème «Ville et santé », défend l'idée qu'il faut aujourd'hui choisir le local comme espace d'analyse et comme échelle d'observation. Il considère qu'une approche du local n'empêche pas un certain degré de globalisation, et favorise une analyse plus fine qui peut faire apparaître des différences qui seraient indécelables dans des études à plus grande échelle. En cela, il n'est pas très éloigné du propos de Jacques Gomila (1977) qui montre qu'une des approches restant l'une des moins critiquables est celle qui retient comme unité d'étude la notion de petite communauté, définie le plus souvent comme une population locale en étroite relation avec l'écosystème naturel. Autrement dit, il s'agit alors d'une population de petite taille, montrant des caractères d'homogénéité et présentant une autarcie économique.

L'autre difficulté concerne l'homogénéité de la population et la place de l'individu dans le groupe. Pour la résoudre, démographes et sociologues utilisent l'analyse biographique qui associe le plus souvent des données individuelles et longitudinales. L'idée est que les comportements individuels ne peuvent pas être dissociés d'un contexte familial, social et culturel.

Cette approche pose en effet la question du rapport individu/groupe. Y a-t-il plutôt pouvoir du groupe sur les individus ou bien au contraire autonomie des individus au sein du groupe? Le groupe familial joue en effet un rôle important dans les trajectoires individuel- 
les. Des études de sociologie qui étudient la mobilité sociale ou d'anthropologie qui étudient la transmission des biens ou des pouvoirs sont là pour le montrer (Augustins, 1989). Souvent, la proximité de résidence de l'individu et de ses proches semble un bon estimateur de l'intensité du lien qui existe. Cette constatation avait déjà été formulée par des anthropologues qui avaient montré, dans un quartier londonien, que les liens des enfants avec leurs parents n'étaient pas aussi lâches que le laisserait supposer un mode de vie urbain, centré sur l'unité familiale et l'individualisme ambiant généralisé. Il semblait que des liens assez forts persistaient entre la fille et sa mère, et qu'ils s'expliquaient souvent par des domiciles rapprochés qui permettaient ainsi que des rapports étroits puissent perdurer. Les paramètres importants sont la résidence et les liens d'alliance. On peut toutefois s'interroger si la composition du groupe dépend uniquement de l'impact conjugué de la corésidence et de la parenté. Le groupe peut s'élargir par exemple aux liens d'amitié et dans ce cas la notion d'entourage semble plus opératoire et plus proche de la réalité que le concept de famille, trop flou ou celui de ménage, trop restrictif (Lelievre, 1997).

6. L'apport des méthodes qualitatives. - Pour l'étude d'une population, deux ensembles de méthodes apparemment opposées, mais en réalité complémentaires, se présentent : ce sont les enquêtes par questionnaires et les méthodes qualitatives. A chacune ses avantages, ses inconvénients et sa place dans l'organisation des recherches. Observations et entretiens permettent de décrire et d'apprécier les comportements d'une façon fine et détaillée pouvant par la suite servir de support à l'élaboration d'un questionnaire mettant en évidence certains points particuliers valant la peine d'être approfondis. L'observation participante consiste, au fil de la vie quotidienne, à mener 
une approche de l'intérieur et de l'extérieur tout en corrigeant le contenu des discours obtenus par entretiens individuels ou collectifs. Les entretiens collectifs avec des groupes cibles homogènes permet d'explorer les attitudes, croyances, opinions, préférences, valeurs et comportements d'une population. Leur principal avantage provient de la dynamique d'interaction entre les participants. La récolte de récits de vie semble bien convenir pour apprécier l'évolution au cours du temps de certains comportements.

La migration est un bon exemple de syncrétisme des méthodes employées, celles qui font appel à l'exploitation des sources administratives (recensements, archives d'état civil), et celles qui concernent l'histoire transmise oralement au travers de générations successives de migrants. Elle rejoint ainsi une double approche consistant à concevoir la migration à la fois comme un phénomène statistique qui s'inscrit comme un facteur de modification de l'espace, de l'économie et de la démographie, mais aussi comme un ensemble de destins individuels. Tout le problème de l'articulation entre cas « représentatifs » et cas «marginaux », c'est-à-dire de la quantification du qualitatif, se trouve dépassé si on les introduit dans un ensemble. Le problème posé est de voir comment il est possible d'effectuer une écriture de l'histoire d'un phénomène social à partir de l'articulation entre des documents administratifs porteurs d'informations factuelles et des entretiens avec des acteurs plus ou moins proches du processus historique que l'on veut cerner.

\section{III. - Les principales analyses en anthropologie démographique}

1. Structures familiales, formation et composition du ménage. - La plupart du temps, les études des structures familiales se basent sur une classification des 
types de ménages établie par Peter Laslett (1972). Le terme de ménage désigne dans ce cas l'appartenance à un lieu d'habitation et implique donc une communauté de vie. Il y a six types qui vont par ordre croissant de complexité et d'effectif : ménage solitaire (personne seule veuve ou célibataire), ménage sans structure familiale (corésidents apparentés ou non), ménage simple ou communément appelé famille nucléaire (couple avec enfants éventuels), famille élargie (famille nucléaire augmentée de membres apparentés), ménage multiple (groupement de plusieurs familles apparentées), ménage à structure indéterminée. La fréquence des différents types permet de discuter des modes d'organisation selon les époques et les sociétés, en particulier pour le rapport entre le pourcentage de familles nucléaires et de ménages multiples.

Les systèmes familiaux sont différents selon les régions françaises et ils influent sur la dynamique démographique des populations. Par exemple, dans le sud-est de la France, région plutôt considérée comme un pays de famille-souche, l'habitude fut toujours de doter les filles, en les excluant alors de l'héritage, et de privilégier un héritier parmi les garçons. Ce fonctionnement impliquait qu'il fallait marier rapidement les filles, de façon à faire rentrer dans la maison une bru qui deviendrait la conjointe de l'héritier, les autres garçons apprenant par ailleurs un métier pour laisser la place à celui à qui revenait la ferme. Il s'agit d'un système inégalitaire et autoritaire, puisque c'est le chef de ménage qui désigne son héritier et surtout qui conserve la direction de l'exploitation tant qu'il le juge nécessaire. Ce système présente souvent quelques dysfonctionnements qui ne sont pas sans conséquence démographique, notamment sur l'âge au mariage, dont on note une augmentation dès la fin $\mathrm{du} \mathrm{XVIII}^{\mathrm{e}}$ siècle en Haute-Provence. Une autre conséquence est aussi le remariage fréquent chez les veufs. Dans la zone méditerranéenne, la règle en matière de mariage est large- 
ment conditionnée par l'économique. Dans le Niolo (en Corse), où la propriété familiale est faible et où il existe une forte proportion de communaux, une famille possédant de nombreux fils pourra ainsi avoir un troupeau plus important, puisque plus de bergers pour le garder. Cette conséquence sur la dimension de la famille n'est pas la seule, car pour conserver le patrimoine communal, voire même de hameau dans certains cas, il faut exclure les étrangers et par conséquent favoriser un type de mariage endogame.

2. La mortalité. - - La mortalité est un élément fondamental pour l'évolution des groupes humains. Les problématiques anthropologiques envisagent, en fonction des niveaux et modes de vie des populations, leurs diversités de comportements en matière de santé. La formule déjà ancienne d'un démographe disant que "l'ignorance tue plus que la pauvreté ", autrement dit, l'ignorance et la façon de vivre qui l'accompagne, est encore d'actualité. Le paramètre aujourd'hui essentiel est l'espérance de vie, dont on observe l'augmentation constante à la fois dans les pays industrialisés et ceux en développement.

Les démographes ont l'habitude de partager la vie humaine en deux grandes périodes s'étendant pour la première de la conception à l'âge d'un an, et pour la seconde jusqu'à la mort. Cette division n'est pas arbitraire, car la mortalité de la première année, remarquable par son importance en nombre, apparaît comme très liée à la mortalité totale. La mesure du niveau de mortalité des enfants n'est toutefois pas suffisant pour évaluer le niveau de développement d'une population; il faut aussi établir la liste des déterminants de la mortalité et tenter d'en apprécier l'impact. Ce sont les déterminants socioculturels de la mortalité en général et de celle des enfants en particulier qui constituent sans doute l'ensemble de facteurs le plus étudié et le mieux connu. Citons par exemple 
la dimension de la fratrie, le rang de naissance, la résidence urbaine ou rurale, les revenus du ménage, l'instruction de la mère, la catégorie socioprofessionnelle du père, le type d'allaitement, les modalités de sevrage, les vaccinations. Il ne faut pas non plus négliger les déterminants géographiques et écologiques, même si leur impact sur la mortalité est souvent plus difficile à évaluer et encore sujet à des controverses qui découlent d'un ancien débat sur le déterminisme du milieu. Parmi eux, le climat peut occuper une place prépondérante.

Étant donné qu'il existe tout un ensemble de facteurs qui régissent le niveau et la structure de la mortalité infantile, plusieurs auteurs ont tenté d'élaborer des modèles de déterminants de la mortalité des enfants. L'un des plus récent (Barbieri), reprend celui déjà établi par Mosley et Chen. Il envisage deux grands ensembles de déterminants (ceux qui appartiennent à l'environnement écologique et les déterminants socio-économiques, culturels, politiques) qui conditionnent l'exposition au risque de maladie. C'est de cette exposition au risque - selon le degré de résistance des populations, leur niveau de morbidité, l'efficacité des thérapies mises en œuvre - que dépendra en dernier ressort l'intensité de la mortalité. Le système de santé, dépendant des déterminants socioéconomiques, intervient dans ce schéma au plan de la résistance des populations (infrastructures existantes et politiques de prévention) et des actions thérapeutiques développées pour les personnes malades. Il existe continuellement des tentatives pour élaborer de nouveaux modèles, et le dernier en date prend en compte le cadre historico-géographique, politicoéconomique, communautaire et familial, sanitaire (Tabutin, 1997).

Les anthropologues s'intéressent aux populations des pays en développement lesquelles, engagées dans un processus d'urbanisation et de modernisation sont 
en pleine transition démographique, et présentent partout une diminution de leur mortalité générale. S'agissant de la mortalité infantile (c'est-à-dire des décès des enfants de moins de un an), la méthode du démographe $J$. Bourgeois-Pichat permet de séparer les décès infantiles en deux grandes catégories: les décès dits « endogènes » et les décès dits " exogènes ». Les décès «endogènes 》 sont définis comme le prolongement de la mortalité prénatale et résultent de causes antérieures à la naissance ou de la naissance elle-même. La mortalité endogène provient de la mauvaise constitution de l'embryon, d'une mauvaise santé de la mère pendant la grossesse, ou bien encore de difficultés liées à l'accouchement. La mortalité " exogène 》 comprend l'ensemble des décès imputables au milieu dans lequel vit l'enfant. Les causes exogènes les plus courantes sont les infections d'origines diverses (essentiellement digestives et respiratoires), les maladies épidémiques, les accidents et les malnutritions.

Distincts par leurs causes, les décès «endogènes » et « exogènes » se différencient également par l'âge auquel ils frappent les jeunes enfants. Les décès endogènes sont concentrés sur le premier mois et les décès exogènes s'étalent entre le premier et le douzième mois. On s'aperçoit qu'il existe une surmortalité exogène, c'est-à-dire une mortalité exceptionnelle due à des conditions de milieu très défavorables qui dépendent de facteurs économiques, sociologiques, sanitaires et écologiques. Dans les zones à climat chaud la 《 surmortalité exogène » débute entre trois et six mois et on l'attribue essentiellement aux infections d'origine alimentaire. D'ailleurs, dans les pays où la loi de Bourgeois-Pichat se trouve vérifiée les infections alimentaires arrivent toujours dans le groupe de tête des causes de décès. Mais le climat agit aussi directement sur les infections du système respiratoire. Dans une étude sur la mortalité en Algérie, si les 
maladies diarrhéiques arrivent en tête des causes de mortalité infantile, les maladies de l'appareil respiratoire arrivent en seconde position. En définitive, c'est l'amélioration des conditions sanitaires, de l'hygiène et de la couverture médicale tout au long de la deuxième moitié du XIX ${ }^{\mathrm{e}}$ siècle jusqu'à la seconde guerre mondiale, qui aura un impact sur les décès exogènes.

On s'aperçoit aussi que la surmortalité exogène est plus importante chez les filles que chez les garçons. Le phénomène de surmortalité féminine se retrouve d'ailleurs en France jusqu'en 1930. L'explication de la surmortalité féminine est délicate. On sait en effet, que le rapport de masculinité est toujours légèrement supérieur à l'unité (il naît un peu plus de garçons que de filles), mais cet avantage numérique des garçons se trouve ensuite compensé par des décès plus nombreux (imputables à une plus grande fragilité biologique). La surmortalité féminine proviendrait plutôt de comportements culturels. La conjugaison d'un ensemble de facteurs irait dans le sens d'une moindre attention portée aux filles, les exposant ainsi à un risque plus important d'agressions du milieu et en définitive à une probabilité plus élevée de décéder que les garçons.

3. La fécondité. - Paramètre fondamental du renouvellement d'une population et située à l'interface du biologique et du culturel, la fécondité est au centre des analyses en anthropologie démographique. En démographie, cette question est souvent abordée à grande échelle et nécessite la construction d'indices pour caractériser l'évolution d'une population. Ce n'est d'ailleurs pas chose simple, comme le montre le débat qui s'est instauré en 1990 entre démographes français sur la question de savoir si la population française était en dessous ou au-dessus de son seuil de renouvellement. Cette polémique portait sur l'utilisation de deux méthodes différentes pour calculer le 
nombre moyen d'enfants par famille : celle qui utilisait l'indice conjoncturel de fécondité trouvait 1,8 enfant par femme et celle qui se référait à la descendance finale arrivait à 2,1. Cette discussion. ( $L a$ Recherche, 1990) aura eu l'avantage de mettre en lumière la difficulté et la complexité d'un phénomène fortement ancré dans le social et lié aux perceptions du monde par chacun de nous.

Pour les anthropologues, la question des indices et de l'évolution des effectifs de population se pose moins que de comprendre les facteurs qui déterminent les comportements en matière de fécondité et les variations et interactions d'un processus biologique inscrit dans le culturel.

La base de départ est bien biologique, c'est la fertilité ou aptitude à concevoir pour une femme, que Louis Henry a appelé fécondité naturelle (c'est-à-dire sans limitation volontaire des naissances). Différents facteurs conditionnent l'intensité de ce phénomène : la durée de la vie féconde (entre l'âge d'apparition des premières règles et la ménopause), la durée du temps mort (gestation et aménorrhée postpartum), l'intensité des décès intra-utérins et enfin les stérilités partielles ou totales.

Toutefois, tous ces facteurs sont aussi dépendants d'éléments culturels. Par exemple, la variabilité de l'âge d'apparition des premières règles, mis à part des facteurs génétiques selon les populations, est en relation avec le niveau de vie, l'alimentation, l'univers familial, la résidence... Même si l'on s'achemine vers une homogénéisation de niveaux et modes de vie, des différences notables existaient pourtant dans les pays industrialisés il y a encore de cela une ou deux décennies, où plusieurs études montraient que les filles de milieux économiques favorisés et résidant en milieu urbain présentaient un âge d'apparition des premières règles inférieur d'un an aux autres filles. Pour la ménopause, les études actuelles sont encore insuffi- 
samment développées pour apprécier les raisons des différences possibles. A cela, ajoutons que la durée de vie féconde se résume parfois à une durée de vie matrimoniale, le nombre des naissances hors mariage restant encore très minoritaire, et que cette durée dépend de l'âge au mariage qui est extrêmement fluctuant selon les sociétés. De la même façon, la durée de l'aménorrhée post-partum dépend en partie de l'alimentation et présente une forte liaison avec l'allaitement, dont on sait bien que sa pratique et sa durée sont très liées aux effets de mode ou de celui du discours médical, souvent très changeant dans nos sociétés, ou encore aux comportements traditionnels dans certains pays en développement. On pense en particulier à l'interdit de relations sexuelles durant l'allaitement afin que le sperme ne nuise pas à la qualité du lait et que cela ne soit pas préjudiciable à la santé de l'enfant. Enfin, les stérilités partielles sont souvent la cause, comme en Afrique de l'Ouest, de maladies sexuellement transmissibles telles que la syphilis. Il s'agit d'une maladie qui dépend des comportements adoptés par les populations en matière de prévention et de gestion de la sexualité. Les travaux actuels des sociologues de la santé sur le sida confirment bien l'impact des comportements individuels en matière d'épidémie comme de maladie transmissible d'une façon générale.

Au-delà de ces aspects sociaux du déroulement d'une fonction biologique, il y a l'action des individus sur la fécondité, qui se traduit par un acte volontaire de contrôle ou de limitation des naissances par l'emploi de méthodes contraceptives. L'un des indicateurs indirect pour mesurer l'impact des méthodes contraceptives est l'intervalle entre naissances, pour lesquels on fait la différence entre l'intervalle protogénésique, qui s'étend du mariage à la première naissance et les intervalles intergénésiques qui concernent les espaces entre les naissances suivantes s'il y en a. 
Cette séparation s'explique par le fait que l'intervalle protogénésique varie peu selon les sociétés et les époques, les couples récemment mariés n'attendant généralement pas pour avoir un premier enfant. En revanche, les intervalles suivants sont l'objet de variations parfois importantes qui sont révélatrices de comportements différents des familles. Nous en donnerons des exemples dans la troisième partie qui étudiera quelques populations ou situations particulières.

4. La migration. - Les processus migratoires présentent un caractère universel, quelque soit l'époque, le lieu ou la population étudiée et ils ont joué un rôle fondamental dans l'évolution et l'histoire de l'humanité. Quand l'homme change de milieu (c'est-àdire lorsqu'il effectue une migration), il lui faut en effet s'adapter à de nouvelles conditions de vie.

A) La migration et ses aspects génétiques et démographiques. - S'agissant de petits groupes, la question du changement génétique opéré par la migration s'envisage selon quelques grands critères : la distance et le rôle éventuel de barrières, le mariage et l'exogamie, la direction préférentielle dans l'espace des déplacements humains. Le rôle de la distance apparaît comme majeur. Mais la mesure de la corrélation entre la distance et les différences de fréquences géniques dépend de l'irrégularité de la distribution géographique et surtout de données démographiques telles que la densité de l'effectif des populations concernées.

La figure 2 montre la relation qui existe entre densité, taille des groupes et pourcentage d'endogamie. Elle compare, en fonction de ces trois paramètres la distribution de la distance entre les lieux de naissance des conjoints, pour trois populations vivant dans des conditions très différentes.

La représentation de la distance entre lieux de nais- 

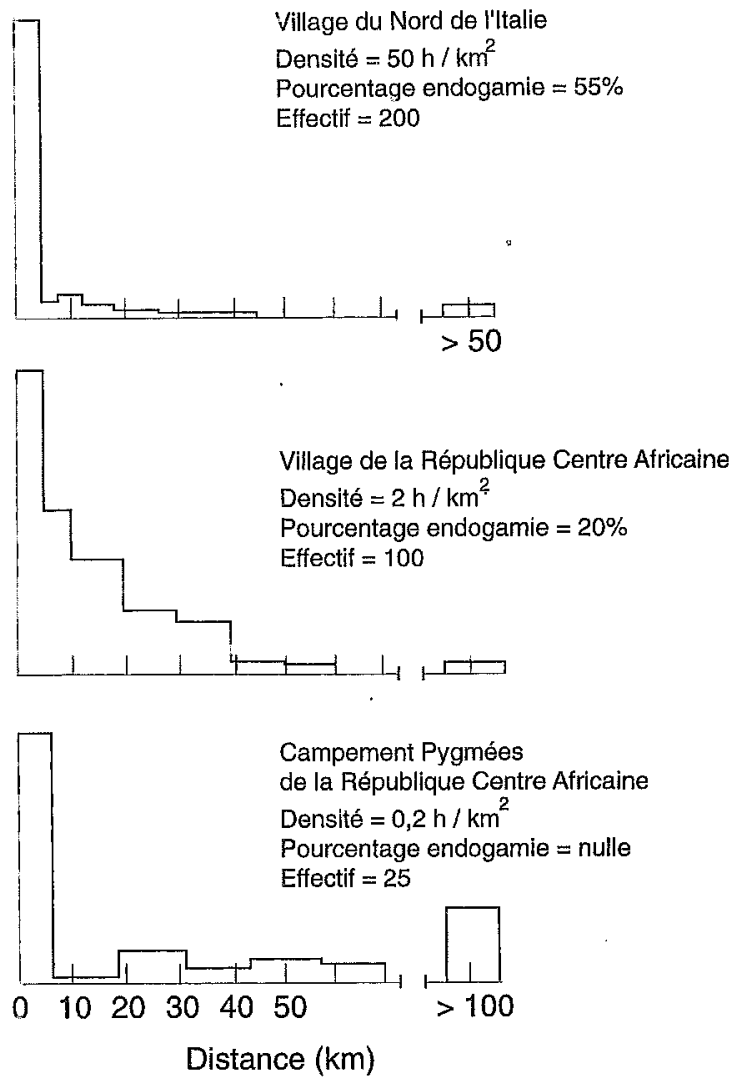

Fig. 2. - Comparaison de la distribution des distances entre le lieu de naissance des époux pour trois populations (d'après Bodmer et Cavalli-Sforza, 1968). 
sances des conjoints montre que, sur une distance inférieure à $50 \mathrm{~km}$, celle-ci diminue de façon exponentielle pour les villageois européens et africains (avec une décroissance plus nette chez les Européens), alors qu'elle est indépendante de la distance chez les Pygmées. On s'aperçoit aussi qu'il existe une relation entre l'effectif de population et l'endogamie, une population qui présente un effectif suffisant est plus endogame qu'une population de plus faible effectif.

Pour affiner cette analyse, Bodmer et Cavalli-Sforza ont mis en relation, dans des villages de montagne du nord de l'Italie, l'effectif de ceux-ci avec le pourcentage de personnes immigrées dans ces mêmes villages. Ils mettent en évidence que ces deux paramètres varient de façon inversement proportionnelle, c'est-àdire que les villages de faible effectif accueillent de nombreux immigrés dont le nombre diminue lorsque les effectifs des villages croissent. Comme il s'agit en grande partie de migrations matrimoniales et en particulier de femmes qui viennent habiter au domicile du mari, on en déduira qu'une population de petite taille est contrainte de chercher des conjoints à l'extérieur alors que cette obligation est moindre lorsque l'effectif total est plus élevé. L'analyse des migrations matrimoniales aura de nombreux développements et sera très utilisé en anthropologie. Plusieurs problématiques seront abordées. Certains tenteront de comprendre la relation existant entre consanguinité, exogamie et distance matrimoniale. D'autres s'attacheront plutôt à décrire les modalités de la migration matrimoniale selon la distance, dont la traduction graphique sera élaborée pour la première fois par Kucheman (1967) à partir de données recueillies sur des paroisses de l'Oxfordshire en Angleterre.

B) Définir le mouvement des migrants. — Pour définir le mouvement, la méthode la plus usitée est celle qui emploie les matrices de migration, à condition 
que l'on admette que les migrations soient constantes à l'intérieur des périodes temporelles d'étude. Cela se vérifie généralement assez bien pour des populations historiques. Le reste du temps, la méthode n'est pas très fiable et les flux migratoires sont affectés par la croissance démographique, la proximité d'autres centres et la diversification économique. Les matrices sont des tableaux de nombres à partir desquels on effectue des opérations. Les nombres sont appelés des éléments et s'organisent en un tableau rectangulaire où ils sont rangés en ligne et en colonne. Certaines matrices possèdent des caractéristiques particulières. C'est le cas des matrices dites stochastiques qui se distinguent par deux aspects : les éléments qui les composent sont toujours positifs ou nuls, et leur somme en ligne est égale à 1 . Ce type de matrice est très utile pour apprécier des phénomènes comme le processus migratoire, où un élément $a i / j$ de la matrice représente la probabilité de passer d'un état $(i)$ à un autre $(j)$, les éléments situés sur la diagonale de la matrice (a $i / i)$ représentant alors la probabilité de ne pas changer d'état.

Un très intéressant et très simple exemple d'utilisation de cette technique peut se trouver dans le travail de recherche de Harvey et Suter (1984) sur les îles Feroé. Appartenant au Danemark et situées face à la Norvège, au nord de l'Écosse, elles sont formées de reliefs abrupts et de caps farouches, exposées aux vents violents et souvent noyées dans les brumes. L'isolement a contribué à préserver les traditions de ces populations insulaires et à y maintenir des habitudes de solidarité. Les conditions naturelles défavorables à une extension des activités agricoles n'ont laissé aux habitants que la possibilité de vivre à partir des seules ressources de la mer.

Les auteurs ont voulu mesurer le degré de mobilité des populations entre ces îles et apprécier l'évolution de ce phénomène dans le temps (cf. fig. 3). Pour 


\begin{tabular}{|c|c|c|c|c|c|c|c|c|c|}
\hline \multirow{2}{*}{$\begin{array}{c}\text { Lleu } \\
\text { de naissance } \\
\text { des enfants }\end{array}$} & \multicolumn{9}{|c|}{ Lieu de naissance des parents } \\
\hline & 1 & 2 & 3 & 4 & 5 & 6 & 7 & Etranger & Total \\
\hline 1 & 0.811 & 0.115 & 0.014 & 0.008 & 0.012 & 0.020 & 0.011 & 0.009 & 1.0 \\
\hline 2 & 0.025 & 0.889 & 0.036 & 0.009 & 0.008 & 0.013 & 0.011 & 0.009 & 1.0 \\
\hline 3 & 0.007 & 0.069 & 0.789 & 0.056 & 0.014 & 0.019 & 0.032 & 0.014 & 1.0 \\
\hline 4 & 0.008 & 0.060 & 0.013 & 0.858 & 0.018 & 0.023 & 0.020 & 0.000 & 1.0 \\
\hline $\mathbf{5}$ & 0.015 & 0.037 & 0.033 & 0.013 & 0.807 & 0.040 & 0.047 & 0.008 & 1.0 \\
\hline 6 & 0.010 & $0.04 B$ & 0.011 & 0.022 & 0.043 & 0.824 & 0.027 & 0.015 & 1.0 \\
\hline 7 & 0.060 & 0.109 & 0.105 & 0.030 & 0.056 & 0.072 & 0.553 & 0.015 & 1.0 \\
\hline
\end{tabular}

\begin{tabular}{|c|c|c|c|c|c|c|c|c|c|}
\hline \multirow{2}{*}{$\begin{array}{c}\text { Lieu } \\
\text { de naissance } \\
\text { des enfants }\end{array}$} & \multicolumn{9}{|c|}{ Lieu de naissance des parents } \\
\hline & 1 & 2 & 3 & 4 & 5 & 6 & 7 & Etranger & Total \\
\hline 1 & 0.728 & 0.149 & 0.014 & 0.028 & 0.028 & 0.039 & 0.011 & 0.003 & 1.0 \\
\hline 2 & 0.031 & 0.862 & 0.031 & 0,008 & 0.012 & 0.081 & 0.017 & 0.008 & 1.0 \\
\hline 3 & 0.019 & 0.128 & 0.670 & 0,044 & 0,022 & 0.049 & 0.057 & 0.011 & 1.0 \\
\hline 4 & 0.030 & 0.059 & 0.023 & 0.786 & 0.006 & 0.030 & 0.023 & 0.043 & 1.0 \\
\hline 5 & 0.010 & 0.062 & 0.036 & 0.031 & 0.736 & 0.073 & 0.036 & 0.016 & 1.0 \\
\hline 6 & 0.017 & 0.049 & 0.026 & 0.019 & 0.024 & 0.820 & 0.024 & 0.021 & 1.0 \\
\hline 7 & 0.049 & 0.272 & 0.136 & 0.070 & 0.066 & 0.111 & 0.288 & 0.008 & 1.0 \\
\hline
\end{tabular}

Fig. 3. - Matrices de migration pour les régions des îles Faroe, selon le lieu de naissance parents/enfants pour les deux générations successives

(d'après Harvey et Suter, 1984). 
atteindre cet objectif, ils ont interrogés la totalité des enfants scolarisés (soit 1398 personnes) nés une même année (1960) dans toutes les îles pour connaître leur lieu de naissance, celui de leurs parents et de leurs grands-parents. Ils ont ensuite organisés ces données en des matrices stochastiques en croisant le lieu de naissance d'une génération et celui de ses parents pour chacune des îles. Les données ont été ramenées à des fréquences. Pour chaque île on peut voir quelle est la contribution des autres en pourcentage; par exemple si l'on examine les résultats d'une ligne, on notera que sur la totalité des enfants nés dans l'île $1,81,1 \%$ des parents y sont aussi nés, puis $11,5 \%$ de parents sont nés dans l'île 2, $4 \%$ dans l'île 3 , etc., et si l'on somme toutes les îles on arrive à un total de $100 \%$. Cette organisation des données en une matrice stochastique est très suggestive à l'œil et permet de bien repérer quelles sont les îles attractives et quelles sont celles dont la population émigre. On notera que la diagonale de la matrice est bien l'illustration qu'il n'y a pas de changement d'état, c'est-à-dire dans le cas qui nous intéresse que les lieux. de naissance des enfants et des parents sont identiques. Ce n'est d'ailleurs pas un hasard si c'est sur cette diagonale que l'on retrouve les plus fortes valeurs qui correspondent à l'absence de migration examinée à travers les lieux de naissances de deux générations. Cela indique que la règle générale est la sédentarité d'une génération à l'autre et que la migration d'une île à l'autre reste limitée. L'analyse des données montre une évolution entre les deux périodes et une mobilité accrue dans la période plus récente. Elle montre aussi que certaines îles ont des populations peu mobiles et qu'à l'inverse d'autres (et en particulier celle dans laquelle se trouve la ville et le port important) sont très attractives et reçoivent de nombreuses personnes originaires des îles avoisinantes. 


\section{Chapitre III}

\section{QUELQUES EXEMPLES}

\section{I. - Les populations de chasseurs-cueilleurs}

$\mathrm{Ce}$ qui a profondément marqué l'histoire de l'humanité, c'est la rupture démographique qui s'est réalisée dans beaucoup de régions du monde au moment de « la révolution néolithique ». Pourtant, il existe encore de nos jours, de très rares sociétés humaines dont le mode de vie et les structures sociales nous semblent relever d'une situation antérieure à l'économie néolithique. Ce sont les sociétés de chasseurs-cueilleurs. Il s'agit de groupes humains dont l'activité économique repose sur la chasse, la pêche, le ramassage et la collecte.

Leur étude est d'un intérêt primordial pour nous aider à analyser la dynamique des relations entre populations et ressources, car le nombre de paramètres à prendre en compte chez les chasseurs-cueilleurs est beaucoup plus réduit que dans le cas de sociétés complexes comme la nôtre.

\section{Environnement et population.}

A) Écosystèmes et densité de la population. - Si les contraintes du milieu ne peuvent expliquer à elles seules la structure démographique des groupes de chasseurs-cueilieurs, car nous savons aujourd'hui que les 
facteurs sociaux contribuent aussi au maintien de l'équilibre démographique, elles interviennent fortement comme facteurs de régulation face à l'accroissement potentiel de la population. Des anthropologues anglo-saxons ont montré que les facteurs écologiques influençaient très fortement la dimension d'un groupe puisqu'elle dépend des ressources en nourriture et en eau mis à sa disposition.

Les connaissances démographiques que nous possédons par l'intermédiaire de recensements de population sont très variables d'un groupe à l'autre. En ce qui concerne la dimension moyenne des groupes de chasseurs-cueilleurs, on peut retenir deux chiffres: celui de 35 personnes pour le campement (c'est-à-dire l'unité de production économique) et celui de 500 personnes pour la bande (c'est-à-dire l'unité d'échanges sociaux).

$\mathrm{Si}$, quelque soit la nature de l'écosystème, la dimension et la structure du groupe par âge et par sexe sont assez comparables entre populations de chasseurs-cueilleurs, par contre, l'étendue du territoire occupé, exprimée sous la forme de densité de population, variera fortement selon les milieux.

Chez les Pygmées Bayaka, en zone tropicale, on observe la présence d'une trentaine de personnes par campement et la densité est de 28 personnes/100 $\mathrm{km}^{2}$. A titre de comparaison, la densité de la population française est de $91 / \mathrm{km}^{2}$ et pour l'Inde ou la Chine, on obtient des valeurs régionales pouvant atteindre $400 / \mathrm{km}^{2}$. Chez les Jivaros Shuar qui pratiquent largement 1'horticulture, et bien que résidant en forêt tropicale, on note des densités moins importantes $\left(17 / 100 \mathrm{~km}^{2}\right)$.

Lorsque l'on observe les chiffres concernant les régions désertiques, on trouve des densités encore plus faibles. Ainsi, pour les déserts chauds (comme celui du Kalahari) on constate que les Dobe! Kung ont un effectif moyen de 25 personnes par groupe et une den- 
sité d'environ 15 personnes/100 $\mathrm{km}^{2}$ (Lee, 1968). Chez les aborigènes d'Australie, les densités paraissent encore bien plus faibles et peuvent descendre jusqu'à 2 personnes $/ 100 \mathrm{~km}^{2}$.

Pour les déserts froids, comme ceux de la région des grands lacs canadiens, les densités sur les territoires de chasse sont assez semblables. Il y a, par exemple, 9 personnes $/ 100 \mathrm{~km}^{2}$ chez les Algonkians (Hallowell, 1949). Dans l'ensemble, la densité de population est 4 fois plus faible en milieu désertique qu'en milieu tropical.

Ainsi, les facteurs climatiques interviennent de façon incontestable dans l'équilibre démographique. Nous savons que l'eau est un des éléments essentiel dans la chaîne trophique. En Australie, la superficie des territoires tribaux est inversement proportionnelle à la quantité de pluie restant en surface après évaporation (Tindale, 1953). Ceci a été confirmé par Birdsell (1953) qui a trouvé une corrélation positive de .80 entre la densité de population et la hauteur des précipitations pour 123 tribus australiennes. L'espace nécessaire à un groupe de chasseurscueilleurs, pour qu'il puisse assurer sa subsistance et sa survie, est un problème important. Mais celle de se procurer de l'eau est une contrainte impérative. Les ressources du milieu naturel sont des facteurs limitant pour l'expansion du groupe (en particulier, impossibilité technologique de transporter de l'eau) alors que la défense d'un territoire ou du groupe implique un nombre minimal de chasseurs et/ou de guerriers.

$\mathrm{Au}$ total, trois principaux facteurs se conjuguent afin de maintenir une faible densité démographique : les ressources potentielles, les capacités du groupe à se procurer ces ressources et le niveau de consommation. La relation entre ces trois facteurs a été énoncée par Hassan (1975) dans la formule suivante :

$$
\mathrm{P}_{d}=f(\mathrm{R}, \mathrm{E}) / \mathrm{L}
$$


dans laquelle $\mathbf{P}_{d}$ est la densité de population, $\mathrm{R}$ correspond aux ressources potentielles (en gros la productivité primaire nette de l'écosystème), E représente les capacités des membres du groupe à obtenir l'énergie nécessaire à la survie du groupe et $\mathrm{L}$ son niveau de consommation.

$\mathrm{La}$ variable $\mathrm{E}$ exprime le bilan énergétique du groupe. Ainsi, dans le cas de l'activité cynégétique, il vaut mieux tuer un gros gibier qu'un petit pour compenser la dépense énergétique occasionnée, ou encore pratiquer la chasse collective qui offre une meilleure stratégie alimentaire que la chasse individuelle. Cet indice $\mathrm{E}=\mathrm{O} / \mathrm{I}$ peut aussi se définir comme un indice de capacité technique pour acquérir des ressources $(\mathrm{O}=$ outputs, c'est-à-dire l'énergie que procure le système au groupe humain; I = inputs, c'est-à-dire l'énergie que le groupe doit dépenser pour se procurer des ressources). Des études comparées ont montré que E variait de 9,6 chez les Kung à 11,2 chez les agriculteurs de Gambie et qu'il atteint 53,5 chez les riziculteurs chinois. Ces résultats montrent la disponibilité énergétique importante que procure l'agriculture par rapport à la chasse et à la cueillette, permettant ainsi aux populations agricoles d'accroître leur niveau de fécondité grâce à l'augmentation de leurs ressources alimentaires disponibles (dans 1'hypothèse où l'on souscrit à la théorie malthusienne). Mais cette capacité des ressources alimentaires suffisantes dépend aussi de la structure démographique du groupe. Wrigley (1976) a calculé que les producteurs devaient représenter $56 \%$ du groupe pour assurer un équilibre démographique interne. Condition qui va modeler le profil de la pyramide des âges de la population (sous forme d'un tronc) avec une forte représentation des classes d'âge actives.

Le niveau de consommation du groupe (L) dépend de l'écosystème et de la structure démographique du groupe. Il est directement associé à la 
dimension du groupe et aux ressources dont il peut disposer sur le territoire qu'il occupe. Celles-ci doivent d'ailleurs se situer dans un rayon de $10 \mathrm{~km}$ autour du campement car au-delà de cette distance, l'énergie dépensée n'est plus couverte par les gains obtenus. Cette nécessité de demeurer à moins d'un jour de marche des réserves de chasse et de collecte est directement dépendante des saisons. Dans les zones semi-désertique - comme la région du $\mathrm{Ka}$ lahari où vivent les Bushmen - l'activité est rythmée par un cycle annuel qui fait généralement se succéder trois types de situations: la période de novembre à avril durant laquelle les points d'eau sont nombreux et les aliments abondants, celle de mai à juillet correspondant au début de la saison sèche avec des aliments encore abondants mais où la vie se concentre autour des points d'eau permanents et enfin une période d'août à octobre avec des ressources alimentaires et des points d'eau de plus en plus rares.

B) Les contraintes de l'écosystème sur l'équilibre démographique.

a) Les ressources alimentaires. L'état nutritionnel est important puisqu'il constitue un des facteurs jouant sur la physiologie de la reproduction. Les grands types d'écosystèmes au sein desquels vivent les chasseurs-cueilleurs vont déterminer la spécificité de leurs activités économiques (pêche, chasse, cueillette...) qui vont elles-mêmes répondre à leurs besoins alimentaires.

Ainsi, et bien que le milieu leur offre une diversité alimentaire relativement élevée, on constate que les chasseurs-cueilleurs ont une action limitée sur celui-ci. Par exemple, les Pygmées Aka ne consomment guère plus d'une centaine de plantes sur les 3000 espèces qui existent au sein de leur environnement et ne prélèvent qu'une douzaine de vertébrés sur 600 . Il est vrai que les Pygmées représentent un cas particulier dans 
la mesure où ils vivent dans un écosystème extrêmement riche et diversifié, chose assez rare chez les chasseurs-cueilleurs. D'ailleurs, cette richesse ne demeure pas la propriété de quelques-uns, mais est redistribuée à l'ensemble du groupe. Des règles sociales, comme les tabous alimentaires, réduisent les inégalités dans l'accès aux aliments. Ainsi, les chasseurs ne doivent pas consommer la viande de leur propre chasse mais la redistribuer aux autres membres du groupe (Bahuchet, 1979). Toutes les populations de chasseurscueilleurs adoptent des tabous alimentaires; mais ce sont essentiellement les femmes qui en sont frappées lors de trois événements physiologiques qui leur sont spécifiques: les règles (premières règles et règles menstruelles), la grossesse et l'allaitement.

Dans d'autres écosystèmes pourtant moins̀ diversifiés, l'écart entre espèces présentes et espèces consommées reste cependant important. Ainsi, chez les Kung, 85 végétaux sur 200 et 19 espèces animales sur 220 sont consommés.

b) Les régimes alimentaires. L'activité de 6 Bushmen durant cinq jours par semaine permet de nourrir un groupe de 10 personnes, et les rations alimentaires couvrent les besoins des individus. A titre d'exemple, la ration alimentaire des Kung est constitué par $37 \%$ de viande (690 cal.), $33 \%$ de noix Mongongo (1 290 cal.) et $30 \%$ d'autres végétaux (190 cal.), dont les melons et les concombres qui leur procurent de l'eau en période sèche, soit $2170 \mathrm{cal}$. (Lee, 1969). Ce régime alimentaire semble adapté aux besoins car leur métabolisme de base (repos) nécessite $1400 \mathrm{cal} . / \mathrm{j}$ pour les hommes et $1100 \mathrm{cal} / \mathrm{j}$ pour les femmes. Comme les hommes mesurent $1,57 \mathrm{~m}$ en moyenne pour un poids de $46 \mathrm{~kg}$ et les femmes $1,47 \mathrm{~m}$ pour un poids de $41 \mathrm{~kg}$, on s'aperçoit que leurs besoins journaliers en calories (2 000) sont couverts. Par contre, en période d'intense activité - comme la chasse - l'équilibre semble plus difficile à atteindre. Sachant que les besoins énergéti- 
ques s'élèvent à $200 \mathrm{cal} . / \mathrm{h}$ de marche et que la chasse nécessite souvent huit heures de marche par jour, les besoins supplémentaires s'élèvent à 1600 cal. (sans tenir compte des variabilités interindividuelles). Le régime alimentaire des Kung montre que, contrairement à ce qui se passe chez les Pygmées, ce n'est pas la diversité des espèces qui leur garantit une alimentation riche, mais l'importance quantitative d'une d'entre elles en particulier, la noix Mongongo. D'après Sahlins, des dizaines de milliers de noix sont ramassées, ce qui ne représente pourtant que la moitié des noix disponibles.

Dans certains écosystèmes comme celui des Aches, situé dans l'est du Paraguay, certaines périodes de l'année peuvent fournir des surplus caloriques impressionnants (Hill, 1984). En effet, dans leur écosystème forestier, les Aches s'approvisionnent quotidiennement en gibier et la quantité consommée est très régulière. Ils consomment peu de végétaux, mais par contre, ils sont sur-nutris en miel entre les mois de décembre et février (leur consommation calorique atteint alors $5500 \mathrm{cal}$. en novembre).

Enfin, on a pu constater que les habitudes alimentaires des chasseurs-cueilleurs étaient très sélectives; le choix des espèces s'effectue selon quatre critères d'importance décroissante : le goût, la valeur nutritive reconnue, l'abondance et enfin la facilité pour se les procurer.

c) L'impact de l'environnement sur la mortalité. Le facteur alimentaire est prépondérant dans la survie des groupes humains. En règle générale, la mortalité due à la malnutrition est un phénomène rare chez les chasseurs-cueilleurs, contrairement à ce que l'on observe dans les populations d'agriculteurs-éleveurs (au Sahel, par ex.). Généralement, chez les chasseurscueilleurs, la pénurie alimentaire n'entraîne pas de grave crise démographique, mais c'est un facteur qui limite souvent la croissance démographique en inhi- 
bant la fertilité. D'autres facteurs - comme la guerre, les accidents ou les épidémies - contrarient l'accroissement de la taille des populations de chasseurscueilleurs. L'espérance de vie à la naissance est peu élevée et de ce fait les maladies de dégénérescence sont peu fréquentes. Ainsi, très peu de cancers ou de maladies cardio-vasculaires ont été trouvés dans les populations de chasseurs-cueilleurs que l'on a étudiées ( $2 \%$ des décès).

II existe bien sûr des différences selon le niveau d'acculturation. On observe chez les Esquimaux une augmentation des cancers du poumon suite à l'apparition de l'usage du tabac. Le même phénomène s'observe pour le diabète. Les décès dus aux maladies cardio-vasculaires, associés à une absence d'hypertension, sont rares. La relation milieu/maladie est relativement évidente dans ce cas: les populations vivant en milieu tropical humide ont une faible consommation de sodium. Dans le milieu polaire, la consommation y est beaucoup plus élevée ainsi que la pression artérielle. Pourtant les Esquimaux ne présentent pas de surmortalité due aux complications cardio-vasculaires, prouvant ainsi la variabilité physiologique des populations.

En revanche, les accidents constituent généralement une part importante des décès chez les chasseurscueilleurs encore que de notables différences apparaissent selon les écosystèmes. Dans les régions polaires, les principales causes de décès par accidents sont : les noyades, brûlures, suffocations et accidents de chasse. Entre 1900 et 1960 , les décès par maladie ont régressé de 77 à $47 \%$ alors que les décès par accidents sont passés de 19 à $29 \%$. Dans les régions tropicales, les décès par accidents sont plus rares, et l'adaptation physiologique au climat est bien meilleure.

En résumé, trois principaux facteurs protègent les chasseurs-cueilleurs contre les maladies épidémiques : l'isolement, la faible dimension de la population et sa 
mobilité. Dans une étude portant sur les Indiens Jivaros, on a observé que les parasites intestinaux et les maladies épidémiques (comme la fièvre jaune) étaient assez répandus dans cette population pourtant relativement isolée. Dans ce cas, l'explication est à rechercher dans les anciens contacts des Jivaros avec des populations d'esclaves africains qui les auraient introduites. Ces épidémies affaiblissent les populations et peuvent provoquer des maladies de malnutrition. La mobilité est un facteur efficace de protection en empêchant la propagation des agents infectieux ayant besoin d'un long cycle de latence. C'est pourquoi les Indiens Yanomami se divisent en petits groupes familiaux durant les épidémies et restent isolés les uns des autres selon le principe de la quarantaine. Cette faible densité de population va freiner considérablement la propagation transversaie des foyers d'infection, mais elle n'empêchera pas la propagation verticale entre générations.

En revanche, les maladies infectieuses de la peau ne sont pas rares chez les chasseurs-cueilleurs. Le premier homme blanc qui est entré en contact avec les Aborigène Australiens, en 1829, avait noté que de nombreux individus étaient vérolés. Ce type de maladie n'est pas sans conséquences sur les structures biologiques et sociales des populations. On a observé que les femmes qui en sont atteintes se marient plus tardivement que les autres, et qu'elles sont souvent les secondes ou troisièmes épouses dans les sociétés polygames. Les unions tardives donnant moins d'enfants, ii s'agit donc d'un facteur sélectif.

Ce phénomène sélectif n'est évidement pas le seul au sein des populations de chasseurs-cueilleurs. Si l'on prend par exemple le problème de la vision, que ce soit celui de l'acuité visuelle ou encore celui de la dyschromatopsie (anomalies de la vision des couleurs), on voit que les facteurs sélectifs prennent toutes leur importance. L'acuité visuelle est assez déter- 
minante dans les groupes de chasseurs et de pêcheurs et on a remarqué que les populations africaines et arctiques avaient une bien meilleure vision que les populations européennes. Par contre, le daltonisme est un handicap pour les cueilleurs où l'effet sélectif risque d'être plus rapide : une erreur dans la cueillette de baies peut entraîner une élimination rapide de l'individu et donc de ses gènes au sein de la population. Le taux de daltonisme dans les populations de chasseurs-cueilleurs est en moyenne de $2 \%$, alors qu'il est de $8 \%$ dans les populations européennes. Chez les Esquimaux d'Alaska, on a trouvé seulement $1 \%$ de daltoniens parmi les individus non métissés alors que les enfants issus d'unions mixtes présentent un taux de daltonisme de $6,5 \%$.

2. Les paramètres démographiques dans les populations de chasseurs-cueilleurs. - Les populations de chasseurs-cueilleurs adoptent-elles des comportements malthusiens ? Pour parvenir à un équilibre, les populations disposent de deux principaux facteurs régulateurs : un contrôle de la natalité ou une élimination d'une partie de la population. Les réponses des populations humaines, y compris celles des chasseurscueilleurs vont dépendre de facteurs culturels, euxmêmes influencés par les contraintes du milieu. De ce fait, il existe une grande variabilité de situations entre populations.

A) Les caractéristiques démographiques.

a) La fécondité. Les études effectuées sur les Bushmen Kung ont montré comment l'alimentation pouvait influencer la fécondité. En effet, on a pu constater que les conceptions étaient minimales en juin et en août, périodes où les apports caloriques et protéiques sont aussi les plus faibles (Wilmsen, 1979).

D'autre part, sachant que l'allaitement nécessite 1000 calories supplémentaires par jour en plus de 
l'énergie nécessaire au déplacement, un déficit calorique durant trois ou quatre années consécutives peut faire baisser le niveau de fécondité (Howell, 1979). L'âge auquel survient les règles est un facteur physiologique qui influence la fécondité. Ce facteur dépend des conditions de milieu en général et de l'alimentation en particulier. A partir d'une étude effectuée sur un échantillon d'environ 150 filles et sur une période de dix ans, Howell a montré que l'âge des premières règles des jeunes filles Kung se situerait dans une tranche d'âge comprise entre 15 et 17 ans et la première naissance entre 18 et 22 ans. Mais ce facteur semble dépendre autant de facteurs génétiques qu'environnementaux puisque chez certaines tribus d'Aborigènes australiens, les Yanomami ou les Esquimaux d'Asie ont constate un âge aux premières règles plus précoce.

b) L'intervalle intergénésique. L'espacement des naissances est un autre facteur conditionnant la fécondité des femmes. Dans une population qui n'utilise pas de moyens contraceptifs modernes, ce paramètre va fortement influencer la descendance finale des couples. Dans l'ensemble, les espaces intergénésiques sont élevés chez les chasseurs-cueilleurs. Chez les Pygmées Bayaka, la lactation dure entre deux et trois ans, et les naissances ont lieu tous les quatre ans. Pour les Kung, des données plus précises sont fournies par Howell concernant un échantillon de femmes ménopausées, donc en fin de reproduction :

$\begin{array}{ll}\text { Intervalle protogénésique } & 3,9 \text { ans } \\ \text { Dernier intervalle } & 4,6 \text { ans } \\ \text { Intervalle moyen } & 4,1 \text { ans }\end{array}$

Une femme ne peut pas être enceinte tant que son dernier-né n'est pas capable de marcher seul pour des déplacements qui peuvent être assez longs (soit vers l'âge de 4 ans). Chez les Yanomami, moins mobiles, l'intervalle génésique moyen est plus bas; 3,4 ans 
(Chagnon, 1974). Dans les populations de chasseurscueilleurs, un espacement des naissances d'au moins trois ans semble une contrainte démographique imposée par le mode de production.

En plus des facteurs biologiques, certains groupes mettent en cuvre des contraintes sociales pour réduire la natalité. Chez les Asmat de NouvelleGuinée, il existe un tabou des relations sexuelles pendant les deux années qui suivent la naissance d'un enfant (Townsend, 1980). Chez les Djuka de Nouvelle-Guyane, les règles d'abstinence postpartum sont très strictes (cité par Wrigley, 1969); il s'agit d'une méthode fréquemment employée pour limiter les naissances. Cette abstinence sexuelle est performante parce que régie par de nombreux tabous. Chez les Jivaros, la régulation des naissances s'appuie sur des tabous de relations sexuelles qui apparaissent lors de toute une série de pratiques sociales: guerre, chasse, chamanisme, construction de canoë... On y rencontre aussi l'utilisation de plantes contraceptives. Enfin, les maladies comme la malaria ou la tuberculose se révèlent aussi être un facteur d'abaissement du niveau de fertilité en accroissant l'intervalle intergénésique ou en provoquant la stérilité (M.-C. Elroy, 1989).

c) La mortalité infantile. Chez les Kung, l'intervalle intergénésique assez élevé ( 3 à 5 ans) correspond à un nombre de 8 naissances pour les femmes ayant achevé leur vie féconde. La mortalité infantile est élevée puisque seuls 4,7 enfants survivent.

Deux sortes de facteurs vont généralement influencer le taux de mortalité infantile, ceux liés à l'action du milieu (comme les maladies) et les facteurs sociaux (tels que les conditions d'accouchement, l'infanticide...). La mortalité infantile totale atteint $200 \%$ (entre 0 et $1 \mathrm{an}$ ), et la mortalité juvénile $140 \%$ (entre 1 et 5 ans). Chez les Esquimaux Ammassalimiut, la 
mortalité infantile est surtout d'ordre endogène. Les modifications des conditions d'accouchement ainsi que la vraisemblable régression de l'infanticide féminin ont abaissé le taux de mortalité infantile de $180 \%$ à $120 \%$, entre 1900 et 1960 (RobertLamblin, 1981-1982).

d) Le taux de reproduction et de croissance. Pour qu'une population ait un taux de croissance positif, il faut que le taux de natalité soit supérieur au taux de mortalité, ce qui n'est pas toujours le cas dans les populations de chasseurs-cueilleurs. A titre d'exemple, chez les Kung, le taux brut de reproduction est de 2,3 enfants par femmes et le taux de reproduction net est de 1,07 filles par femme. Ces éléments de la reproduction humaine conjugués aux données de mortalité induisent généralement un taux de croissance proche du zéro (cf. fig. 4).

Le problème de la densité de population paraît essentiel. Ainsi, une population de chasseur comme celles des Hadza de l'Est africain qui pourrait sembler proche des Kung par le mode de vie en diffère fortement par la réponse apportée aux pressions exercées par l'environnement. On constate une pyramide des âges différentes de celles des Kung, induite par une fertilité différentielle significative: 4,7 enfants par femmes chez les Kung et 6,15 chez les Hadza. Le faible taux de croissance chez les chasseurs-cueilleurs pourrait aussi s'expliquer par des capacités physiologiques plus faibles par rapport à d'autres populations. Ainsi, chez les Hazda, l'intervalle génésique n'est guère plus élevé que celui des Kung, mais le sevrage est plus précoce $d^{\prime} u n$ an et peut expliquer la difference en matière de fécondité. De plus, la période de fertilité semble plus longue chez les Hadza que chez les Kung et paraît corrélée - à taille égale - à une charge pondérale plus importante chez les femmes Hadza (BlurtonJones, 1992). 
Intervalle génésique

Aménorrhée

Durée avant la conception

Intervalle entre conception et naissance

Total

10 mois

3 mois

9 mois

22 mois

Intervalle entre naissances vivantes

Intervalle génésique

Taux de mortalité fotale

22 mois

$12 \%$

$12 \%$

Taux de stérilité

27,3 mois

Début de la période de fertilité féminine

Age des premières règles

Stérilité de l'adolescence

Age du début de la période fertile

15 ans

1 an

16 ans

Durée de la période reproductive

Espérance de vie féminine à la naissance 29 ans

Age du début de la période fertile 16 ans

Durée de vie reproductive

13 ans

Nombre de naissances vivantes par femmes

Durée de vie reproductive

Intervalle entre naissances vivantes

Taux de mortalité maternelle

Nombre de naissances vivantes par femme

13 ans

27,3 mois

$10 \%$

4,7 enfants

Nombre d'enfants utiles par femme

Nombre de naissances vivantes par femme

Taux de mortalité infantile

4,7 enfants

$50 \%$

2,35 enfants

Nombre d'enfants utiles par femme

Taux de croissance annuel de la population

$0,73 \%$

Fig. 4. - Estimation de la croissance potentielle des populations de chasseurs-cueilleurs

(d'après Hassan, 1975).

B) Les facteurs culturels. - C'est davantage par l'intermédiaire de comportements sociaux que par l'action directe du milieu que va s'établir l'équilibre entre la taille de la population et les ressources dont elle dispose. Au rapport naissances/décès, va se rajou- 
ter l'émigration et l'immigration qui prennent des formes variables selon les sociétés. Ces migrations peuvent se faire sous forme volontaire comme le mariage et l'adoption, ou de force comme la capture de prisonniers qui sont généralement des femmes. Des exemples ont été fournis par Bunzel (1938) au sujet d'Indiens d'Amérique dụ Nord pour lesquels l'intégration de prisonnières à l'économie matrimoniale était un moyen important de pratique exogame.

De nombreuses règles sociales vont fournir des réponses aux contraintes écologiques. La première consiste à se débarrasser de ce qui remettrait en cause l'équilibre démographique du groupe. C'est pourquoi on assiste à des éliminations d'individus non actifs (les très jeunes et les très âgés). Comme l'explique Sahlins (1980), ces individus ne perturbent pas l'équilibre du groupe par la surcharge alimentaire que leur présence occasionne, mais parce qu'ils diminuent les capacités de mobilité de celui-ci.

a) L'infanticide. Bien que ce comportement soit fréquemment décrit par les anthropologues parmi les populations de chasseurs-cueilleurs, les statistiques sont toujours extrêmement difficiles à établir dans la mesure où les gouvernements et les missionnaires se sont attachés à les supprimer officiellement (pour prouver l'impact de la modernité et de l'évangélisation). C'est par l'étude des sex-ratio qu'il est possible de mettre l'infanticide différentiel en évidence.

En règle générale, les critères présidant à l'élimination des nouveau-nés s'avèrent variables d'une société de chasseurs-cueilleurs à l'autre. Par ordre décroissant, ce sont d'abord les nouveau-nés mal formés qui sont éliminés systématiquement, puis souvent, un des deux jumeaux, et parfois une partie des enfants de sexe féminin. Les explications ne sont pas univoques mais on constate par exemple, que dans bon nombre de sociétés, la naissance simultanée de 
2 enfants est vécue comme quelque chose d'anormal et de mauvaise augure en rappelant la dimension animale de l'homme: "Seuls les animaux portent plus d'un rejeton à la fois. »

Des différences de comportements s'observent entre sociétés. Si chez les Esquimaux Netsinik l'infanticide des filles est très élevé, par contre, chez les Kung comme d'ailleurs chez les Pygmées Mbuti, il est très faible (moins de $1 \%$ ) et semble être indépendant du sexe et ne s'appliquer qu'aux enfants mal formés. Chez les récolteurs de Sago en Nouvelle-Guinée, les filles dont la naissance survient trop rapidement après celle de l'enfant précédent sont éliminées. Dans cette population, le nombre moyen de naissances est de 5,3 enfants et la mortalité infantile y est très élevée avec un taux de $430 \%$ dont $110 \%$ sont attribués à l'infanticide. Deux fois plus de filles que de garçons sont éliminées. Dans cette population, $85 \%$ des apports caloriques proviennent du sagoutier. Or, contrairement à des populations comme les Aborigènes australiens et les Esquimaux, où les hommes en tant que chasseurs et pêcheurs sont les plus actifs, chez les cueilleurs de Sago, ce sont les femmes qui remplissent la fonction économique principale (une femme peut récolter en un jour de quoi nourrir 17 personnes). La disparition des enfants en bas âge par infanticide est élevée ce qui conduit à s'interroger sur les moyens de gérer l'adéquation optimale entre population et ressources. Il existe deux hypothèses concernant l'infanticide des filles. La première postule qu'il s'intègre dans une politique démographique à court terme visant à réduire la taille de la population en éliminant les individus les moins utiles; la seconde explique qu'il s'agit plutôt d'une stratégie sur le long terme dont l'objectif est de réguler le taux net de reproduction. Dans d'autres populations, on peut aussi observer des sex-ratio déséquilibrés. Ainsi, chez les Aborigènes australiens, Birdsell a trouvé un sex-ratio 
de $150 \%$ dans un ensemble de 1194 familles (Birdsell, 1973), montrant que $15 \%$ des filles ont été éliminées à la naissance. Dans ce cas précis, l'intensité de l'infanticide féminin est déterminé par la dimension de la fratrie puisque davantage de filles sont éliminées dans les familles nombreuses (le sex-ratio atteint $260 \%$ au sein des fratries de plus de 5 enfants).

D'autres facteurs, comme les structures sociales jouent aussi un rôle. Dans le cas des Indiens d'Amérique du Sud, on observe des différences interethniques. La comparaison des comportements en matière d'infanticide entre les Yanomami et les Jivaros est donnée dans le tableau suivant:

Yanomami

Jivaros

Enfants mal formés

$2^{e}$ jumeau

Filles

Légende : - infanticide absent ; + infanticide occasionnel ; ++ infanticide systématique.

Pour ces deux ethnies, ce sont les pratiques guerrières associées au type de résidence qui vont influencer le type d'infanticide. Ainsi, chez les Jivaros, il n'y a pas d'infanticide féminin parce que la résidence est matrilocale, les filles procurant des gendres (le sexratio est de $85 \%$ chez les adolescents). Par contre chez les Yanomami où la résidence est patrilocale le sex-ratio est de $142 \%$.

D'autres exemples concernant le poids du culturel sur l'infanticide peuvent être cités, comme celui des Esquimaux Caribou chez lesquels davantage de nouveau-nés masculins seraient éliminés en raison du nombre peu important de chasseurs nécessaires pour 
l'équilibre social du groupe. Ceci apparaît dans la transformation du sex-ratio à la naissance de $165 \%$ en un sex-ratio de $80 \%$ chez les adolescents. Inversement, dans d'autres sociétés Inuits, lorsqu'il y a surnombre de filles et un besoin plus élevé de chasseurs, certaines réponses culturelles autres que l'infanticide ont été trouvées. Chez les Esquimaux Ammassalimiut, on a observé des "changement de sexe", c'est-à-dire que certaines filles sont élevées comme des garçons et remplissent une partie de la fonction sociale de ceux-ci en devenant chasseurs (RobertLamblin, 1978).

b) La mortalité sociale. La mortalité sociale, outre l'infanticide, revêt généralement les formes suivantes :

- le géronticide: les personnes âgées sont tuées ou se suicident (Esquimaux).

- l'invalidicide : élimination des sujets invalides;

- la guerre : elle accroît le taux de mortalité chez les jeunes hommes, mais peut être en partie compensée par la prise de captifs ;

- le rituel : sacrifice et cannibalisme ;

- les décès attribués à la magie et à la possession : la mort est due à une perte de nature psychologique de la volonté de vivre.

Cette mortalité sociale contribue surtout à la régulation démographique de presque toutes les populations de chasseurs-cueilleurs. Néanmoins, des différences importantes sont observables d'une population à l'autre. Dans un groupe peu belliqueux, comme les Kung, les morts violentes ne représentent que 5 à $10 \%$ des décès (meurtres, accidents, infanticides) alors que les maladies infectieuses et parasitaires représentent $80 \%$ et les maladies de dégénérescence $10 \%$. Dans une population très guerrière comme celle des Yanomami, la cause principale de décès des hommes est la guerre (cf. fig. 5) et les décès pour causes «magiques » sont aussi importants que ceux dus aux 
maladies épidémiques (Chagnon, 1974). Il convient de souligner que les décès dus à la guerre sont vécus comme naturels alors que les autres formes de décès, et principalement les épidémies, apparaissent comme résultant d'une violation de tabous.

Les phénomènes d'acculturation vont modifier la répartition des causes de décès. On a pu étudier cette répartition pour deux groupes de Jivaros (les Shuar et les Aguaruna) en fonction de leur isolement par rapport à d'autres sociétés. Dans le groupe très isolé, $36 \%$ des décès des hommes adultes sont dus à des morts violentes, alors que chez les autres, ce type de décès ne représente que $16 \%$. En revanche, dans le second groupe, la sorcellerie cause $53 \%$ des décès masculins.

\begin{tabular}{|c|c|c|c|c|}
\hline \multirow[b]{2}{*}{$\begin{array}{l}\text { Informations } \\
\text { fournies }\end{array}$} & \multicolumn{2}{|c|}{$\begin{array}{l}\text { Village } \\
\text { Shamatari }\end{array}$} & \multicolumn{2}{|c|}{$\begin{array}{c}\text { Village } \\
\text { Namovei-teri }\end{array}$} \\
\hline & $\begin{array}{l}\text { Hom- } \\
\text { mes }\end{array}$ & $\begin{array}{l}\text { Fem- } \\
\text { mes }\end{array}$ & $\begin{array}{l}\text { Hom- } \\
\text { mes }\end{array}$ & $\begin{array}{l}\text { Fem- } \\
\text { mes }\end{array}$ \\
\hline Causes « naturelles » & 0 & 0 & 0 & 2 \\
\hline Épidémies & 32 & 78 & 72 & 81 \\
\hline Dysenterie & 1 & 1 & 14 & 5 \\
\hline Guerre & 52 & 5 & 44 & 9 \\
\hline Duels & 1 & 1 & 5 & 0 \\
\hline Tuée par le mari & 0 & 0 & 0 & 1 \\
\hline Morsure de serpent & 1 & 3 & 4 & 2 \\
\hline Accouchement & 0 & 0 & 0 & 1 \\
\hline Infection respiratoire & 1 & 5 & 3 & 0 \\
\hline Hayaheri (douleur & & & & \\
\hline abdominale) & 0 & 1 & 6 & 1 \\
\hline Vieillesse & 7 & 4 & 8 & 3 \\
\hline Sorcellerie & 27 & 11 & 15 & 5 \\
\hline Chute d'arbre & 1 & 0 & 1 & 0 \\
\hline Hekura (possession) & 6 & 5 & 13 & 20 \\
\hline Rougeole & 0 & 1 & 0 & 0 \\
\hline
\end{tabular}

Fig. 5. - Causes des décès chez les Yanomami selon leur niveau d'acculturation

(d'après Chagnon, 1974). 
La guerre, en plus des réductions démographiques qu'elle opère, s'associe à un certain nombre de pratiques sociales assurant la cohésion du groupe. Nous pouvons citer l'endo-cannibalisme des Yanomami qui consiste à enterrer à l'intérieur de soi-même un membre de sa famille mort au combat. Les cendres du mort sont mélangés avec des fruits et mangés. Les Yanomami sont des guerriers pratiquant les rapts de femmes dans les tribus ennemies, ils associent la mort des veuves des guerriers à l'endo-cannibalisme, afin que celles-ci ne soient pas capturées par l'ennemi. Du fait de leur veuvage, ces femmes deviennent moins protégées et leur capture pourraient en faire des mères dont les enfants deviendraient des ennemis (résidence patrilocale). Les papous de NouvelleGuinée pratiquent aussi l'endo-cannibalisme, mais d'une manière différente en consommant la cervelle du mort après l'avoir fait bouillir. La fonction symbolique demeure la même. L'endo-cannibalisme des papous provoque une maladie, le Kuru. De toute façon, le cannibalisme, quel que soit sa forme, n'a jamais de finalité alimentaire. Signalons à ce sujet une simulation «écologique " de Dornstreich (1974) montrant la valeur nutritionnelle du cannibalisme qui, selon cet auteur serait théoriquement une bonne stratégie alimentaire dans les zones moyennement peuplées de Nouvelle-Guinée. Pour un groupe de100 personnes, la consommation de 10 adultes par an compenserait l'apport protéique obtenu par un élevage de porc. En revanche, la pratique de la chasse «aux têtes » que l'on rencontre chez les Marind de Nouvelle-Guinée a une répercussion plus importante sur le plan démographique. Van Der Kroef (1952) a calcule que, pour répondre aux normes sociales, 15000 guerriers doivent tuer 15000 ennemis dans les autres tribus. On se doute que cette pratique guerrière engendre autant d'actions de vengeance qui ne feront qu'amplifier les destructions de populations. 
Un modèle pourrait exprimer la dynamique bioculturelle de la régulation démographique construit à partir de données recueillies sur les Eipo de NouvelleGuinée. Bien que ces derniers ne soient pas chasseurscueilleurs (ils sont horticulteurs et éleveurs de porc), le modèle s'applique bien aux populations de chasseurs-cueilleurs (cf. fig. 6).

c) L'impact du changement. C'est généralement au contact d'autres sociétés que s'effectue une accélération de la transformation du mode de vie. Ce contact peut être progressif ou brutal. Dans le cas d'un contact avec des peuples colonisateurs, la transformation s'est souvent soldée par une destruction de la population concernée. Citons les Indiens d'Amérique du Nord et du Sud, les Aborigènes d'Australie (fortement massacrés en 1870) ou ceux de Tasmanie (le dernier est décédé en 1878).

La destruction s'effectue souvent de manière indirecte. En Australie, l'économie de subsistance des Aborigènes reposait sur la consommation de graminées sauvages et de petits marsupiaux $(70 \%$ de l'alimentation). L'introduction du mouton et du lapin, conjuguée à la destruction systématique des kangourous et des émeus par les blancs, ont déstructuré l'écosystème. La conjonction de ces deux phénomènes (extermination de la population et déstructuration de l'écosystème) ont réduit la population aborigène de 300000 en 1878 à 60000 en 1930 (Fraser, 1986). Très peu de groupes ont pu s'adapter aux brutales modifications sociales et écologiques. Citons les Guarakis du Paraguay devenus voleurs de bétail ou les Esquimaux qui se sont intégrés dans des circuits commerciaux en pratiquant la vente de produits très recherchés (fourrures). Il s'agit d'un cas où le contact avec d'autres populations a permis de renforcer l'activité de chasse. La raison est d'ordre purement économique, car un chasseur gagne le double d'un employé pour un temps de travail moindre. 
Puberté tardive (premières règles vers 17 ans)

Première conception tardive (généralement après 20 ans) $=$ Raccourcissement de la période reproductive

Fréquence des relations sexuelles peu élevée (estimée à une fois par semaine)

Faible chance de conception

Allaitement durant plus de trois ans (les règles reviennent après quinze mois)

Post-partum coitus taboo (durant deux trois ans)

Faible chance de conception

Infanticide

Faible nombre d'enfants

Infanticide féminin préférentiel

\section{Diminution de la population féminine}

Décès naturels (maladie, accident, vieillesse)

Diminution de la population

(mortalité masculine plus élevée

Décès au combat que la mortalité féminine)

Diminution de la population

(mortalité masculine plus élevée

Suicide que la mortalité féminine)

Diminution de la population

(mortalité féminine plus élevée Meurtre des « sorcières » que la mortalité masculine)

\section{Diminution de la population}

(mortalité féminine plus élevée que la mortalité masculine)

Fig. 6. -- Modèle de la régulation démographique dans les populations de chasseurs-cueilleurs (d'après Mc Elroy et Townsend, 1989). 
En général, l'aspect le plus important du changement chez les chasseurs-cueilleurs est le passage du nomadisme à la sédentarisation. Les conséquences de ce changement ont pu prendre des formes de déstructuration extrêmes comme chez les Iks d'Ouganda. Ces chasseurs-cueilleurs ont été chassés de leur région traditionnelle de chasse par le gouvernement. Cette rupture avec leur mode de vie traditionnel a provoqué une déstructuration sociale très importante. La survie est devenue une affaire uniquement individuelle et toutes les valeurs du groupe ont été abandonnées, ce qui s'est manifesté, entre autre, par un refus de nourrir les vieillards et les enfants. L'impact démographique du passage du nomadisme à la sédentarisation peut d'ailleurs être modélisé (fig. 7).

La régulation démographique des populations de chasseurs-cueilleurs s'effectue par une fécondité

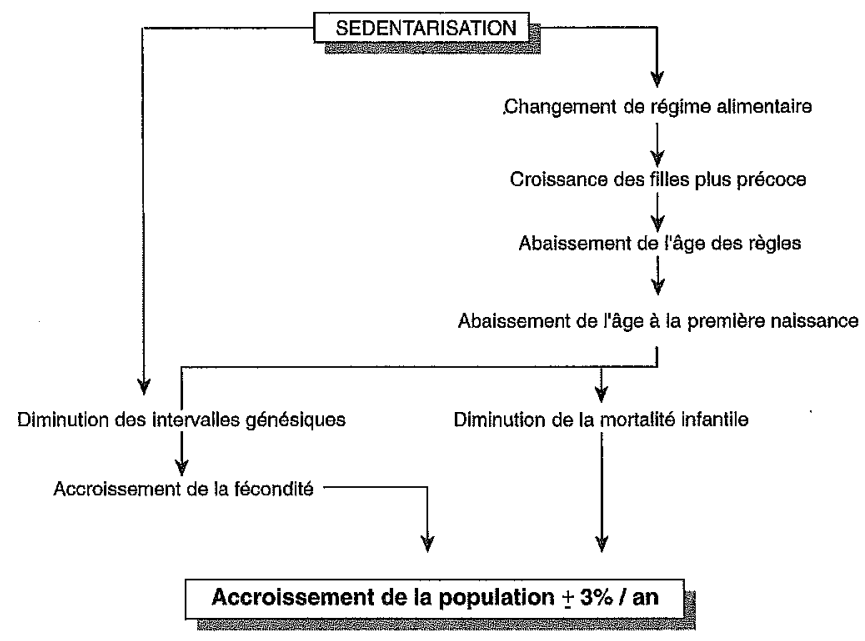

Fig. 7. - Modèle de l'accroissement démographique après la phase de sédentarisation. 


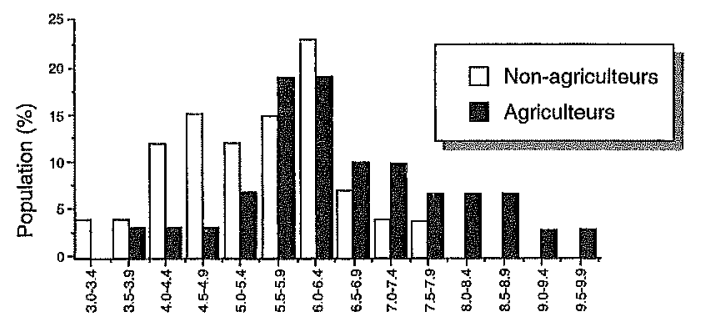

Taux de fertilité

Fig. 8. - Comparaison du pourcentage de distribution de la descendance finale entre différentes populations d'agriculteurs et de non-agriculteurs (chasseurs-cueilleurs) (d'après Bentley, 1993).

moyennement élevée, comprise entre celles des populations des pays industrialisés ( 2 à 3 enfants) et des populations de cultivateurs du Tiers Monde $(6$ à 8 enfants), conjuguée à une forte mortalité. Le contact de plus en plus fréquent de ces populations avec d'autres entraîne un phénomène général de déstructuration de ces groupes. La sédentarisation entraîne de moins bonnes réponses adaptatives du groupe aux maladies et une diminution de l'infanticide sélectif, ce qui minimise les pressions de l'environnement sur le groupe. Les populations de chasseurs-cueilleurs qui n'ont pas été détruites (cf. supra), dont le taux de croissance démographique était proche de zéro lorsqu'elles pratiquaient le nomadisme, vont augmenter sous l'effet de la sédentarisation (fig. 8). Leur rythme de croissance atteindra alors celui des populations d'agriculteurs.

\section{II. - Les populations rurales des pays en développement : l'exemple africain}

Le comité sur la population du National Research Council a réalisé une série d'études synthétiques (sous 
les auspices d'un groupe d'experts composé de démographes, anthropologues et biologistes) destinée à faire le point sur la dynamique des populations africaines subsahariennes, à partir des données issues des enquêtes EDS (enquêtes démographie et santé). Six rapports ont été publiés qui analysent respectivement la fécondité des adolescentes, les pratiques en matière de contraception, la santé et la mortalité des jeunes enfants, l'économie et ses effets démographiques et enfin deux monographies, l'une sur le Kenya et l'autre sur le Sénégal. En effet, en Afrique, les démographes ont eu des difficultés pour obtenir des données de qualité avant les enquêtes EDs. Malgré tout, celles-ci ne sous-estiment pas les nombreuses difficultés qui persistent, telles que l'imprécision des dates de naissances ou les erreurs dans l'échelonnement des naissances. Les recherches mettent partout en évidence une diminution de la mortalité générale et infantile, une amorce de la baisse de la fécondité seulement dans certains pays ou régions et quelques évolutions dans les comportements matrimoniaux. Ces résultats ne permettent cependant pas de dégager de tendances dont les conséquences semblent encore difficiles à cerner avec précision.

Nous sommes au début du deuxième stade de la transition démographique (amorce du déclin de la fécondité) et l'analyse des modalités d'évolution du mariage et de la fécondité, qui requiert le concours de spécialistes de différentes disciplines, s'inscrit assez bien dans une approche d'anthropologie démographique.

1. Les déterminants de la nuptialité. - En Afrique, il est difficile d'envisager l'âge au mariage comme une variable dont la mesure peut s'interpréter facilement. Il y a plusieurs stades dans le chemin que parcourent deux individus avant de se marier et qui s'échelonne généralement sur plusieurs années. Cette 
complexité est bien entendu un handicap pour obtenir des données précises et facilement interprétables. La compréhension de l'évolution d'un tel phénomène implique alors une approche d'anthropologie démographique, à partir de petites populations, et se situe au croisement de méthodes quantitatives et qualitatives.

Une étude réalisée par Gilles Pison (1997) sur les Peul Bandé de Bandiagarra (zone de Bandafassi, sudest du Sénégal, près de la frontière guinéenne) exploite des données recueillies sur 25 villages, d'un effectif total de 4100 personnes en 1985. La répartition par classe d'âge et par sexe montre qu'au-delà de 20 ans les femmes sont plus nombreuses que les hommes. L'écart est de $20 \%$ en moyenne. Dans cette région de Bandafassi, comme d'ailleurs un peu partout en Afrique, il existe de fortes variations des comportements matrimoniaux et des naissances prénuptiales. Si l'on examine l'âge moyen au mariage et celui à la première naissance on constate de fortes différences entre les ethnies qui résident dans cette zone. Les femmes Peuls se marient à 16,6 ans et elles ont leur première naissance à 17,9 ans alors que le phénomène est plus tardif chez les Malinkés avec respectivement de 19,4 et 20,6. En revanche, chez les Bediks, les âges au mariage et à la première naissance sont de 19 et 19,2. Ces chiffres mettent bien en évidence l'importance des naissances hors mariage pour le premier enfant et montrent que la question de l'âge au mariage comme déterminant de la fécondité n'est pas toujours un paramètre fiable dans les analyses. Dans cette région, la polygamie est importante puisqu'il y a 1,8 femme mariée pour un homme marié. L'écart d'âge au premier mariage est de dix ans, les femmes se mariant en moyenne à 15 ans et les hommes à 25 ans. Le remariage des hommes s'effectuant entre 25 et 45 ans. D'une façon générale les ruptures d'unions sont nombreuses (26\% des femmes divor- 
cent dans les trois premières années de leur union), mais elles sont quasiment toujours suivies d'un remariage. Le célibat définitif est donc faible dans cette population et le délai de remariage est généralement court, que ce soit après veuvage ou divorce. Le lévirat est également très courant chez ces agriculteurs PeulsBandé. Pour ces populations islamisées, le temps d'attente pour un remariage est codifié à quatre mois et dix jours. Une caractéristique de cette population est le mariage apparenté, en particulier celui de l'homme avec sa cousine croisée matrilatérale (fille du frère de la mère).

Si la relative stabilité de la polygamie s'explique par la perpétuation de comportements traditionnels, elle est également très liée à un système démographique. La polygamie correspond au fait que certains hommes mariés ont plusieurs femmes et que toutes les femmes n'ont qu'un seul mari. Il y a pourtant égalité numérique entre les sexes qui ne sont pas affectés différemment par des guerres ou des migrations. Il n'y a donc pas d'excédent de femmes dans la population, le choix des femmes ne se fait pas particulièrement dans les populations voisines et le célibat masculin est très faible. En fait, la seule explication plausible est mécanique. C'est parce qu'il y a écart d'âge au mariage important entre les deux sexes qu'il peut y avoir polygamie. Le deuxième élément favorisant est le fort pourcentage de remariage des femmes après veuvage. Ce processus fonctionne d'autant mieux que la population est jeune et qu'il y a un grand potentiel de femmes mariables, et qu'il existe parfois une émigration masculine doublée d'une surmortalité masculine (Pison, 1985).

Il est intéressant de remarquer que ces résultats ne diffèrent pas vraiment de ceux d'une enquête en cours au sud du Cameroun, réalisée sur l'ensemble de treize villages appartenant à la zone géographique de la boucle du fleuve Ntem, près de la frontière de la 
Guinée équatoriale. La zone d'étude relève administrativement de l'arrondissement de $\mathrm{Ma}$ 'an (petit cheflieu de 658 habitants) situé dans le département du Ntem. Il s'agit d'une région faiblement peuplée (environ $2 \mathrm{hab} . / \mathrm{km}^{2}$, RGP 1987) où les populations pratiquent l'essartage comme agrosystème forestier de zone tropicale humide. Cette zone de transition allie la forêt, dégradée le long des axes routiers par l'occupation humaine persistante, et la culture cacaoyère. La population de la région s'étire donc le long de l'unique piste reliant tous les villages au cheflieu d'arrondissement (Ma'an). Elle se compose de deux ethnies, les Ntumu et les Mvae appartenant au même groupe linguistique. L'intercompréhension linguistique, l'utilisation des mêmes référents rituels et symboliques ainsi que les mariages mixtes et la proximité résidentielle permettent de ne pas faire de distinction entre ces deux ethnies. Les villages sont généralement monoclaniques et constitués de plusieurs hameaux. Les populations pratiquent le mariage de type exogamique à résidence virilocale.

Les villages ont des effectifs très inégaux, allant de 23 personnes pour Akom (le plus petit) à 271 personnes pour Mvilimengale (le plus grand). La population comprend 1674 personnes dont 847 de sexe masculin $(50,6 \%)$ et 827 de sexe féminin $(49,4 \%)$, réparties en 225 ménages. Il s'agit d'une population jeune puisque $45,9 \%$ des personnes ont moins de 15 ans, ce qui correspond aux normes trouvées habituellement dans les pays en développement ayant une fécondité élevée.

La répartition de la population par classes d'âge et sexe (pyramide des populations) fait apparaitre un déficit populationnel après 25 ans, plus prononcé pour les hommes entre 35 et 50 ans, que l'on peut attribuer à l'effet des migrations.

Parmi la population de plus de 15 ans, on constate qu'il y a $54 \%$ d'hommes célibataires pour seule- 
ment $41,5 \%$ chez les femmes. Ce résultat traduit les comportements polygames d'une fraction de la population, qui est a été estimée à $16 \%$. Quelques éléments permettent de caractériser les unions. L'âge moyen des hommes au premier mariage est de 25,1 ans et celui des femmes de 19,1 ans lorsqu'elles se marient avec un conjoint célibataire. En revanche, le remariage des hommes se situe à une moyenne de 39 ans. Si l'on examine l'écart d'âge moyen au premier mariage, il est de six ans. Mis à part la plus faible proportion de mariages polygynes et l'âge au mariage des femmes plus tardif (d'ailleurs peu pertinent vu que la quasi-totalité des femmes ont des naissances prénuptiales), les différences sont peu sensibles.

Même s'il est difficile de comparer deux populations qui vivent dans des conditions écologiques dissemblables, on notera toutefois que les comportements généraux en matière de nuptialité sont assez proches et que la question de l'entrée dans la vie matrimoniale et féconde ne semble pas présenter d'évolution notable qui pourrait influencer la fécondité.

Les données sur ces deux populations appellent quelques remarques plus générales concernant le mariage africain, que nous avons empruntées à Van de Walle (1996). Pour les démographes la nuptialité est intéressante uniquement parce qu'elle peut avoir une incidence sur la fécondité. Dans les populations de fécondité naturelle (ce qui est encore largement le cas en Afrique), ils considèrent que l'âge au premier mariage va influer sur la durée d'exposition au risque de grossesse et en fin de compte sur la descendance finale. Cela est vrai à condition qu'il n'y ait pas, ou très peu, de naissances illégitimes avant le mariage. $\mathrm{Ce}$ qui n'est pas ou plus le cas en Afrique aujourd'hui. Il faut en effet noter l'importance des activités sexuelles prénuptiales. Dire qu'il existe un 
recul de l'âge au mariage n'est pas suffisant car il est souvent compensé par des unions libres, souvent fécondes. A propos du mariage, il faut aussi savoir qu'il existe une grande diversité de types d'union: coutumier, religieux, civil... Il faut également tenir compte des périodes de concubinage, souvent nombreuses et diverses, ainsi que des périodes de veuvage, des remariages, de la pratique du lévirat et enfin de la polygynie.

Ces différents éléments montrent que le mariage doit être abordé de façon quantitative (par les démographes) et de façon qualitative (par l'anthropologue). De plus l'extrême diversité des comportements rend les comparaisons difficiles. A cela se rajoute comme partout sur le continent, la question de la fiabilité des dates, surtout s'il s'agit d'enquêtes rétrospectives. Il est donc nécessaire de pouvoir effectuer des études anthropologiques fines pour pouvoir apprécier la réalité de ce phénomène. L'examen des résultats des Enquêtes DémographieSanté (EDS) montre que l'impact de l'âge au premier mariage sur la fécondité semble faible, voire même, inexistant. Par contre, à terme on peut très bien imaginer que la sexualité prénuptiale pourrait aussi servir de période d'essai pour la contraception. En effet, les jeunes femmes peuvent avoir intérêt à retarder leur première naissance pour continuer leurs études. Ces différents aspects sont complexes et méritent une attention soutenue car c'est leur analyse qui permettra d'apprécier la possible évolution des populations africaines mais cela nécessitera de relier données statistiques et résultats anthropologiques.

2. La gestion de la fécondité. - La discussion sur l'importance de la notion d'âge pour l'explication de la polygamie renvoie à un autre exemple qui montre en quoi les fondements culturels d'une population 
peuvent avoir des traductions sur sa structure démographique.

Les systèmes d'âges semblent les plus répandus et c'est seulement en Afrique de l'Est que l'on rencontre des systèmes générationnels. L'exemple des MeruTigania-Igembe du Kenya (Peatrick, 1994) est intéressant en ce sens qu'il met bien en évidence la difficulté de fonctionnement d'un groupe qui aujourd'hui serait organisé selon un système générationnel. L'auteur décrit avec précision les différents degrés que tout individu doit franchir entre sa naissance et sa mort et les conséquences et limites de fonctionnement d'un tel système.

L'une des conséquences est celle montrant l'impact de ce système sur la démographie du groupe. En effet, chez les Meru, les processus biologiques sont dépendants de l'organisation sociale du groupe et une femme ne peut procréer qu'après avoir subi son initiation pour entrer dans la catégorie reconnue. Toute grossesse antérieure à cette initiation ne pouvant être acceptée aux yeux de la communauté, il y avait nécessairement avortement ou même infanticide. De la même façon, l'initiation tardive des filles qui doivent entrer dans la catégorie des initiants puis des futures épouses, suivie souvent d'une période de réclusion, favorise le mariage tardif des femmes et donc diminue d'autant la période de fécondabilité de la femme socialement reconnue. Dans le même esprit, dès que l'aîné d'une famille avait lui-même des enfants, cela obligeait sa mère à mettre un terme à sa descendance, ce qui est encore une raison supplémentaire pouvant influer sur la descendance finale des familles. Ce système présente des limites qui sont en liaison avec des approches démographiques. Par exemple, la correspondance entre l'âge réel et la catégorie d'appartenance était parfois difficile à réaliser et pouvait même aller jusqu'à provoquer la remise en cause des classes d'âges. 
Avec six enfants par femme en moyenne, la fécondité en Afrique subsaharienne reste la plus élevée du monde. C'est la seule région du monde qui n'a pas encore connu de baisse générale de la fécondité, fortement conditionnée par la culture et les situations de développement économique. Dans ces pays, l'âge précoce du mariage et son intensité favorisent une forte fécondité légitime, d'ailleurs souvent compensé par une mortalité élevée. Ce qui est intéressant, au-delà de la simple mesure du recul de l'âge au mariage comme premier élément de la phase 2 de la transition, c'est d'y associer des explications qui relèvent d'interactions complexes entre les comportements individuels et l'environnement socioéconomique. Pour reprendre les exemples cités dans la partie précédente, on relèvera un indice synthétique de fécondité (ISF) de 6,2 pour la période 1981-1991 dans la population de Bandafassi et de 5,7 en 1997 dans la population du Ntem.

Lorsque la femme se marie jeune, qu'elle a un enfant rapidement et qu'elle poursuit sa vie génésique sans interruption jusqu'à la ménopause, on comprend bien pourquoi la fécondité est élevée. En l'absence de contraception, seules les périodes d'aménorrhée et l'importante durée d'allaitement allongent les intervalles entre naissances et réduisent un peu la dimension de la descendance possible des femmes. L'usage de la contraception est encore très faible, et il a pour objectif d'allonger la durée entre les grossesses. A l'inverse, la diminution de la durée de l'allaitement se répand plus rapidement et son action sur l'espacement des naissances, loin d'être compensé par l'usage de contraceptifs, provoque plutôt une augmentation de la dimension de la famille. Par exemple, dans le Ntem, si les préservatifs sont connus de la population, une enquête préliminaire montre qu'ils sont très peu utilisés et souvent pour des motifs qui ne concernent pas la limitation des naissances. Les 
quelques utilisateurs sont plutôt les hommes qui, pendant la période d'allaitement de leur femme, préfèrent l'utilisation de ce moyen à l'abstinence. Un autre motif concerne l'usage que peuvent en faire les hommes dans la fréquentation des prostituées ou d'autres partenaires, car il existe dans cette population une forte mobilité sexuelle.

Il y a en fait une multiplicité de facteurs qui tous concourent à maintenir un niveau élevé de fécondité. Par exemple, Paul Pascon (1986) défend le point de vue qu'il existe, dans une société rurale et/ou traditionnelle, à un moment donné, une liaison très étroite entre les comportements de procréation et l'organisation de la production. Il émet l'hypothèse que dans des sociétés soumises au poids des facteurs d'environnement naturels et qui n'ont pas atteint un fort niveau de développement technologique, une fécondité élevée et un mode d'organisation familiale basé sur le nombre et la solidarité est la seule réponse possible pour que ces populations puissent vivre au mieux. Autrement dit, pour produire plus, il faut avoir plus de bras à sa disposition. Et même si le nombre de personnes à nourrir est plus important, le groupe à l'arrivée est gagnant, car une forte fécondité doit l'aider à asseoir sa puissance.

Le cas du temps d'utilisation de l'eau destiné à l'irrigation est exemplaire. Dans une plaine comme le Haouz, aux environs de la ville de Marrakech (sud marocain), le climat et la qualité des sols empêchent toute agriculture productive en l'absence d'irrigation. Seules les terres irriguées peuvent permettre une production agricole, mais les possibilités d'accès à l'eau sont réduites et il existe souvent un système d'irrigation par des moyens traditionnels. Ce sont des canaux qui desservent l'ensemble des terres mises en cultures des différents propriétaires et exploitants. Ce qui est important, c'est le temps d'utilisation de l'eau réparti entre chaque agriculteur, en fonction de 
la dimension de sa famille ou de la taille de son groupe. Donc, avoir beaucoup d'enfants, c'est obtenir un temps plus important pour irriguer ses terres, avoir ainsi la possibilité de travailler de plus grandes surfaces agricoles et en dernier ressort avoir un volume de production plus important. D'ailleurs, tous les exemples vont dans ce sens, comme l'attribution de terres en fonction de la taille du groupe.

Cette relation entre la dimension de la famille n'est d'ailleurs pas une spécificité des pays en développement, puisque dans les populations rurales occidentales des siècles précédents, il existait une différence dans la taille de la famille (ou du groupe domestique) des agriculteurs selon qu'ils étaient métayers, fermiers ou propriétaires. Les métayers avaient intérêt à avoir des familles nombreuses dans le but d'accroître la production et donc leurs revenus, à l'opposé des propriétaires dont l'intérêt est de limiter leur descendance s'ils ne veulent pas trop morceler leur patrimoine.

Un autre exemple pris au Maroc montre cependant la primauté des facteurs culturels sur le niveau économique. Dans une étude sur Marrakech, les attitudes et comportements en matière de soins apportés au jeune enfant ont été examinés selon les groupes professionnels. Quatre groupes ont été choisis : tertiaire, artisans/commerçants, ouvriers, divers. Une définition limitative des professions du tertiaire a été retenue, ne comprenant que les carrières des services publics, de l'enseignement et des banques. Dans un pays où la situation de l'emploi est aléatoire, elles se démarquent de l'ensemble des autres professions par leur caractère permanent et représentent un groupe social en développement rapide. Par contre, les professions de l'artisanat et du commerce, habituellement classées dans le secteur tertiaire, ont été considérées comme un groupe particulier. En effet, dans un centre urbain comme Marrakech, elles correspondent à une activité 
traditionnelle exercée par un nombre important d'individus formant un ensemble social nettement défini et cependant très différent des autres catégories du tertiaire. Si la définition classique des groupes professionnels avait été respecté, le comportement démographique moderne des femmes du secteur tertiaire, par l'absence de surmortalité exogène, n'aurait pas été mis en évidence. On notera que les groupes tertiaires et artisans-commerçants, bien que les plus proches et les plus favorisés sur le plan économique, diffèrent considérablement dans leurs comportements. Les femmes dont le conjoint exerce une profession du tertiaire accouchent plus fréquemment à l'hôpital et allaitent leurs enfants moins longtemps que les femmes du groupe artisan-commerçant. Ce résultat illustre parfaitement l'influence des mentalités et des habitudes culturelles qu'il serait dommageable de négliger dans ce type de travaux.

3. La mortalité infantile et le rôle du milieu. - La dynamique bioculturelle ne peut s'appréhender en dehors du contexte particulier constitué par le milieu dans lequel les sociétés vivent et se reproduisent. D'une part, le milieu contraint l'homme à des adaptations biologiques, mais d'autre part, l'homme modifie son environnement qui, de contrainte, devient lieu d'intégration. Cela est d'autant plus net chez les populations vivant dans des milieux naturels plutôt hostiles pour l'homme et qui n'ont souvent pas d'importants moyens technologiques à leur disposition. Ce sont évidemment des situations que l'on retrouve dans les pays en développement.

Examinons l'impact des conditions de milieu et des comportements des populations sur la démographie et l'état de santé des populations, dans la zone d'El Azzouzia, située en limite de la ville de Marrakech (Maroc) qui est la zone d'épandage des eaux usées de la ville et de sa zone industrielle. Dans une enquête sur 
la mortalité infantile effectuée à partir de questionnaires auprès des femmes qui venaient en consultation dans les différents dispensaires de la ville, les résultats ont montré qu'au-delà d'un chiffre moyen du taux global de mortalité infantile $(77 \%$ ), il y avait des différences notables selon les quartiers de la ville. En particulier, les chiffres les plus élevés (122\%) concernaient le dispensaire de Semlalia, situé dans un quartier qui pourtant ne présentait pas de conditions particulièrement défavorables. Une étude plus approfondie permit de s'apercevoir qu'une fraction des femmes qui consultaient à ce dispensaire venaient de la zone contiguë d'El Azzouzia, située à l'extérieur des limites de la municipalité. Une étude plus détaillée, effectuée sur cette zone (Hilali, 1986) permit d'expliquer cette situation singulière. La zone d'épandage d'El Azzouzia (sur $19 \mathrm{~km}^{2}$ ) offrait, dans une région aride et aux sols de mauvaise qualité, deux avantages pour y faire de l'agriculture : celle d'avoir d'une part de l'eau en quantité suffisante pour irriguer les terres $(3000$ ha de terres irriguées), et d'autre part une eau riche en matières organiques (et donc tenant lieu d'engrais) pour obtenir de meilleurs rendements de productions. Cette zone inhabitée vît, en peu de temps, l'installation de paysans immigrés venus de zones rurales plus défavorisées. Ils fondèrent ainsi plusieurs douars (d'un effectif total de 2200 personnes) et pratiquèrent une culture maraîchère qui pouvait facilement trouver acquéreur sur les marchés auprès de la clientèle citadine. Le revers de la médaille de cette relative aisance économique était que ces populations vivaient dans de mauvaises conditions sanitaires, car la zone n'était pas destinée à accueillir des habitations et donc pas viabilisée. Les conditions d'insalubrité (absence d'eau potable et d'électricité, proximité des puits et des latrines, proximité des hommes et du bétail) additionnées aux comportements encore très traditionnels d'une population restée très rurale dans ses activités autant que dans ses 
mentalités expliquent le risque important d'exposition des jeunes enfants (ceux les plus exposés aux agressions du milieu environnant) et en conséquence le fort taux de mortalité infantile relevé dans cette population.

Un autre exemple (Boukir, 1995) illustre les relations entre la campagne et la ville. A partir d'un questionnaire d'enquête soumis à 263 ménages de la Médina de Fès, et d'un questionnaire complémentaire, destiné à apprécier les motifs de la migration des ruraux en ville, le chercheur fait un état des lieux de la structure démographique, culturelle et socioéconomique de la population de la ville de Fès. Il analyse avec précision les mécanismes qui ont présidé au phénomène de «densification de la Médina » et fait ainsi apparaître toute l'ampleur des conséquences sur les conditions de vie des résidents. Puis il traite de la question de l'eau dans la ville et fait ressortir le déficit en eau potable, les difficultés d'extension du réseau et les problèmes posés par l'évacuation des eaux usées et leurs conséquences sur le milieu. La croissance démographique accompagnée d'investissements limités pour une population mal informée, a entraîné des dégradations des conditions d'assainissement et d'alimentation en eau et par conséquent de l'environnement sanitaire et hygiénique. Il évalue les conséquences d'un environnement dégradé sur la santé des populations, en particulier sur la mortalité des enfants. La mortalité par diarrhée est mesurée et l'approche de la perception de cette affection par les femmes est analysée, ce qui montre une fois de plus le poids des facteurs culturels.

\section{III. - Les populations rurales occidentales : l'exemple de Châteauponsac}

Dans le monde rural européen, les études d'anthropologie s'appuyent sur quelques principes essentiels, en particulier sur le fait que l'organisation sociale 
s'ordonne autour des processus de reproduction et de dévolution des biens (Augustins, 1989). En effet, les principes de filiation et d'alliance qui sont appliqués dans les sociétés exotiques sont différents dans les sociétés rurales occidentales. Ce n'est plus la parenté qui conditionne la transmission patrimoniale, mais plutôt l'inverse: l'héritage et le patrimoine qui lui sont associés permettent d'expliquer le mode de perpétuation du lignage dans les sociétés paysannes européennes en favorisant les alliances au sein des réseaux de parenté.

L'une des caractéristiques des campagnes européennes est le phénomène du dépeuplement qui s'est généralisé au lendemain de la première guerre mondiale. C'est la conséquence de l'exode rural et de la baisse régulière de la natalité dans les campagnes qui est apparue dès le XIX ${ }^{\mathrm{e}}$ siècle. Cette baisse de la fécondité est associée à deux facteurs principaux : l'âge au premier mariage et le taux de célibataires, auquel se rajoutait, en cas de "crise» démographique, un niveau de mortalité élevé qui libérait souvent des terres. Cette disponibilité de terres favorisait à son tour des unions plus nombreuses qui allaient augmenter la natalité générale et permettre de revenir à un état d'équilibre. Si l'ancien régime s'est caractérisé à la fois par un nombre restreint de mariages et par un nombre important de naissances illégitimes, le début $\mathrm{du} \mathrm{XIX}{ }^{\mathrm{e}}$ siècle voit donc apparaître une maîtrise de plus en plus importante de la fécondité légitime associée à une diminution du célibat.

Pour illustrer notre propos, nous avons choisi de prendre comme exemple la population du canton de Châteauponsac (Haute-Vienne). Cette population, essentiellement agricole, dominée par la petite propriété, s'est caractérisée, jusqu'à la fin du XIX siècle, par une pratique très importante de la migration saisonnière. Ce caractère ancien, puisqu'on en relève la trace depuis le XIII ${ }^{\mathrm{e}}$ siècle, a forgé la mentalité de 
nombreuses générations de paysans limousins. Cette migration était une réponse adaptative aux pressions démographiques et économiques et durait de mars à novembre. Elle conduisait une majorité d'hommes jeunes et valides issus du monde paysan vers les grands centres urbains en développement pour y exercer les métiers du bâtiment. En augmentant les ressources des familles, cette pratique migratoire accroissait la taille de la population en favorisant la multiplication des couples. Cette réduction du célibat a permis un plus grand accès des femmes à la fécondité légitime. Le pouvoir économique supérieur dont jouissaient les migrants avait une grande influence dans les stratégies matrimoniales et sur la valeur des dots. La structure sociale de cette population agricole fut duale jusqu'à la fin des années 20. A une hiérarchie sociale proprement agricole composée de petits propriétaires, de métayers, de journaliers et de domestiques agricoles, s'ajoutait la palette des métiers du bâtiment (maçons, paveurs, charpentiers, couvreurs...) répartis eux-mêmes selon une hiérarchie interne à chaque corporation puisque l'on pouvait commencer comme manœuvre et devenir chef de chantier, voire même petit entrepreneur. Les migrants se recrutaient principalement dans les familles de petits propriétaires qui étaient d'ailleurs majoritaires dans le canton.

Cette migration temporaire ne servait pas uniquement à trouver une activité économique pour la population active masculine excédentaire. En plus du pouvoir économique qu'elle procurait, elle est devenue un rite de passage à caractère social auquel on devait se plier pour jouir d'un certain prestige auprès des autres restés au village. Cette double hiérarchie permettait des ascensions sociales chez les maçons migrants, promotions qui pouvaient être plus rapides qu'en milieu rural où la transmission patrimoniale passait obligatoirement par la mort du père 
ou par l'entrée «en gendre 》 dans sa belle-famille. Ainsi, l'exploitation agricole demeurait un élément structurant de la vie sociale locale. Il est d'ailleurs possible d'avoir une bonne estimation de l'amplitude de la migration grâce aux méthodes indirectes que représentent l'analyse des variations saisonnières de mariages et de naissances. En effet, la migration temporaire laisse des traces dans les caractéristiques démographiques d'une population. Certains phénomènes, comme la nuptialité ou la fécondité, sont largement affectés par le rythme de ces déplacements. Les mariages se pratiquaient lors des retours des maçons, durant les mois de janvier, février et mars. Coutume saisonnière de mariages et mois de conceptions durant la période hivernale révèlent le mode particulier d'organisation de la société limousine.

1. Les structures familiales. - Pour l'anthropologie démographique, l'unité d'étude fondamentale est la famille. En milieu rural plus qu'ailleurs, la famille constitue une unité reposant à la fois sur des liens de parenté, de production et de consommation. La famille est un concept ancien en sciences sociales et il en est même un des éléments constitutifs, puisque ce sont les monographies familiales réalisées par des enquêtes directes, qui avaient été choisies par F. Le Play et la Société d'économie sociale comme méthode la plus appropriée plus analyser les sociétés. La famille paysanne, en particulier celle organisée en famille-souche où trois générations cohabitent, semble figée dans des attitudes invariables. Elle représentait le « modèle organisationnel parfait » (Le Play, 1871). Certaines parties du sud-ouest et du centre de la France ont conservé durablement ce type d'organisation familiale. Sans toutefois pouvoir être totalement assimilée aux communautés taisibles du centre de la France, la famille-souche limousine présente des caractéristiques différentes de celle du Sud-Ouest, 
principalement en ce qui concerne l'identification de la famille à la maison. En Limousin, l'héritier ou le gendre ne prend pas le nom de la maisonnée, comme cela peut-être le cas dans les baronnies pyrénéennes (cf. Fauve-Chamoux, 1987). Pour le reste, elle est identique et comprend un couple de parents, un des enfants avec son conjoint (l'héritier), les enfants de ceux-ci et éventuellement un frère ou une sœur de l'héritier.

Il faut bien connaître ce type d'organisation familiale pour comprendre l'organisation de la société rurale limousine, car elle influence directement l'activité économique agricole, la migration temporaire, le mode de transmission du patrimoine, le système dotal, le choix du conjoint, le nombre d'enfants...

2. Les comportements reproductifs. - La structure de la famille associée au mode de faire-valoir sont les principaux facteurs explicatifs des comportements reproductifs du monde rural.

A) L'âge au mariage. - L'âge au premier mariage est considéré comme un facteur important par les démographes dans la dynamique reproductive de la population car il marque le début de la vie féconde des couples. Les historiens démographes avaient souvent surestimé le rôle de ce facteur en pensant que le mariage tardif des femmes devait jouer un rôle régulateur essentiel dans les cas de fécondité non dirigée. A Châteauponsac, on n'observe pas de différences significatives d'âge au premier mariage entre les hommes migrants et les hommes sédentaires pour l'ensemble du $\mathrm{XIX}^{\mathrm{c}}$ siècle (25,9 ans et 26,0 ans). Par contre, on constate un allongement général de l'âge au mariage tout au long du XIX ${ }^{\mathrm{e}}$ siècle $(21,4$ ans pour les agriculteurs en 1800 et 26,1 ans en 1900). Cette tendance souligne une difficulté d'installation de plus en plus prononcée pour les hommes. Ils demeurent plus longtemps 
maçons, se marient plus tardivement et perturbent l'ensemble du marché matrimonial en freinant la circulation des dots. L'âge au premier mariage des femmes suit la même tendance que celle des hommes durant la première moitié du XIX ${ }^{\mathrm{e}}$ siècle, puis s'abaisse très rapidement jusqu'à la fin du siècle, surtout chez les épouses de migrants (22,5 ans à 20 ans).

Ce phénomène d'accroissement de l'âge au mariage pour les hommes semble assez spécifique à la région, car ce n'est pas le cas dans d'autres régions françaises. L'âge au mariage des femmes limousines, déjà considéré comme précoce au XVIII ${ }^{\mathrm{e}}$ siècle, continue de l'être au XIX ${ }^{c}$ siècle. Pour l'ensemble de la région, il se situe entre 20 et 24 ans alors qu'il varie de 21,5 à 27,5 ans pour l'ensemble de la France durant la même période. De nos jours, l'âge au mariage chez les agriculteurs de Châteauponsac s'est abaissé de deux ans entre les deux dernières générations, que ce soit pour les hommes ou pour les femmes. L'âge au premier mariage des agriculteurs de Châteauponsac continue à être précoce (21,6 ans contre 23,1 ans pour l'ensemble des agriculteurs français), phénomène que l'on peut mettre en relation avec l'accès rapide des jeunes agriculteurs limousins à la direction de l'exploitation.

B) La fécondité. - Les agricultrices n'adoptent pas de comportements féconds homogènes et des différences importantes apparaissent entre populations rurales. Ainsi, une étude comparative entre deux populations rurales vivant au Mexique et en Grèce fait-elle apparaître des profils très contrastés. Seul le tiers des femmes rurales grecques de la commune Stira dans l'île d'Elvia ont plus de 2 enfants alors que le tiers des femmes mexicaines de Chimchila dans la province du Yucata (Mexico) en ont plus de 6 (cf. fig. 9). Le travail féminin, l'éducation, la culture ou encore l'appropriation des moyens de production expliquent la variabilité de la fécondité. 
Fermmes Mayas

Femmes Grecques

$(N=102)$

$(N=91)$

Nombre

d'enfants

vivants

$N \% \quad \%$ cum. $\quad N \quad \% \quad \%$ cum.

\begin{tabular}{rrrrrrr}
0 & 3 & 2,9 & 2,9 & 3 & 3,3 & 3,3 \\
1 & 12 & 11,8 & 14,7 & 7 & 7,7 & 11,0 \\
2 & 7 & 6,9 & 21,5 & 48 & 52,7 & 63,7 \\
3 & 14 & 13,7 & 35,3 & 22 & 24,2 & 37,9 \\
4 & 15 & 14,7 & 50,0 & 6 & 6,6 & 94,5 \\
5 & 11 & 10,8 & 60,8 & 2 & 2,2 & 96,7 \\
6 & 10 & 9,8 & 70,6 & 2 & 2,2 & 98,9 \\
7 & 12 & 11,8 & 82,4 & & & 98,9 \\
8 & 11 & 10,8 & 93,2 & 1 & 1,1 & 100,0 \\
9 & 3 & 2,9 & 96,1 & & & \\
10 & 3 & 2,9 & 99 & & & \\
11 & 1 & 1,0 & 100 & & & \\
& 102 & 100 & 100 & 91 & 100 & 100,0 \\
\cline { 3 - 6 } Total & 100 & & & & & \\
\hline
\end{tabular}

Fig. 9. - Comparaison de la distribution du nombre d'enfants vivants chez des femmes Mayas et Grecques (d'après Beyene, 1989).

Dans la population agricole de Châteauponsac, le rythme des naissances chez les femmes vivant au début du XIX ${ }^{\mathrm{e}}$ siècle était assez constant tout au long de leur vie féconde et leur taux maximal de reproduction se situait dans la classe d'âge 25-29 ans. Jusqu'en 1879, la morphologie de la fécondité va conserver la même forme: le taux maximum se situe toujours dans la classe d'âge 25-29 ans, même si la valeur de celui-ci est de plus en plus basse. A partir de 1880-1899, c'est la classe 20-24 ans qui présente un taux maximal, marquant ainsi un rajeunissement de l'âge à la fécondité et un raccourcissement de la durée de vie féconde. C'est en réalité la diminution de la mortalité infantile qui permet d'atteindre plus rapidement le nombre total d'enfants souhaités. La comparaison de la variation des 
taux de fécondité entre générations et suivant les classes d'âge confirme bien les modalités de la baisse du niveau de fécondité : entre les générations 1800 et 1820 , celui-ci décroît dans toutes les classes d'âge. Entre les générations 1840 et 1860 , la fécondité chute dans les classes d'âge les plus élevées alors que dans le même temps, elle augmente dans les classes d'âge les plus jeunes. Ce phénomène va s'amplifier jusqu'à la génération 1900. Puis, à partir de cette date, la diminution de la fécondité touchera l'ensemble des femmes.

L'âge moyen à la première naissance est assez bas, il suit d'environ un an et demi en moyenne l'âge au premier mariage de la femme et demeure assez constant au cours du temps. Par contre, l'âge à la dernière naissance diminue régulièrement du début $\mathrm{du} \mathrm{XIX}^{\mathrm{e}}$ siècle à nos jours (dix ans d'écart à la dernière naissance entre 1800 et 1960) alors que le phénomène inverse s'observe dans d'autres régions. Ce résultat traduit d'une part, un contrôle très important de la fécondité et d'autre part, un désir de concentrer la période de reproduction sur les années qui suivent le mariage.

C) Les intervalles intergénésiques. - Nous avons déjà constaté au cours du temps une relative stabilité de la durée de l'intervalle proto-génésique de la population rurale de Châteauponsac. La même constatation peut être faite pour les intervalles intergénésiques. Néanmoins, une analyse plus fine laisse apparaître certaines nuances. Si l'on compare les périodes 1820,1880 et 1920 (périodes prises comme exemples et correspondant respectivement à la première moitié du XIX ${ }^{\mathrm{e}}$ siècle, à la deuxième moitié $\mathrm{du} \mathrm{XIX}{ }^{\mathrm{c}}$ siècle et à la première moitié $\mathrm{du} \mathrm{XX}^{\mathrm{e}}$ siècle), on constate pour la première période que la constitution d'une famille de 6 enfants s'effectue sur une durée de cent quatre-vingt mois soit environ cinquante mois de moins que pour les générations 1880 et 1920 qui ont besoin respectivement de deux cent trente et deux cent vingt et un mois. Les deux premières 
naissances s'effectuent sur une période assez semblable pour ces trois générations mais les intervalles suivants se raccourcissent pour les générations les plus récentes, marquant ainsi une volonté d'avoir des enfants sur un laps de temps plus rapproché.

D) La descendance finale. -- Pour qu'une population assure son autoreproduction, elle doit avoir un nombre de naissance égal de génération en génération. Mais les ressources mises à la disposition de chaque génération ne doivent pas subir de fluctuations trop importantes (le terme ressources est pris ici au sens large: alimentation, éducation, travail...). Dans le cas contraire, en cas de crise, il y aura soit diminution de la fécondité, soit émigration si le niveau de fécondité demeure constant.

A Châteauponsac, le nombre de naissances vivantes par femme ne fait que diminuer depuis le début du XIX ${ }^{\mathrm{e}}$ siècle, passant de 5,5 en 1800 à 2,9 en 1950. Cette diminution met en évidence la transformation de la société paysanne, en soulignant d'une part, l'indépendance existant entre ressources et niveau de fécondité, et d'autre part, la relation entre la diminution du nombre de grossesse et le faible taux de mortalité infanto-juvénile. La baisse de la mortalité peut se décomposer en trois phases : une première qui va de 1800 à 1839 avec un fort rythme de diminution, puis un ralentissement du rythme de décroissance entre 1840 et 1899 et enfin, à partir de 1900, la reprise d'un rythme de diminution plus rapide. Cette tendance influence le rythme de constitution des fratries. Les familles nombreuses étaient importantes au début du $\mathrm{XIX}^{\mathrm{e}}$ siècle avec une fratrie dominante à 56 enfants. Dès 1820, les familles de 3-4 enfants deviennent majoritaires. A partir de 1920, la famille de 1-2 enfants sera la plus fréquente et deviendra quasi exclusive à partir de 1960.

On remarque que la natalité est légèrement plus 
élevée dans les familles à héritier masculin dans la seconde moitié du XIX siècle. On peut avancer l'explication que les hommes sont moins absents dans ces familles que dans les familles avec une fille héritière (car dans ce cas, les maris sont deux fois plus souvent migrants et donc absents neuf mois par an durant environ dix ans de leur vie). Cette hypothèse est confirmée par la différence observée dans la descendance finale entre femmes de sédentaires et femmes de migrants. Les dernières ont une fécondité plus basse alors que la variance du nombre d'enfants est beaucoup plus régulière dans les familles-souches avec héritier.

\section{Les familles agricoles aujourd'hui. - La} démarche analytique préconisée par l'anthropologie démographique est particulièrement performante pour comprendre les relations existant entre variables démographiques et comportements familiaux. A Châteauponsac, les jeunes couples d'agriculteurs affirment leur désir d'indépendance face à une structure familiale jugée trop pesante. Le maintien (famillesouche) ou l'abandon (famille nucléaire) du mode de vie familial traditionnel correspond à des comportements démographiques spécifiques. Si l'âge au premier mariage des agriculteurs est indépendant de la structure familiale dans laquelle ils vivent, il est associé, par contre, à l'intervalle protogénésique et à la fécondité. Parmi les couples âgés (fig. 10), l'intervalle protogénésique est assez long (31,7 mois) dans les familles complexes mais il est associé à un nombre total d'enfants plus élevé que dans les familles nucléaires. Ainsi, on constate chez les couples d'agriculteurs cohabitant avec des parents capables de s'occuper de leurs petitsenfants que cette situation les encourage à avoir des conceptions moins précoces et pourtant plus nombreuses. Cependant, pour la dernière génération d'agriculteurs vivant au sein de familles complexes, l'intervalle protogénésique est devenu beaucoup plus 
court que celui des agriculteurs appartenant à des familles nucléaires.

Le nombre d'enfants du couple semble lié à la structure et à la dimension actuelle de la famille. La famille nucléaire comprend 3,7 personnes en moyenne dont 2,16 enfants et la famille-souche 5,8 personnes dont 2,45 enfants.

Type de famille

Variables démographiques

Nucléaire Multiple

Age au mariage (mari)

+45 ans

-45 ans

26,5

25,2

23,7

23,9

Age au mariage (femme)

+45 ans

-45 ans

22,5

22,1

20,5

20,7

Intervalle protogénésique (en mois)

+45 ans

-45 ans

19,6

20,0

31,7

15,9

Nombre d'enfants

+45 ans

-45 ans

3,00

2,57

2,16

2,45

Fig. 10. - Relations entre la structure familiale et le comportement démographique.

Aujourd'hui, le nombre d'enfants du couple n'est plus influencé par l'âge au mariage de l'épouse comme c'était le cas lors des siècles passés. Les femmes de 45 ans qui se sont mariées à l'âge de 20 ans n'ont pas eu plus d'enfants que celles qui se sont mariées après $25(2,6$ enfants en moyenne pour les premières et 2,7 pour les secondes). En revanche, la durée de l'intervalle protogénésique peut avoir des incidences sur la dimension de la descendance finale du couple. En général, un intervalle court, associé à un âge au 
mariage assez bas, implique un nombre d'enfants important. Chez les couples les plus jeunes, l'âge au mariage plus précoce se conjugue à un délai de conception postnuptiale plus court. Il en résulte un avancement de la première naissance de deux années par rapport à celle des couples plus âgés. La conséquence de cet abaissement de la durée de l'intervalle protogénésique se traduit par un léger accroissement du rythme des naissances chez les couples les plus jeunes : 2,48 enfants chez les femmes de moins de 45 ans en dix ans de mariage contre 2,24 enfants chez les autres. Le mode de faire-valoir peut encore influencer la dimension de la fratrie. Les petits propriétaires ont davantage d'enfants que les fermiers, ce qui n'est pas paradoxal si on sait que les potentialités de reprise de l'exploitation sont plus élevées chez les fermiers et que les enfants de petits propriétaires sont davantage contraints de quitter l'agriculture.

Les forts niveaux de corrélation observables entre le faible nombre d'enfants, la fréquence de familles peu nombreuses et une absence de mortalité infantile ne sont pas surprenantes puisqu'elles sont toutes la marque de l'apparition du modèle «moderne» de reproduction. Si la relation entre diminution du nombre d'enfants et diminution de la mortalité infantile demande à être approfondie, les autres corrélations offrent peu d'intérêt dans la mesure où elles ne font que confirmer des processus démographiques bien connus. Par contre, l'âge au premier mariage de la femme, l'espacement entre le mariage et la première naissance, ainsi que l'espacement entre la première et la seconde naissance sont à la fois indépendants entre eux et de tous les autres facteurs. Le contrôle de la fécondité était donc très performant. Il ne passait pas par les mécanismes mis en place dans les populations préindustrielles dites à fécondité « naturelle » : âge tardif au premier mariage ou maintient d'intervalles génésiques assez longs et constants 
durant la vie féconde des femmes. En fait, ce qui est remarquable, c'est que les femmes ne se sont pas mises à accroître systématiquement la durée de chaque intervalle intergénésique pour réduire leur nombre d'enfants. Tout semble indiquer qu'elles exerçaient depuis longtemps un contrôle rigoureux de leur fécondité. Elles donnaient naissances au nombre d'enfants souhaité puis stoppaient leur vie féconde. Ce contrôle de la natalité est confirmé par l'analyse de l'âge des femmes à leur dernière naissance en fonction de la parité. Il a tendance à diminuer, mais il est faible et confirme bien une ancienneté du contrôle de la fécondité. Ce qui n'empêchait pas les « accidents", c'est-à-dire ces naissances survenues tardivement comme l'atteste parfois la longueur du dernier intervalle génésique chez certaines femmes. En suivant les transformations qui affectent de petites populations, l'anthropologie démographique met en évidence à la fois le maintien et le changement de comportements entre générations. Ainsi, le monde rural n'est pas homogène dans ses attitudes, et c'est surtout la mobilité des hommes qui a marqué les populations rurales. Les historiens ont souvent pensé que le dépeuplement des campagnes françaises avait comme cause principale un exode rural ancien qui aurait attiré les éléments les plus jeunes ou les plus vigoureux vers les villes. Cet exode est bien connu pour le Limousin à partir de 1914, mais, avant cette date, la migration des hommes était de nature temporaire et constituait une solution originale d'adaptation économique et sociale aux conditions environnementales. C'est cette forme de migration qui a permis paradoxalement le maintien en place d'une population nombreuse dans une région à faibles ressources agricoles. Ce phénomène paraît général, comme le confirme, par exemple, la migration temporaire observable aujourd'hui dans certaines régions montagneuses d'Afrique. 


\section{BIBLIOGRAPHIE}

Acheson J., A cohort analysis of two generations of emigrants from northwest Portugal, in Vers un ailleurs prometteur. L'émigration, une réponse universelle à une situation de crise?. Paris et Genève, PUF/ IUED, 1993, 335-347.

Attias-Donfut C., Sociologie des générations. L'empreinte du temps, Paris, PUF, 1988.

Augustins G., Comment se perpétuer? Devenir des lignées et destins des patrimoines dans les paysanneries européennes, Nanterre, Société d'ethnologie éd, 1989,434 p.

Bahuchet S., Utilisation de l'espace forestier par les Pygmées Aka, chasseurs-cueilleurs d'Afrique centrale, Inform. sciences sociales, $1979,18,6,999-1019$.

Baker P. T., Human adaptation to high altitude, Science, 1969, 163, 1149-1156.

Bentley G. R., Golberg T., Jasienska G., The fertility of agricultural and non-agricultural traditional societies, Population studies, 1993 , 47, 269-281.

Beyene M., From menarch to menaupose, University of New-Mexico Press, 1989.

Birdsell J. B., Some environmental and cultural factors influencing the structuring of Australian Aboriginal Populations, American Naturalist, $1953,87$.

Birdsell J. B., A basic demographic unit, Current Anthropology, 1973, $14,4,337-356$.

Blurton Jones N. G., Smith L. C., O'Connell J. F., Hawkes K., Kamuzora C. L., Demography of the Hadza, an increasing and high density population of Savanna foragers, American Journal of Physical anthropology, 1992, 89, 159-181.

Bodmer W. F., Cavalli-Sforza L., Genetics, evolution and man, San Francisco, Freeman \& Company, 1968.

Bongaarts J., Potter R., Fertility, biology and behavior: an analysis of the proximate determinants, New York, Academic Press, 1983.

Bonneuil N., Jeux, équilibres et régulation des populations sous contrainte de viabilité. Une lecture de l'œuvre de l'anthropologue Fredrik Barth, Population, 1997, 4, 947-976.

Boserup E., Evolution agraire et pression demographique, Paris, Flammarion, 1970.

Bouchard G., De Braekeleer M., Population et génétique dans l'est du Québec, Québec, Presses Universitaires du Québec, 1990.

Boukir H., Croissance urbaine et problème de l'eau dans la médina de Fès (Maroc). Contribution à l'analyse de la relation population/environnement en milieu urbain, Thèse Université de Provence, 1995. 
Boyce A. J., Kuchemann C. F., Harrisson G. A., Neighbourhood knowledge and the distribution of marriage distances, Ann. Hum. Genet., 1967, 30, 335-338.

Cavalli-Sforza L., Bodmer W. F., The genetics of human populations, San Francisco, Freeman \& Company, 1971.

Cazes M-H., Chacun appartient évidemment à une génération. Mais laquelle?, Population, 1987, 4-5, 671-684.

Cazes M-H., Genetic Origins of the Dogon Population in the Arrondissement of Boni (Mali), Am. J. Fum. Genet., 1986, 39, 96-111.

Chagnon N. A., Studying the Yanomano, New York, Rinehart \& Winston, 1974.

Chapman A. M, Jacquard A., Un Isolat d'Amérique centrale: les Indiens Jicaques du Honduras, in Génétique et population. Paris, INED, 1971, 163-185.

Chesnais J-C., La transition démographique, étapes, formes, implications économiques, Paris, PUF, 1986.

Crognier E., Zarouf M., Fécondité et milieu socioéconomique dans la ville de Marrakech, Revue de la Faculté des Sciences de Marrakech. 1987, 3, 7-62.

Crow J. F., Mange A. P., Measurement of inbreeding from the frequency of marriages between persons of the same surname, Eugenics Quarterly, 1965, 12, 199-203.

Davis K., Populations and resources: facts and interpretations, in Resources, environment and population, K. Davis et M. S. Bernstam (eds), New York, Oxford University Press, 1991.

Dornstreich M. D., Morren G. E., Does New Guinea cannibalism have nutritional value?, Human Ecology, 1974, 2, 1-12.

Fargues Ph., Un siècle de transition démographique en Afrique méditérranéenne, Population, 1986, 41, 2, 205-231.

Fassin D., La production locale de la sánté publique, Prévenir, 1996, 31, 9-19.

Fauve-Chamoux M. A., Le fonctionnement de la famille-souche dans les Baronnies des Pyrénées avant 1914, Annales de démographie historique, 1987, 241-262.

Fox R., Anthropologie de la parenté, Paris, Gallimard, 1972.

Fraser S. E., Australian aborigines: a growing or contracting minority ?, Immigrants and minorities, 1986, 5, 2, 204-217.

Girard A., Le choix du conjoint. Une enquête psycho-sociologique en France, Paris, INED, 1964.

Gomila J., Définir la population, in L'étude des isolats, Paris, INED, $1977,5-36$.

Goody J., L'évolution de la famille et du mariage en Europe, Paris, A. Colin, 1985.

Hallowell A. I., The size of Algonkian hunting territories : a function of ecological adjustment, American Anthropologist, 1949, 51, 35-45.

Harvey R. G., Suter D., Migration in the Faroe Islands, Journal of Human Evolution, 1984, 13, 311-317.

Hassan F. A., Determination of the size, density and growth rate of hunting-gathering populations, in Population, ecology and social evolution, La Haye, Mouton, 1975, 27-52.

Henry L., Problèmes de la nuptialité. Considérations de méthode, Population, 1968, 5, 835-844. 
Henry L., La fécondité naturelle : observations, théorie, résultats, Population, $1961,16,625-636$.

Hilali K.. Étude biodémographique et sanitaire des populations périurbaines de la zone d'El Azzonzia (Maroc), thèse de l'Université de Marrakech, 1986.

Hill K., Hawkes K., Hurtado M., Kaplan H., Seasonal variance in the diet of Ache Hunter-Gatherers in Eastern Paraguay, Human Eco$\log y, 1984,12,101-136$.

Howell N., Demography of the Dobe Kung, London, Academic Press, $197 \%$.

Howell N., Demographic anthropology, Anmual Review of Anthropology, 1986, 15, 219-246.

Imaizumi Y., Factors influencing the frequency of consanguineous marriages in Japan : marital distance and opportunity of encounter, Human Heredity; 1986, 36, 304-309.

Jacquard A. (sous la dir. de), L'étude des isolats. Espoirs et limites, Paris. INED, 1977.

Jacquard A., Structures génétiques des populations, Paris, Masson, 1970.

Jaffe K., Chagon-Puignea G., Assortative mating: Sex differences in mate selection for married and unmarried couples, Human Biology, $1995,67,1,111-120$.

Kucheman C. F., Boyce A. J., Harrison G. A., A demographic and genetic study of a group of Oxfordshire villages, Human Biology, $1967,3,251-276$.

La Recherche, Dossier: Comment évolue la population française, $L a$ Recherche, 1990, 224, 1106-1115.

Laburthe-Tolra P., Warnier J.-P., Ethnologie, anthropologie, Paris, PUF, 1993.

Lasker G. W., Chiarelli B., Fedele F., Kaplan, B. A., Degree of human genetic isolation by isonymy and marital distances in two communities in an Italian Alpine valley, Human Biology, 1972, 44, 351-360.

Laslett P., La famille et le ménage: approches historiques, Annales ESC, 1972, 4-5, 847-872.

Laughlin W. S., Aleutian ecosystem, Science, 1970, 169, 1107-1108.

Lee R. B., Kung bushman subsistance; an input-output analysis, in A. P. Vayda (Ed.), Environment and cultural behavior, University of Texas, $1969,47-79$.

Lee R. B., Devore I. (Eds), Man the Hunter., Chicago, Aldine, 1968.

Lelièvre E., Bonvalet C., Bry X., Analyse biographique des groupes. Les avancées d'une recherche en cours, Population, 1997, 4, 803-830.

Lévi-Strauss C., Les structures élémentaires de la parenté, La Haye, Mouton, 1949.

Lewontin R., La diversité des hommes; l'inné, l'acquis et la génétique, Paris, Belin, 1982.

Mair L., Le mariage, Paris, Payot, 1974.

Mc Elroy A., Townsend P. K., Medical Anthropology in Ecological perspective, Boulder, Westvien Press, 1989.

Mead M., Le fossé des générations, Paris, Denoël/Gonthier, 1971.

Neel J. V., Chagnon N. A., The demography of two tribes of primitive relatively unacculturated american indians, Proceedings of the National Academy of Sciences, 1968, 59, 3, 680-689. 
Pascon P. Population et développement. Éléments de psychologie d'une démographie volontaire au Maroc, Bulletin économique et social du Maroc, 1986, 6, 195-209.

Peatrik A-M., Ages de la vie, temps de la société, in Le temps et la démographie, Louvain-la-Neuve, Academia/L'harmattan, 1994, 127142.

Penrose L. S., Natural selection in Man. Some basic problems, in Natural selection in human populations, Pergamon Press, 1959, 1-11.

Pison G., Hill K., Cohen B., Foote K., Les changements démographiques au Sénégal, Paris, PUF/INED, 1997.

Pison G., La démographie de la polygamie, La recherche, 1985, 16, 168, 894-901.

Pressat R., Dictionnaire de démographie, Paris, PUF, 1982.

Robert-Lamblin J., Famille biologique et famille sociale à Ammassalik côte est du Groenland, Etudes Inuit, 1978, 2, 2, 23-35.

Roberts D. F., Les concepts d'isolats, in L'étude des isolats. Espoirs et limites, Paris, INED, 1977, 75-92.

Ruffié J., De la biologie à la culture, Paris, Flammarion, 1976.

Sahlins, M., Age de pierre, age d'abondance, Paris, Gallimard, 1976.

Sauvain-Dugerdil C., Boetsch G., Serrano-Sanchez C., Continuity, collapse or metamorphosis? Demographic anthropology and the study of change within human populations, International Journal of Anthropology, 1996, 2/4, 3-11.

Segalen M., Jacquard A., Choix du conjoint et homogamie, Population, $1971,3,487-498$.

Segalen M., Sociologie de la famille, Paris, A. Colin, 1981.

Singly $\mathrm{F}$. de, Théorie critique de l'homogamie, L'annee sociologique, $1987,37,182-205$.

Sutter J., Tabah L., Stiucture démographique et génétique de l'isolat des Esquimaux polaires (Thulé, Groenland). Acta Genet., 1957, 6, 391-395.

Tabutin D., Gourbin C., Mortalité et santé des enfants en Afrique du Nord depuis les années soixante, une synthèse comparative, Cahiers de l'IREMAM, 1997, 9/10, 9-30.

Tapinos G., Eléments de démographie, Paris, A. Colin, 1985.

Tindale M. B., Tribal and intertribal marriage among the Australian aborigines, Human Biology, 1953, 25, 3, 169-190.

Townsend P. K., New Guinea sago gatherers-A study of demography in relation to subsistence, in Food, ecology and culture, New York, Gordon \& Breach, 1980, 21-26.

Van de Walle E., L’âge au mariage: tendances récentes, in Changements démographiques en Afrique subsaharienne, Paris, PUF/INED, $1996,119-154$

Van Der Kroef J. M., Some head-hunting traditions of southern NewGuinea, American Anthropologist, 1952, 54, 221-235.

Walhund S., Zusammensetzung von populationen und Korrelationserscheinungen vom Standpunkt der Vererbungslehre aus betrachtet, Hereditas, 1928, 11, 65-106.

Wright $\mathrm{S}$., Isolation by distance under diverse systems of mating, Genetics, 1946, 31, 39-59.

Wrigley E. A., Population and history, New York, MacGraw-Hill, 1976.

Yasuda N., The distribution of distance between birthplaces of mates, Human Biology, 1975, 47, 1, 81-100. 


\section{TABLE DES MATIÈRES}

Introduction

Chapitre I - Concepts et méthodes

I. Les concepts anthropologiques, 6:1. L'évolution, l'adaptation, $7 ; 2$. Le concept de population, 8; 3. Alliance et parenté, 16 - II. Les théories et méthodes démographiques, $24: 1$. Connaître les théories, 24 ; 2. Utiliser les méthodes, 30 -- III. Les estimateurs de la structure génétique et de l'évolution biologique, $34: 1$. Le coefficient de consanguinité d'un individu, $35 ; 2$. Le coefficient de consanguinité d'une population, 36 ; 3. La probabilité d'origine des gènes, 39.

\section{Chapitre II - L'anthropologie démographique}

I. Le champ de l'anthropologie démographique, 41 : 1. La famille, $41 ; 2$. La reproduction, $42 ; 3$. Le concept de génération, 44 - II. Les méthodes utilisées dans les recherches d'anthropologie démographique, $48: 1$. La notion d'observatoire et le fichier de population, $49 ; 2$. L'état civil et la méthode généalogique, 51 ; 3. La liste nominative de recensement et la structure des ménages, $53 ; 4$. L'enquête biodémographique dans les pays en développement, $55 ; 5$. Échelle d'étude et homogénéité de la population, $57 ; 6$. L'apport des méthodes qualitatives, 58 - III. Les principales analyses en anthropologie démographique, $59: 1$. Structures familiales, formation et composition du ménage, $59 ; 2$. La mortalité, $61 ; 3$. La fécondité, $64 ; 4$. La migration, 67.

\section{Chapitre III - Quelques exemples}

I. Les populations de chasseurs-cueilleurs, 73: 1. Environnement et population, $73 ; 2$. Les paramètres démographiques dans les populations de chasseurs-cueilleurs, 82 - II. Les populations rurales des pays en développement : l'exemple africain, $96: 1$. Les déterminants de la nuptialité, 97;2. La gestion de la fécondité, 102; 3. La mortalité infantile et le rôle du milieu, 107 - III. Les populations rurales occidentales: l'exemple de Châteauponsac, 109:1. Les structures familiales, 112; 2 . Les comportements reproductifs, $113 ; 3$. Les familles agricoles aujourd'hui, 118.

Bibliographie 


\title{
BIBLIOGRAPHIE THÉMATIQUE «QUE SAIS-JE?»
}

\author{
La dénographie, $\mathrm{n}^{\circ} 2546$ \\ Population et développement, n' 2842 \\ Géographie de la population, $\mathrm{n}^{\mathrm{a}} 1187$ \\ Les migrations, $\mathrm{n}^{\circ} 224$ \\ L'écologie humaine, $\mathrm{n}^{\circ} 1607$
}




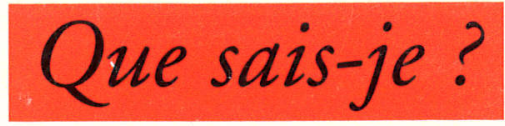

\section{COLLECTION ENCYCLOPÉDIQUE}

fondée par Paul Angoulvent

dirigée par Anne-Laure Angoulvent-Michel

L'anthropologie démographique a pour objet l'étude des processus de reproduction et d'évolution des petits groupes humains en utilisant les concepts et les méthodes de l'anthropologie, de la démographie et de la biologie des populations. Elle étudie notamment les relations existant entre parenté, structures familiales, économie, reproduction sociale et évolution du pool génique, permettant ainsi d'apprécier les changements continuels qui affectent les populations humaines.

Daniel Bley, chargé de recherches au CNRS, est chargé de cours à l'IEP d'Aix et à la Faculté de médecine de Marseille.

Gilles Boëtsch, directeur de recherches au CNRS, est chargé de cours à l'Université d'Aix-Marseille III et à la Faculté de médecine de Marseille. II est professeur associé à l'Université de Turin.

\section{Derniers titres parus}

259 Les matériaux énergétiques
R. AMIABLE

643 Le yoga

P. FEUGA et T. Michä̈L

3340 L'Andorre
A. Degage et A. Duro i Arajol

3353 Les théories de l'art
A. Cauquelin

3355 La science régionale

G. BENKO

3358 L'évaluation des politiques publiques de l'emploi
G. Benhayoun et Y. LazzerI

3360 Les sociétés de bourse P.-J. LeHManN

3362 L'exploration spéléologique et ses techniques
E. GILLI

3364 Le conservatisme américain N. KESSLER

3366 L'hypocondrie

B. BRUSSET

3368 Chronologie de la France médiévale

T. CHARMASSON

3385 Histoire des banques centrales N. OLSZAK

3408 La pauvreté

C.-F. BARRAT 